\title{
Spin-polarized Quasiparticle Transport in Cuprate Superconductors
}

\author{
Thesis by \\ Chu-Chen $\mathrm{Fu}$ \\ In Partial Fulfillment of the Requirements \\ for the Degree of \\ Doctor of Philosophy
}

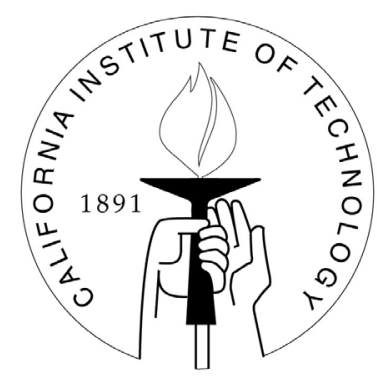

California Institute of Technology

Pasadena, California

2003

(Defended October 17, 2002) 
(C) 2003

\section{Chu-Chen $\mathrm{Fu}$}

All Rights Reserved 


\section{Acknowledgement}

There are many people who have helped me throughout the years in my graduate studies. First and foremost, I would like to thank my thesis advisor, Prof. Nai-Chang Yeh, whose guidance was instrumental in my research experiments and preparation of this thesis. Without her encouragement and patience during the rough periods in my life, I would not have been able to complete this work. I admire her dedication and work ethic, as well as her ability as a physicist to navigate through the maze in which we often find ourselves while doing research. Second, I would like to thank Dr. Richard Vasquez for supplying our group with seemingly infinite number of samples (and all those sample transport jars), as well as for giving me the opportunity (along with my advisor) to learn new techniques, to meet new people, and to expand my horizon working at JPL. I would also like to thank the members of my committee, Prof. Tombrello, Prof. Cross, and Prof. Zmuidzinas, for their time out of busy schedules.

There are many people in Prof. Yeh's group with whom I have had the privilege to work over the years. Our engineer, Nils Asplund, has worked tirelessly on constructing, assembling, upgrading, and updating our equipment. I thank him for working on and building the measure probes I have used in this work. I thank David Beam, Wen Jiang, and Dan Reed for the helping me get my feet wet when I started in the group. I also thank my fellow graduate students ChingTzu Chen and Ted Corcovilos for their assistance around the lab and for their friendship. For the many undergraduate students that I have worked with, including Hoa Huynh, Geoff Beach, Kamran Vakili, Zhao Huang, and Benjamin Gudlewski, I hope I have helped them grow as much as I have learned from them. In particular, I would like to thank Hoa, Geoff, and Zhao for their assistance in my research, enduring many sleepless nights in the lab with me. In addition, I

would like to acknowledge the postdocs I have interacted with, Dr. Arkasha Samoilov, Prof. John 
Wei, and Dr. Pierre Seneor, for their valuable discussions. In particular, I thank Pierre for his technical assistance with the lab computer software.

I am grateful to Prof. Scherer for allowing me open access to his facilities in Sloan for my device fabrication. I also received much help from his students, Marko Loncar and David Barsic. My special thanks to Dr. Yves Lassailly for always being there and for extending his warmth and friendship. I thank Prof. Dougherty for making the SQUID magnetometer available, and Jeff Clites and Frank Monzon for assisting me in the training process. I thank Ali Ghaffari for overseeing my nearly seamless transition to the equipment in the Micro/Nano cleanroom here at Caltech. I would also like to thank Bruce Bumble of JPL for his timely assistance and helpful discussions, often when I was at my most frustrated.

I am eternally indebted to my parents for the sacrifices that they have made in their own lives for us and our education. It is a sentiment that I will forever carry with me. I would like to thank my family and friends for the support and well wishes. Thank you for all your prayers. Finally, I am thankful to Wendy Chen for being there for and with me. Her support and faith have and will continue to be my inspiration. 


\begin{abstract}
The effects of spin-polarized quasiparticle transport in superconducting $\mathrm{YBa}_{2} \mathrm{Cu}_{3} \mathrm{O}_{7-\delta}$ (YBCO) epitaxial films are investigated by means of current injection into perovskite ferromagnet-insulator-superconductor (F-I-S) heterostructures. Transport and magnetic properties of these CMR perovskites are first investigated by inducing lattice distortions using lattice mismatching substrates. The half-metallic nature of these perovskites provides an epitaxially grown heterostructure, ideal for injection of spin-polarized current. These effects are compared with the injection of simple quasiparticles into control samples of perovskite nonmagnetic metal-insulator-superconductor (N-I-S). Systematic studies of the critical current density $\left(J_{c}\right)$ as a function of the injection current density $\left(J_{i n j}\right)$, temperature $(T)$, and the thickness (d) of the superconductor demonstrate the "self-injection effect" and reveal dramatic differences between the F-I-S and N-I-S heterostructures, with strong suppression of $J_{c}$ and a rapidly increasing characteristic transport length near the superconducting transition temperature $T_{c}$ only in the F-I-S samples. The temperature dependence of the efficiency $\left(\eta=\Delta J_{c} / J_{i n j} ; \quad J_{c}\right.$ : the suppression of critical current due to finite $J_{i n j}$ ) in the F-I-S samples is also in sharp contrast to that in the N-I-S samples, suggesting significant redistribution of quasiparticles in F-I-S due to the longer lifetime of spin-polarized quasiparticles. Application of conventional theory for nonequilibrium superconductivity to these data further reveals that a substantial chemical potential shift $\mu^{*}$ in F-I-S samples must be invoked to account for the experimental observation, whereas no discernible chemical potential shift exists in the N-I-S samples, suggesting strong effects of spin-polarized quasiparticles on cuprate superconductivity. The characteristic times estimated from our studies are suggestive of anisotropic spin relaxation processes, possibly with spin-orbit interaction dominating the c-axis spin transport and exchange interaction prevailing within the $\mathrm{CuO}_{2}$ planes.
\end{abstract}




\section{Publications}

1. "Spin-polarized quasiparticle transport in cuprate superconductors," C.-C. Fu, Z. Huang, and N.-C. Yeh, Phys. Rev. B 65, 224516 (2002).

2. "Investigating the pairing state of cuprate superconductors via quasiparticle tunneling and spin injection," N.-C. Yeh, C.-T. Chen, C.-C. Fu, P. Seneor, Z. Huang, C. U. Jung, J. Y.Kim, Min-Seok Park, Heon-Jung Kim, S.-I. Lee, K. Yoshida, S. Tajima, G. Hammerl, and J.Mannhart, Physica C 367, 174 (2002).

3. "Pairing symmetry and spin-polarized quasiparticle transport in high-temperature superconducting cuprates," N.-C. Yeh, C.-C. Fu, C.-T. Chen, Z. Huang, R. P. Vasquez, and S. Tajima, invited paper for the SPIE conference on "Superconducting and Related Oxides: Physics and Nano-engineering IV", Orlando, FL, April 24 - 28, 2000, Proceedings of SPIE 4058, 60 (2000).

4. "Dynamic pair breaking in cuprate superconductors via injection of spin-polarized quasiparticles in perovskite F-I-S heterostructures," N.-C. Yeh, J. Y. T. Wei, C.-C. Fu, and R. P. Vasquez, Physica B 284, 507 (2000).

5. "Magnetic percolation effect on the spontaneous Hall resistivity and magnetoresistance of $\mathrm{La}_{1-\mathrm{x}} \mathrm{A}_{\mathrm{x}} \mathrm{CoO}_{3}(\mathrm{~A}=\mathrm{Ca}, \mathrm{Sr} ; 0.1<\mathrm{x}<0.5)$," N.-C. Yeh, C.-C. Fu, R. P. Vasquez, and A. V. Samoilov, Physica B 284, 1446 (2000).

6. "Nonequilibrium superconductivity under spin-polarized quasiparticle currents in perovskite ferromagnet-insulator-superconductor (F-I-S) heterostructures," N.-C. Yeh, R. P. Vasquez, C.-C. Fu, A. V. Samoilov, Y. Li, and K. Valiki, Phys. Rev. B 60, 10522 (1999).

7. "Giant spontaneous Hall effect and magnetoresistance in $\mathrm{La}_{1-\mathrm{x}} \mathrm{Ca}_{\mathrm{x}} \mathrm{CoO}_{3}(0.1<\mathrm{x}<0.5)$," A. V. Samoilov, G. Beach, C.-C. Fu, N.-C. Yeh, and R. P. Vasquez, J. Appl. Phys. 83, 6998 (1998).

8. "Magnetic percolation and giant spontaneous Hall effect in $\mathrm{La}_{1-\mathrm{x}} \mathrm{Ca}_{\mathrm{x}} \mathrm{CoO}_{3}(0.2<\mathrm{x}<$ 0.5)," A. V. Samoilov, G. Beach, C.-C. Fu, N.-C. Yeh, and R. P. Vasquez, Phys. Rev. B 57, Rapid Communications, R14032 (1998).

9. "Intrinsic and disorder-induced anisotropic vortex dynamics of high-temperature superconductors," N.-C. Yeh, W. Jiang, C.-C. Fu, M. Konczykowski, and F. Holtzberg, Physica C 282-287, 1943 (1997).

10. "Investigation of the current-induced nonlinear vortex dynamics in $\mathrm{YBa}_{2} \mathrm{Cu}_{3} \mathrm{O}_{7}$ single crystals using transport and transmittivity measurements," W. Jiang, N.-C. Yeh, C.-C. Fu, M. Konczykowski, and P. Holtzberg, Physica C 282-287, 1947 (1997).

11. "Effects of lattice distortion on the physical properties and surface morphology of magnetoresistive perovskite epitaxial films," N.-C. Yeh, C.-C. Fu, J. Y. T. Wei, R. P. Vasquez, J. Huynh, S. M. Maurer, D. A. Beam, and G. Beach, J. Appl. Phys. 81, 5499 (1997).

12. "Effects of lattice distortion and Jahn-Teller coupling on the magnetoresistance of $\mathrm{La} 0.7 \mathrm{Ca} 0.3 \mathrm{MnO} 3$ and $\mathrm{La} 0.5 \mathrm{Ca} 0.5 \mathrm{CoO} 3$ epitaxial films," N.-C. Yeh, R. P. Vasquez, D. A. Beam, C.-C. Fu, J. Huynh, and G. Beach, J. Phys.: Condens. Matter 9, 3713 (1997).

13. "Effects of lattice distortion, polaron conduction and double-exchange interaction on the physical properties of magnetoresistive manganites and cobaltites," N.-C. Yeh, R. P. Vasquez, J. Y. T. Wei, C.-C. Fu, G. Beach, J. Huynh, A. V. Samoilov, A. V. Boris, N. N. Kovaleva, and A. V. Bazhenov, Epitaxial Oxide Thin Films- III, invited paper, Mat. Res. Soc. Proc. (1997). 


\section{Contents}

List of Figures $\quad$ x

1 Introduction $\quad 1$

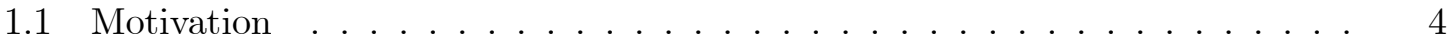

1.2 Thesis Overview . . . . . . . . . . . . . . . . . . . . 11

2 Superconductivity Background $\quad 13$

2.1 Critical Magnetic Field and Critical Current . . . . . . . . . . . . . . 13

2.2 Nonequilibrium Superconductivity . . . . . . . . . . . . . . . . 16

2.2.1 Quasiparticle Disequilibrium _. . . . . . . . . . . . 17

2.2.2 Relaxation Times . . . . . . . . . . . . . . . . . . . 20

2.2.3 Steady-State Energy-Mode . . . . . . . . . . . . . . . . 20

2.2.4 Steady-State Charge-Mode . . . . . . . . . . . . . . 22

2.3 Summary . . . . . . . . . . . . . . . . . . . . . 24

3 General Experimental Procedures 25

3.1 Sample Preparation . . . . . . . . . . . . . . . . . . 26

3.2 Cryogenic Dewars . . . . . . . . . . . . . . . . . . 28

3.2.1 Superconducting Magnet Dewar . . . . . . . . . . . . . . 29

3.2 .2 Low-Field Magnet Dewar . . . . . . . . . . . . . . . . . . . . . . . . . . . . . . 31

3.2 .3 Liquid Helium Dewar . . . . . . . . . . . . . . . . . . . . 33

3.3 The Sample Probes . . . . . . . . . . . . . . . . . . . . . 33

3.3.1 Standard Transport Probe . . . . . . . . . . . . . . . 35

3.3.2 "Quick-Dip" Probe . . . . . . . . . . . . . . . . . . . . 37

4 CMR Material and DC Transport $\quad 39$

4.1 Background . . . . . . . . . . . . . . . . . . . 40

4.2 Material and Substrate Selection . . . . . . . . . . . . . . 42

4.3 Sample Growth and Characterization . . . . . . . . . . . . . . . . . 43

4.4 CMR Sample Preparation . . . . . . . . . . . . . . . . . . . . 44

4.5 Transport Resistivity Measurement . . . . . . . . . . . . . . . . 45

4.6 Magnetization and Susceptibility Measurement . . . . . . . . . . . 46 
4.7 Results . . . . . . . . . . . . . . . . . . . . . . . 48

4.8 Analyses . . . . . . . . . . . . . . . . . . . 54

5 Spin-injection Experiment $\quad \mathbf{5 9}$

5.1 Sample Characterization . . . . . . . . . . . . . . . . . . 59

5.2 Prevention of Artifacts from Joule Heating . . . . . . . . . . . . . . . 62

5.3 Pulse-Current Technique . . . . . . . . . . . . . . . . . . . . . 64

5.4 Critical Current Suppression _. . . . . . . . . . . . . . 67

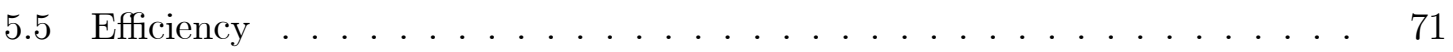

6 Analyses of Spin-Injection Results $\quad 80$

6.1 Spin-relaxation Length Derivation _ . . . . . . . . . . . . . 80

6.2 Understanding Efficiency . . . . . . . . . . . . . . . . . . . . . 82

6.3 Discussion . . . . . . . . . . . . . . . . . . . . . . . 93

6.4 Alternative Scenarios . . . . . . . . . . . . . . . . . . . . 94

6.4.1 Paramagnetic Effect . . . . . . . . . . . . . . . . 94

6.4.2 "Current Summation" . . . . . . . . . . . . . . . 95

7 Basics in Thin-Film Processes 102

7.1 Evaporation Processes . . . . . . . . . . . . . . . . . . . . . 102

7.1.1 Theory and Mechanisms . . . . . . . . . . . . . . 103

7.1 .2 Evaporation Apparatus . . . . . . . . . . . . . . . . . 105

7.2 Sputter Deposition Processes _. . . . . . . . . . . . . . . . 109

7.2 .1 Processing Plasma . . . . . . . . . . . . . . . . . . . 110

7.2.2 Plasmas in the Presence of $\mathbf{E}$ and B Fields . . . . . . . . . . . . . . 112

7.2.3 Circular Planar Magnetrons . . . . . . . . . . . . . . . . . . . 113

7.2 .4 Sputtering Target . . . . . . . . . . . . . . 115

7.2 .5 The Pump . . . . . . . . . . . . . . . . 116

7.2 .6 Sputtering Yield . . . . . . . . . . . . . . . . 116

7.3 Ion Beam Etching . . . . . . . . . . . . . . . . . . 117

7.3 .1 Ion Generation . . . . . . . . . . . . . . . . 117

7.3 .2 Ion Acceleration . . . . . . . . . . . . . . . . . . . . 119

7.3 .3 Beam Neutralization . . . . . . . . . . . . . . . 120

7.3.4 Process Control . . . . . . . . . . . . . . . . . 120

7.4 Reactive Ion Etching . . . . . . . . . . . . . . . . . . . . 121

8 Microfabrication/UV Lithography $\quad 123$

8.1 UV Lithography . . . . . . . . . . . . . . . . . . . . . . 124

8.1.1 Sample Preparation . . . . . . . . . . . . . . . . . . 124

8.1 .2 Application of Resist . . . . . . . . . . . . . . . . . . . . 124

8.1 .3 Softbake . . . . . . . . . . . . . . . . . 125

8.1.4 Photomask and Exposure . . . . . . . . . . . . . 125

8.1.5 Development and Rinse . . . . . . . . . . . . . . . . 127

8.1.6 Postbake and Photoresist Removal . . . . . . . . . . . . . . . . . . 127

8.2 Device Microfabrication . . . . . . . . . . . . . . . . . . 128 
8.3 DC Technique . . . . . . . . . . . . . . . . . . . . 135

8.4 Interface Resistance and Transfer Length . . . . . . . . . . . . . . 136

8.5 Results . . . . . . . . . . . . . . . . . . . . 138

8.6 Summary . . . . . . . . . . . . . . . . . . . 145

9 Conclusion $\quad 148$

9.1 Summary . . . . . . . . . . . . . . . . . . . . . . . 148

9.2 Possible Future Investigations . . . . . . . . . . . . . . . . 150

$\begin{array}{ll}\text { Bibliography } & 152\end{array}$ 


\section{List of Figures}

1.1 Crystalline structure of $\mathrm{YBa}_{2} \mathrm{Cu}_{3} \mathrm{O}_{7-\delta}$ high-temperature superconductor . .

1.2 Generic temperature $(T)$ vs. doping level $(\delta)$ phase diagrams for $n$-type and p-type superconductors . . . . . . . . . . . . . . . 3

1.3 Schematic representations of $s$-wave and $d_{x^{2}-y^{2} \text {-wave pairing symmetries. }}$. 6

1.4 Effects of quantum impurities on $p$-type cuprate superconductors . . . . . 7

1.5 Schematic drawing of current injection in a cross-sectional view of an F-I-S

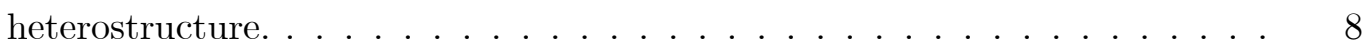

2.1 Schematics of even and odd mode disequilibrium . . . . . . . . . . . . . 19

3.1 Superconducting magnet dewar and the enlarged view of the sample space, vacuum jacket, and magnet solenoid. . . . . . . . . . . . . . 30

3.2 Low-field magnet dewar and the enlarged view of sample space. . . . . . . . 32

3.3 Top section of sample probes. . . . . . . . . . . . . . . . 35

3.4 Sample stage for standard transport probe. . . . . . . . . . . . . 36

4.1 Unit cell structure of perovskite $\mathrm{ABX}_{3} \ldots \ldots \ldots \ldots$. . . . . . . . . 40

4.2 The alignment of domains in the direction of the external magnetic field $H . \quad 41$

4.3 Magnetization and ac magnetic susceptibility vs. temperature. . . . . . . 47

4.4 The effect of lattice distortion on the resistivity of $\mathrm{La}_{0.7} \mathrm{Ca}_{0.3} \mathrm{MnO}_{3}$ epitaxial films on different substrates. . . . . . . . . . . . . 51

4.5 The magnetic field dependence of the resistivity at various constant temper-

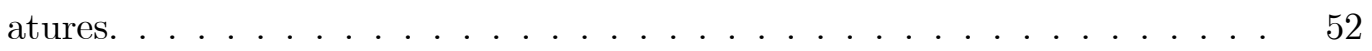

4.6 The effect of lattice distortion on the resistivity of $\mathrm{La}_{0.5} \mathrm{Ca}_{0.5} \mathrm{CoO}_{3}$ epitaxial films on different substrates. . . . . . . . . . . . . 53

4.7 The $G(T)-T$ and $G^{\prime}(H)-H$ curves for LCMO/LAO, LCMO/STO, and LCMO/YAO films. . . . . . . . . . . . . . . 56

5.1 As-grown heterostructure with gold contacts deposited. . . . . . . . . . . 60

5.2 The $R$ vs. $T$ curves of various F-I-S and N-I-S heterostructures with 100

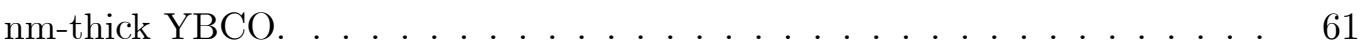

5.3 Comparison of the normalized critical current density $J_{c} / J_{c 0}$ vs. $I_{i n j} \ldots \ldots$. 63

5.4 Block diagram of the pulse-current technique. . . . . . . . . . . . . 64 
5.5 Full schematic of the pulse-current measurement including the cryostat. . . 65

5.6 Representative I-V characteristic curves for F-I-S and N-I-S samples. . . . . 73

5.7 More representative I-V characteristics of F-I-S samples. . . . . . . . . . . . 74

5.8 Comparison of the zero-field critical current density $\left(J_{c}\right)$ of various $100 \mathrm{~nm}$ thick $\mathrm{YBCO}$ and perovskite heterostructures as a function of the reduced temperature $\left(T / T_{c}\right) \ldots \ldots \ldots \ldots \ldots \ldots$

5.9 Critical current vs. injection current plot for $100 \mathrm{~nm}$-thick YBCO het-

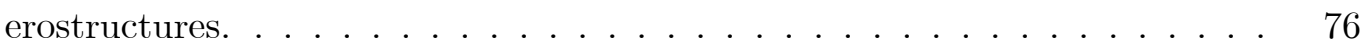

5.10 Schematic illustration of "self-injection" and external injection. . . . . . . . 77

$5.11 J_{c^{-} \text {vs.- }} J_{i n j}$ isotherms of F-I-S samples with $40 \mathrm{~nm}$ - and $160 \mathrm{~nm}$-thick YBCO. 78

5.12 Efficiency $\eta_{s}$-vs.- $J_{i n j}$ isotherms of F-I-S and N-I-S samples. . . . . . . . . 79

6.1 Characteristic $c$-axis spin relaxation length $\delta_{s}^{c}$ as a function of reduced temperature $\left(T / T_{c}\right)$ for the F-I-S heterostructures. . . . . . . . . 81

6.2 Functional form of $c_{1}(T)$ used in the simulation of $\eta_{s} \ldots \ldots \ldots$. . . . . 89

6.3 Hypothetical current flow patterns in the YBCO layer of the F-I-S heterostructure under external current injection from the CMR film. . . . . . . 96

6.4 Scanning tunneling spectroscopic (STS) studies of the YBCO layer in both F-I-S and N-I-S heterostructures. . . . . . . . . . . . . . . . . . . 98

7.1 Resistance-heated sources. . . . . . . . . . . . . . . . . . 107

7.2 General schematic of a sputtering system. . . . . . . . . . . . . . . . 110

7.3 The motion of an electron in the presence of perpendicular electric and magnetic fields. . . . . . . . . . . . . . . . . . . 113

7.4 The shape of the magnetic field of a circular magnetron cathode and the resulting plasma drift path. . . . . . . . . . . . . . . 114

7.5 A cross section of a planar magnetron sputter source. . . . . . . . . . . 115

7.6 Schematic diagram of a gridded ion source. . . . . . . . . . . . . . 118

8.1 First stage in the microfabrication to obtain the large CMR mesa. . . . . . 129

8.2 Second stage in the microfabrication to obtain the YBCO bridges. . . . . . 130

8.3 Third stage in the microfabrication to obtain the Au contact pads and to add insulator to prevent shorting in the devices. . . . . . . . . . . . . 131

8.4 Final stage in the microfabrication to obtain the contact wires. . . . . . . . 132

8.5 Injection devices, located near the center of the die pattern. . . . . . . . . 133

8.6 Close-up view of the F-I-S devices. . . . . . . . . . . . . . . . . . 134

8.7 Schematic for transfer length measurement. . . . . . . . . . . . . . . 137

$8.8 R$-vs.-T curves for CMR materials. . . . . . . . . . . . . . . . . 139

$8.9 \rho$-vs. $T$ curves for YBCO in the F-I-S devices of various bridge widths. . . . 139

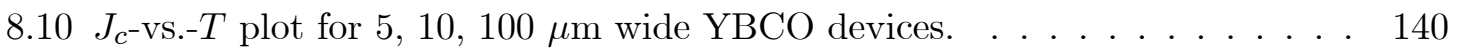

$8.11 \rho$-vs.- $T$ curves for YBCO in the N-I-S devices of various bridge widths. . . . 141

$8.12 \rho$-vs.- $T$ curves for $100-\mu \mathrm{m}$ width YBCO bridge in both F-I-S and N-I-S devices. 142

8.13 Normalized $J_{c}$ vs. $I_{i n j}$ comparison for F-I-S and N-I-S devices. . . . . . . . 143

8.14 Efficiency data for F-I-S and N-I-S devices. . . . . . . . . . . . . . . . . 144 
8.15 Normalized $J_{c}$ data from two patterned devices compared with the hypothet-

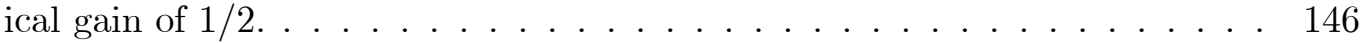

9.1 Other possible geometries for future heterostructure devices. . . . . . . . . . 151 


\section{Chapter 1}

\section{Introduction}

After superconductivity was first discovered in 1911 by H. Kamerlingh Onnes, a fundamental understanding of the phenomenon eluded physicists for many decades. It was not until in the 1950s and 1960s that a complete and satisfactory theoretical picture of the conventional superconductor emerged, with the Ginzburg-Landau (GL) theory [1] providing macroscopic and phenomenological understanding and the BCS theory [2] for rigorous, microscopic explanation. The subject of superconductivity was revitalized in 1986, after a new class of high-temperature superconductors was discovered by Bednorz and Müller [3]. These new superconductors are known as perovskite cuprates with layers of $\mathrm{CuO}_{2}$ planes stabilized by intermediate charge reservoirs (see Fig. 1.1). Although these newly discovered superconductors appear to follow some of the general phenomenology as conventional superconductors, many new phenomena are unique to the cuprates, and the basic microscopic mechanism still remains an open question to this day [4].

It is commonly believed that the characteristics of the mechanism in high-temperature 


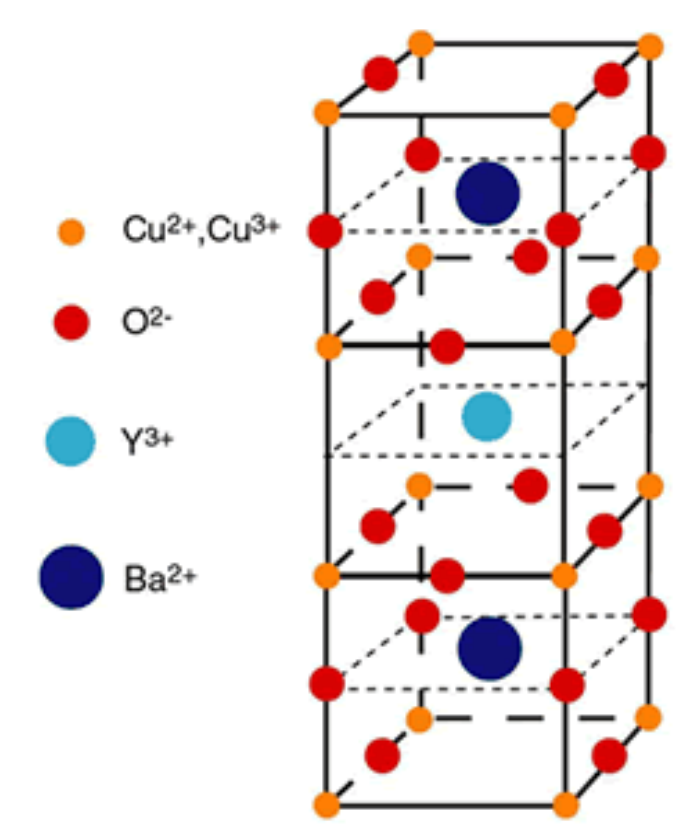

Figure 1.1: The crystalline structure of $\mathrm{YBa}_{2} \mathrm{Cu}_{3} \mathrm{O}_{7-\delta}$ (YBCO) high-temperature superconductor, chosen for this study, has the same ordered arrangement of slabs parallel to each other and stacked along the $c$-axis, like other cuprate oxides. These general features arising from the layered structure involve the alternation between conduction and binding slabs. The conduction slabs consist of copper oxide $\left(\mathrm{CuO}_{2}\right)$ layers, with each copper ion $\mathrm{Cu}^{2+}$ surrounded by four oxygen ions $\mathrm{O}^{2-}$, and each oxygen bonded to two coppers. The conduction slabs have two $\mathrm{CuO}_{2}$ layers, held in place within the slab by yttrium cations $\mathrm{Y}^{3+}$. This is depicted at the middle of the structure. The binding slabs consist mainly of layers of metal oxides, and in the case of $\mathrm{YBCO}$, there are two $\mathrm{BaO}$ layers, separated by a single layer of $\mathrm{OCu}$ layer. The conduction slabs are where the supercurrent flows, and the binding slabs hold together the conduction slabs. The other function of these binding slabs is that they are charge reservoirs, since they contain the source of charge that brings about the hole doping of the conduction layers. 


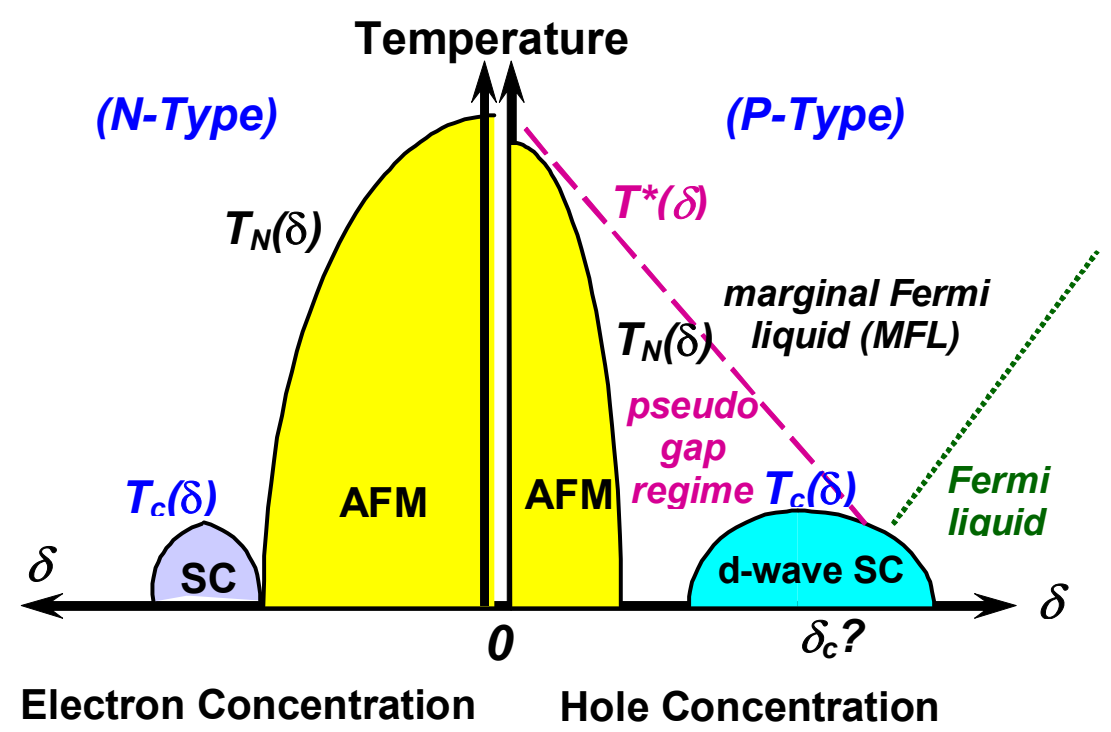

Figure 1.2: Generic temperature $(T)$ vs. doping level $(\delta)$ phase diagrams of $p$-type and $n$-type cuprates in zero magnetic field. (AFM: antiferromagnetic phase; SC: superconducting phase; $T_{N}, T_{c}$, and $T^{*}$ are the Néel, superconducting and pseudogap transition temperatures, respectively.) In the $p$-type superconductor YBCO, the undoped material is an antiferromagnetic insulator with a relatively high Neel temperature (> $200 \mathrm{~K})$. Unpaired electrons at the copper sites in the $\mathrm{CuO}_{2}$ plane undergo superexchange interaction and arrange themselves in alternating directions to form an antiferromagnetic lattice. Upon doping, holes are introduced at oxygen sites, which interact with neighboring copper spins. This interaction competes with the long-range antiferromagnetic ordering. As the kinetic energy of the holes increases with doping concentration, the holes can become delocalized. Thus, as the doping approaches the optimal concentration, the long-range antiferromagnetism is suppressed and the cuprate becames metallic. However, neutron scattering and NMR experiments continue to show strong dynamic antiferromagnetic fluctuations, even in the metallic and superconducting states. This has encouraged theories that suggest this antiferromagnetic background and the spin fluctuations as possible ingredients in mechanisms of pairing [4]. 
superconductivity are substantially different from the phonon-mediated model of conventional systems, and that magnetism may play a very important role in these cuprate superconductors, which are strongly correlated electronic systems, also known as doped antiferromagnetic Mott insulators [5, 6, 7]. One of the most intriguing phenomena associated with the cuprate superconductors is the predominantly $d_{x^{2}-y^{2}}$-wave pairing symmetry in the hole-doped ( $p$-type) cuprates, as manifested by the directional quasiparticle tunneling spectra taken with scanning tunneling microscopy (STM) $[8,9,10,11,12,13]$ and phase sensitive studies using scanning SQUID microscopy [14]. On the other hand, the pairing symmetry in electron-doped ( $n$-type) cuprate superconductors appears to be nonuniversal, ranging from $d_{x^{2}-y^{2}}$ to $s$-wave pairing, depending on the doping level and electronic anisotropy $[15,16,17,18]$. Generally speaking, the physical properties of different families of cuprates are complicated and depend strongly on the doping level, the coupling between $\mathrm{CuO}_{2}$ planes, and disorder. Nonetheless, some generic features have emerged, as exemplified in Fig. 1.2 for the generic phase diagrams of $p$-type and $n$-type cuprates. The complexity associated with the cuprates is in part due to competing orders in the ground state of these strongly correlated electronic materials, which also contribute to the lack of consensus in the theoretical understanding of the cuprates.

\subsection{Motivation}

One of the most intriguing open questions associated with high-temperature superconductivity is the relevance of $d_{x^{2}-y^{2}}$-wave pairing symmetry [8, 9] and antiferromagnetic spin correlation [19] to the pairing mechanism, and the possibility of spin-charge separation 
due to either the resonating-valence-bond (RVB) scenario $[20,21,22]$ or the existence of charged stripes $[23,24]$. A natural consequence of the $d_{x^{2}-y^{2}}$-wave pairing symmetry in the hole-doped ( $p$-type) cuprate superconductors $[8,9]$ is an anisotropic pairing potential and the existence of nodal quasiparticles that are responsible for the unconventional low-energy excitation spectra [5,6], as illustrated in Fig. 1.3. The doping of holes into the oxygen $2 p$-orbitals in the $\mathrm{CuO}_{2}$ planes is known to incur spin fluctuations in the cuprates due to the frustration of nearest-neighbor antiferromagnetic $\mathrm{Cu}^{2+}-\mathrm{Cu}^{2+}$ correlation, and the existence of spin fluctuations has been suggested as relevant to the $d_{x^{2}-y^{2}}$-wave pairing in the cuprates [19]. The antiferromagnetic correlation has also motivated the RVB scenario that could lead to spin-charge separation and the marginal Fermi-liquid (MFL) electronic behavior [25] in the normal state of the underdoped samples. However, to date there has been no direct evidence for spin-charge separation in the cuprates.

The existence of $d_{x^{2}-y^{2}}$-wave pairing and antiferromagnetic correlation is also believed to be responsible for the unusual response of $p$-type cuprates to quantum impurities $[26,27,28,29,30]$. That is, the substitution of $\mathrm{Cu}^{2+}$ by non-magnetic impurities (such as $\mathrm{Li}^{+}, \mathrm{Zn}^{2+}, \mathrm{Mg}^{2+}$ and $\mathrm{Al}^{3+}$ ) in the $\mathrm{CuO}_{2}$ planes of $\mathrm{Bi}_{2} \mathrm{Sr}_{2} \mathrm{CaCu}_{2} \mathrm{O}_{\mathrm{x}}$ and $\mathrm{YBCO}$ systems has revealed stronger pair-breaking effects than the magnetic impurities (such as $\mathrm{Ni}^{2+}$ ) $[31,32,33,34,35,36,37,38,39,40,41,10]$, as illustrated in Fig. 1.4. This is in sharp contrast to the insensitivity of conventional superconductors to non-magnetic impurities $[42,43]$. In light of the unconventional response to static magnetic and non-magnetic impurities and in search of possible evidence for spin-charge separation in the cuprates [44], a feasible experimental approach is to compare the spin and charge transport in the cuprate 


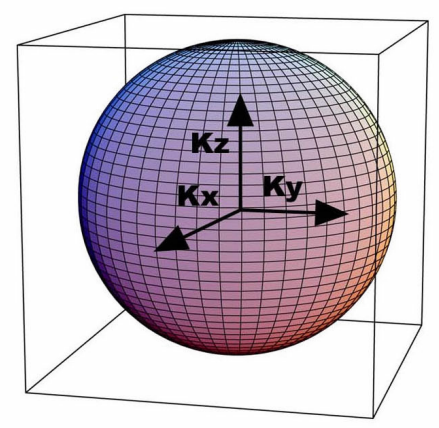

s-wave

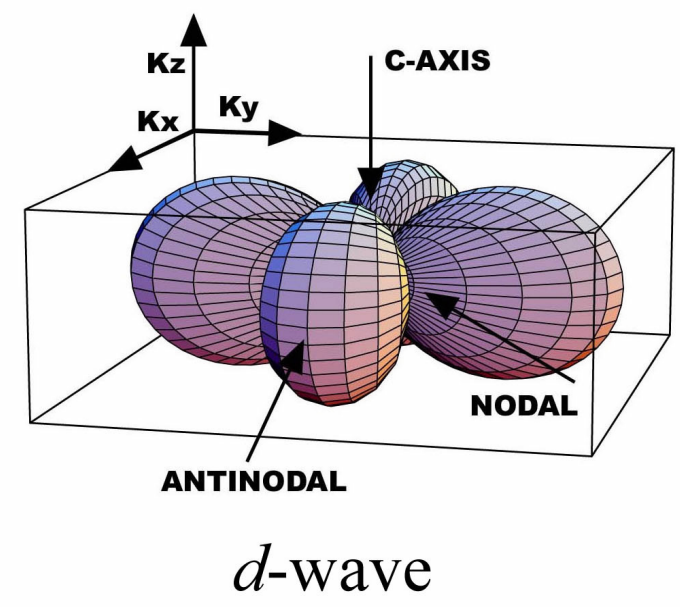

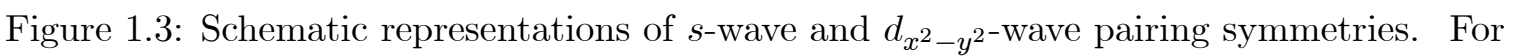
$s$-wave pairing, the superconducting energy gap can be expressed as $\Delta_{k}=\Delta_{0}$, and for $d_{x^{2}-y^{2}}$-wave pairing, $\Delta_{k}=\Delta_{d} \cos 2 \theta_{k}$, where $\theta_{k}$ is the angle relative to the anti-node of the order parameter in $\mathbf{k}$-space [4]. 

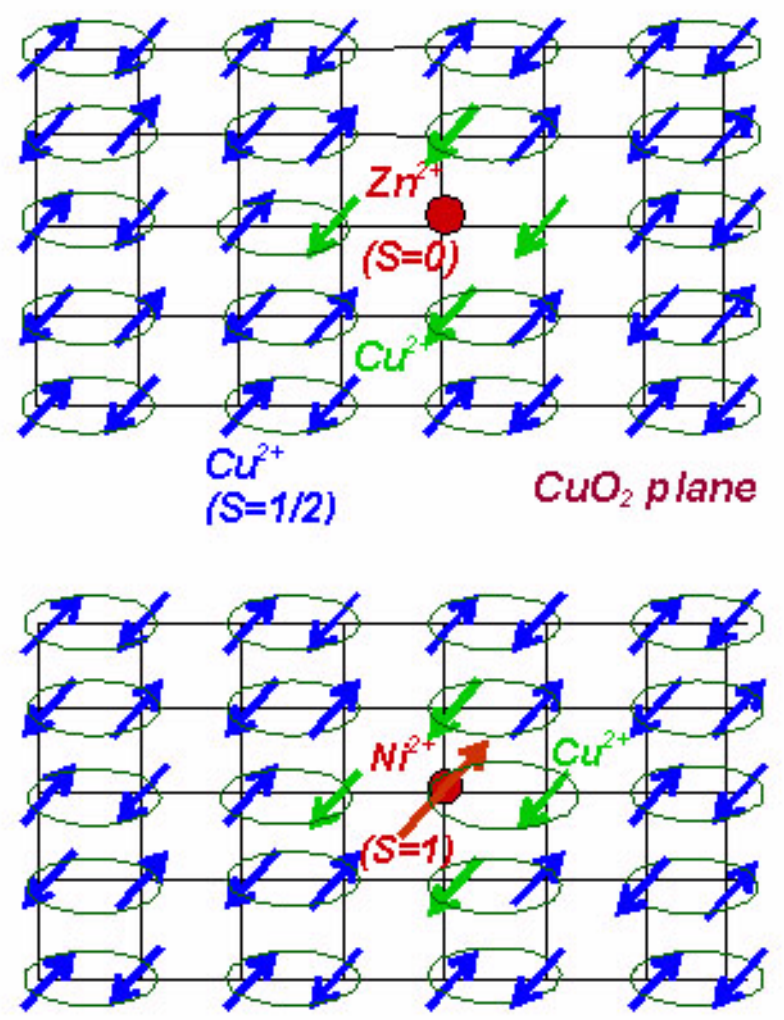

Figure 1.4: Effects of quantum impurities on $p$-type cuprate superconductors in the underdoped limit. In the upper panel, induced magnetic moments on the neighboring $\mathrm{Cu}^{2+}$ sites surround a spinless impurity, such as $\mathrm{Zn}^{2+}$ or $\mathrm{Mg}^{2+}$ with $S=0$. In the lower panel, a localized $\mathrm{Ni}^{2+}$ impurity coexists with the background AFM coupling in the $\mathrm{CuO}_{2}$ plane [4].

superconductors. Such investigation may be conducted by comparing the cuprate response to externally injected simple and spin-polarized quasiparticles, and the physical description for the experimental phenomena would involve concepts of nonequilibrium superconductivity $[45]$.

In the rarely studied case of injection of spin-polarized quasiparticles, two primary effects on the suppression of superconductivity must be considered [46]. One is associated with the resulting excess magnetic moments that break the time-reversal symmetry in singlet 


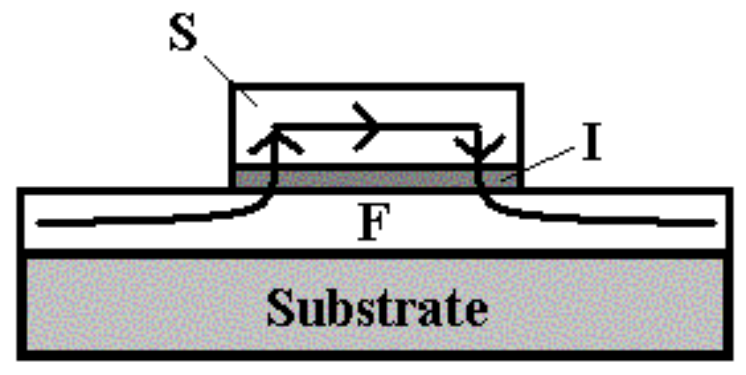

Figure 1.5: Schematic drawing of current injection in a cross-sectional view of an F-I-S heterostructure.

superconductors [47]. The other is the excess momentum and the nonequilibrium quasiparticle distribution that modify the energy spectrum of the superconductor $[48,45]$ if the relaxation of the externally injected quasiparticles is sufficiently slow in the superconductor. In the absence of significant scattering by either magnetic impurities or spin-orbit coupling, the transport lifetime of spin polarized quasiparticles is expected to be much longer than that of simple quasiparticles due to the low probability of pair recombination with other quasiparticles [46]. However, the complexity of the combined effects aforementioned has limited theoretical development at the microscopic level for spin-polarized quasiparticle transport in superconductors.

Spin injection into superconductors can be accomplished by passing electrical currents through a ferromagnet before the tunneling across a thin insulating barrier into a superconductor $[49,50]$, as shown in Fig. 1.5. In recent years, the injection of spin-polarized current in perovskite ferromagnet-insulator-superconductor (F-I-S) heterostructures has attracted significant experimental interest $[51,52,53,54,55]$. This technique utilizes the excellent lattice match among various perovskite materials for epitaxial film growth [53] of the heterostructures, and also takes advantage of the half-metallic ferromagnetism of 
perovskite manganites $[56,57,58,59,60]$ that yield much better spin polarization than typical metallic ferromagnets. Thus, investigating the characteristic spin and charge relaxation and transport processes in the perovskite F-I-S and N-I-S devices can be a unique vehicle for probing nonequilibrium superconductivity and possibly the pairing mechanism in the cuprates. Indeed, strong suppression of the superconducting critical current has been observed in cuprate superconductors by injecting currents from the underlying half-metallic ferromagnetic manganite films $[51,52,61,53,54]$. However, earlier studies of some of the F-I-S devices $[51,52]$ are not conclusive due to complications of Joule heating. Injection of spin-polarized current from ferromagnetic $\mathrm{La}_{2 / 3} \mathrm{Sr}_{1 / 3} \mathrm{MnO}_{3}$ into $\mathrm{DyBa}_{2} \mathrm{Cu}_{3} \mathrm{O}_{7}$ superconductor has been found to strongly suppress the critical current by Vas'ko et al. with efficiency values of unity. Dong et al. later would demonstrate similar suppression in the supercurrent in $\mathrm{YBa}_{2} \mathrm{Cu}_{3} \mathrm{O}_{7}$ with $\mathrm{Nd}_{0.7} \mathrm{Sr}_{0.3} \mathrm{MnO}_{3}$ as the injection source of spin-polarized quasiparticles. The efficiency observed in this particular heterostructure has a reported efficiency of $\sim 3$, which shows promise for future device applications. Both experimental data reveal insignificant effects of simple quasiparticle injection in the control samples, with gold film and perovskite non-magnetic metal serving as the simple quasiparticle injector, respectively. Consequently, the experimental findings are attributed to the dynamic pair-breaking effects of spin-polarized quasiparticles as a result of excess magnetic moments and quasiparticle redistribution $[53,54,55]$.

Despite a significant number of experimental reports that are supportive of the effects of spin-injection in cuprates, many important issues are yet to be resolved. Experimentally, determining the magnitude and temperature dependence of the spin relaxation 
length and time has proven to be elusive. Theoretically, microscopic interactions of externally injected spin-polarized quasiparticles with the Cooper pairs and existing quasiparticles in cuprate superconductors remain unknown. Nonetheless, the intrinsic anisotropy in the cuprate superconducting order parameter due to the predominant $d_{x^{2}-y^{2}}$-wave pairing symmetry $[12,62,10,11]$ and the weakly interacting-layered structure [63] are expected to be relevant to the spin and charge transport. Such anisotropy could give distinctly different mechanisms of spin relaxation in the $a b$-plane and along $c$-axis. For instance, the in-plane simple quasiparticle recombination time $\tau_{R}$ in $\mathrm{DyBa}_{2} \mathrm{Cu}_{3} \mathrm{O}_{7-\delta}$ is found to be significantly longer $\left(\tau_{R} \approx 10^{-6} \sim 10^{-5} \mathrm{~s}\right)$ than the typical values $\left(\tau_{R} \approx 10^{-9} \sim 10^{-7} \mathrm{~s}\right)$ in conventional superconductors. The relaxation time of simple quasiparticles is much longer than that in conventional superconductors because of the tendency of simple quasiparticles to relax towards the zeros of the superconducting gap and also to the reduced scattering rate of nodal quasiparticles by phonons [64]. In addition to the interaction with nodal quasiparticles, quasi-static injection of spin-polarized quasiparticles into the cuprates can suppress the antiferromagnetic correlation in the $\mathrm{CuO}_{2}$ planes, which may result in significant and long-range effects on the cuprate superconductivity, similar to the strong influence of nonmagnetic quantum impurities in the $\mathrm{CuO}_{2}$ planes that induce finite magnetic moments on the nearest-neighbor $\mathrm{Cu}^{2+}$-ions surrounding the impurity site [30].

In this research, nonequilibrium superconductivity is studied by quantifying various characteristics of spin injection in F-I-S heterostructures. By comparing the degree of critical current suppression $\Delta J_{c}$ in the presence of external injection at different $\mathrm{YBCO}$ thickness, a temperature dependence of the $c$-axis spin relaxation length in F-I-S may be 
inferred. Furthermore, an empirically defined efficiency ( $\eta$, which measures the suppression of critical currents due to injected quasiparticles) is studied in detail for both F-I-S and N-I-S systems. The results of this investigation suggest that spin-polarized quasiparticles exert strong effects on suppressing the cuprate superconductivity well beyond the prediction of conventional nonequilibrium superconductivity, probably due to their strong influence on the short-range $\mathrm{Cu}^{2+}-\mathrm{Cu}^{2+}$ antiferromagnetic coupling and the intimate correlation of superconductivity with the background antiferromagnetism.

\subsection{Thesis Overview}

This thesis begins by discussing relevant background of superconductivity in Chapter 2. It includes a brief review of basic physical quantities in superconductors that are relevant to this work and a discussion on the basic concepts of nonequilibrium perturbations in conventional superconductors. After introducing the theoretical background needed for this work, Chapter 3 discusses some of the basic sample preparation techniques as well as laboratory equipment, including cryogenic dewars and probes, used for this work. In Chapter 4 , a digression is made to elaborate on the characterization techniques and measurements done on the CMR material used in the spin injection study. A brief analysis of the obtained data is included in the chapter for completeness. Chapter 5 begins the presentation of the spin-injection experiments and their results on as-grown heterostructures, and more results and comprehensive analysis of the data follows in Chapter 6 . Chapter 7 gives the background information on the thin film processes, which were used extensively in microfabrication of patterned devices. In Chapter 8, a detailed account of the fabrication 
procedures for the patterned injection heterostructures and preliminary results from those devices are presented and discussed. Finally, a concluding summary is included in Chapter 9, and possible future studies are suggested. 


\section{Chapter 2}

\section{Superconductivity Background}

Much of the present day understanding of superconductivity has been provided by the microscopic theory of Bardeen, Cooper, and Schrieffer (BCS, 1957) and the phenomenological theory of Ginzburg-Landau (GL, 1950). A detailed treatment of the theories is beyond the scope of this chapter, thus the readers are referred the readers to Ref. [45]. Instead, discussions on critical currents and topics in nonequilibrium superconductivity will be presented, which are helpful background for experiments in this thesis.

\subsection{Critical Magnetic Field and Critical Current}

Superconductors can be generally divided into type-I and type-II categories. A type-I superconductor exhibits two characteristic properties, namely, zero dc electrical resistance and perfect diamagnetism, when cooled below its critical temperature $T_{c}$ and kept under a thermodynamic critical field $H_{c}$. Perfect diamagnetism, also called the Meissner effect, means that the magnetic susceptibility has the value $\chi=-1$ (in SI units), so that 
an applied magnetic field is excluded from entering a superconductor and a field originally in a normal sample is expelled as it is cooled through $T_{c}$, provided that $H<H_{c}$. The existence of Meissner effect implies that superconductivity will be destroyed if the applied magnetic field exceeds the thermodynamic critical field $H_{c}$. The temperature dependence of the critical field $H_{c}(T)$ can be approximated by

$$
H_{c}(T) \approx H_{c}(0)\left[1-\left(T / T_{c}\right)^{2}\right]
$$

Generally most type-I superconductors are elements such as $\mathrm{Al}$, In and $\mathrm{Pb}$, whereas most type-II superconductors are in the forms of alloys and compounds.

A type-II superconductor is also a perfect conductor, with zero dc resistance, but its magnetic properties are more complex. It totally excludes magnetic flux only in the Meissner state when the applied magnetic field is below the lower critical field $H_{c 1}$. Flux begins to penetrate partially when the applied field is above the lower critical field $H_{c 1}$ and below the upper critical field $H_{c 2}$. The material turns normal for applied fields above the upper critical field $H_{c 2}$. Hence, in the regime of partial flux penetration, the diamagnetism is not perfect. In conventional type-II superconductors, the mixed state is known as the Abrikosov vortex lattice state, with ordered vortices of supercurrents and each vortex containing one flux quantum. In the case of high-temperature superconductors, the large electronic anisotropy and thermal fluctuation effects result in an additional vortex-liquid phase at higher temperatures below $H_{c 2}(T)$, which is separated from the low-temperature vortex-solid phase by a first-order melting line in the clean limit and a second-order glass-like transition in the strong disorder limit.

The existence of a critical magnetic field implies that there is a maximum current 
density that can be sustained by the superconductor, commonly referred to as the critical current $J_{c}$. This is a direct consequence of the existence of the $H_{c}$ because current flow in the superconductor produces a magnetic field. In a long superconducting wire with radius $a \gg \lambda$, a current $I$ produces a circumferential self-field at the surface of the wire of magnitude $H=2 I / c a$ in cgs units with $c$ being the speed of light. One would expect that the superconductivity would be destroyed when this self-field reaches the critical field $H_{c}$. Therefore, the critical current will be $I_{c}=c a H_{c} / 2$, which scales with the perimeter, not the cross-sectional area of the wire. The implication is that the current flows only in a surface layer of constant thickness. From the London and Maxwell equations, the thickness of the surface layer can be shown to be $\lambda$. It follows that

$$
J_{c}=\frac{I_{c}}{2 \pi a \lambda}=\frac{c}{4 \pi} \frac{H_{c}}{\lambda}
$$

This relation can be extended to wires with $a \ll \lambda$, where the current density is nearly uniform and $I_{c}$ is proportional to the cross-sectional area. From the full GL theory, the critical current derived

$$
J_{c}=\frac{c H_{c}(T)}{3 \sqrt{6} \pi \lambda(T)}
$$

differs only by a numerical factor of $\sim 0.5$.

The above derivation relates $J_{c}$ to the intrinsic thermodynamic quantities of the superconductor. However, the empirically determined $J_{c}$ is generally also dependent on extrinsic factors such as flux pinning by defects and anisotropy of crystalline structure. The presence of moving flux affects critical current because the energy that keeps the flux moving is drawn from the supercurrent. Stronger pinning forces would prevent the energydissipating movement of vortices that curbs the supercurrent. Furthermore, crystalline 
anisotropy is an important factor in determining critical current. In cuprate superconductors, the $\mathrm{CuO}_{2}$ layers are separated by charge reservoirs. Thus, it is expected that the critical current is enhanced along these $\mathrm{CuO}_{2}$ planes compared to the weakly-linked $c$-axis direction. Despite the dependence of $J_{c}$ on both intrinsic and extrinsic parameters, in general one can consider $J_{c}$ as a measure of the effective superfluidity and the phase coherence of a superconductor by introducing an effective penetration depth $\lambda_{\text {eff }}(T)$.

\subsection{Nonequilibrium Superconductivity}

Nonequilibrium superconductivity and its associated phenomena have been studied extensively since the 1970's [48], and the primary focus of the research has been on the effects of either simple (i.e., spin-degenerate) quasiparticle injection $[65,66,67,68,69]$ or photon-induced Cooper-pair breaking and quasiparticle redistribution $[70,71,72,73,74$, $75,76]$ in conventional $s$-wave superconductors. The nonequilibrium effects have yielded observation of both enhancement $[70,71,72,73,74]$ and suppression $[65,66,67,68,69,76]$ of superconductivity.

In the studies on conventional superconductors, nonequilibrium effects are known to occur in a superconductor where the electron population is driven out of thermal equilibrium, into either a steady-state dynamic equilibrium or into a time-dependent regime. A dynamic equilibrium is created when a perturbing source is offset by some type of relaxation and diffusion to give rise to a steady state different from thermal equilibrium. Typical examples include the injection of a normal current into a superconductor at an NS interface and the stimulation or weakening of superconductivity by microwave irradiation 
that effectively cool or heat the electrons. In time-dependent nonequilibrium regimes, the magnitude and phase of the order parameter $\psi \sim|\Delta(\mathbf{r})| e^{i \varphi(\mathbf{r})}$ can vary in time as well as in space. Examples include the dynamic behavior of short, superconducting metallic weak links and phase-slip centers in long, superconducting filaments above $I_{c}$ [48].

\subsubsection{Quasiparticle Disequilibrium}

In BCS theory, the energies of single-particle excitations from the superconductive ground state are give by $E_{k}=\left(\Delta^{2}+\xi_{k}^{2}\right)^{1 / 2}$, where $\xi_{k}$ is the one-electron energy relative to the Fermi energy of the state $k$ in the normal state and $\Delta$ is the BCS gap parameter. The probability that these quasiparticle states are occupied is given by the Fermi function

$$
f_{0}\left(E_{k} / k T\right)=\frac{1}{1+e^{E_{k} / k T}}
$$

In the nonequilibrium case, the actual occupation numbers of these states are given by $f_{k}$, which differs from $f_{0}\left(E_{k} / k T\right)$. For the simple spatially uniform case, these $f_{k}$ determine the magnitude of the gap $\Delta$ through the BCS gap equation for an $s$-wave superconductor, which can be written as

$$
\frac{2}{V}=\sum_{k} \frac{1-2 f_{k}}{E_{k}}=\sum_{k} \frac{1-2 f_{k}}{\left(\Delta^{2}+\xi_{k}^{2}\right)^{1 / 2}}
$$

To characterize the effects of perturbation from thermal equilibrium, two parameters $T^{*}$ and $Q^{*}$ are introduced to represent the nonequilibrium temperature and quasiparticle charge density, respectively $[46,77,78,66,67]$. These parameters are derived from the fact that the departure from equilibrium

$$
\delta f_{k} \equiv f_{k}-f_{0}\left(E_{k} / k T\right)
$$


can be decomposed into two orthogonal components that are even and odd with respect to inversion through the local Fermi surface, as illustrated schematically in Fig. 2.1.

The even mode disequilibrium influences both the hole-like and electron-like branches of quasiparticle spectrum equally and has the same effect on the magnitude of the energy gap as a change in temperature. Therefore, the even mode can also be referred to as the "energy", "temperature", or "longitudinal" mode [46]. Neutral perturbations such as phonons or photons can give rise to these even mode excitations in pure form, but charged perturbations such as electron tunneling currents can excite the even mode in conjunction with the odd mode. To quantify the strength of this disequilibrium, an effective quasiparticle temperature $T^{*}$ is defined such that $\Delta_{B C S}\left(T^{*}\right)$ equals the $\Delta\left(f_{k}\right)$. Near $T_{c}$, where $\Delta \ll k T_{c}$, the temperature shift $\delta T^{*}$ is given by

$$
\frac{\delta T^{*}}{T} \equiv \frac{T^{*}-T}{T} \approx \frac{1}{N(0)} \sum_{k} \frac{\delta f_{k}}{E_{k}}
$$

where $N(0)$ denotes the normal-state density of states at the Fermi level.

The odd mode disequilibrium represents a net quasiparticle charge density, represented by

$$
Q^{*} \equiv \sum_{k} \frac{\xi_{k}}{E_{k}} \delta f_{k}
$$

which is generated by charged perturbations, such as electron injection, or conversion of normal current to supercurrent near an interface [66, 67]. Associated with this charge imbalance must be a compensating change in the number of electrons in the condensed BCS ground state. As a result, the electrochemical potentials of the normal quasiparticles $\mu_{n}$ and the superconducting pairs $\mu_{p}$ shift in opposite directions from the equilibrium value $E_{F}^{0}$, in order to maintain overall electrical neutrality [65]. This "branch imbalance" is a 


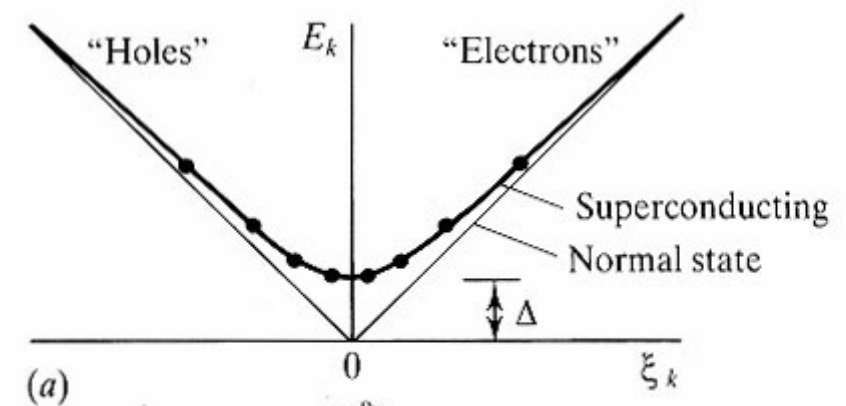

$\left(E_{F}^{0}\right)$

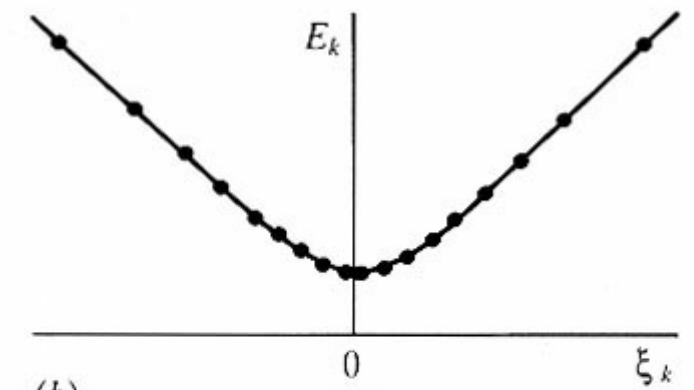

Even mode

(b)

$\left(E_{F}^{0}\right)$

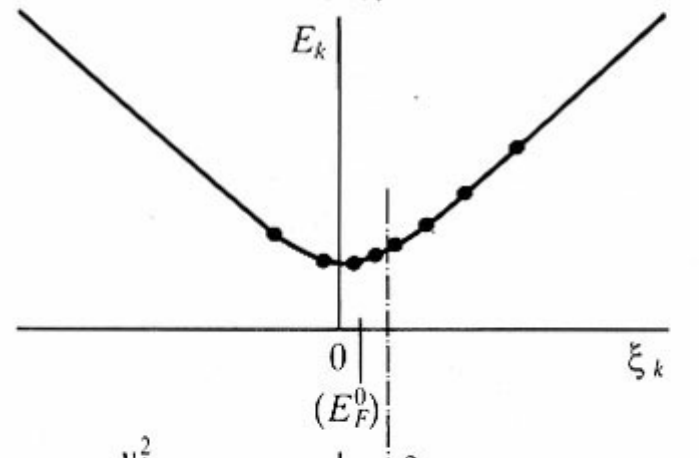

Odd mode

"Branch imbalance"

$Q^{*}>0$

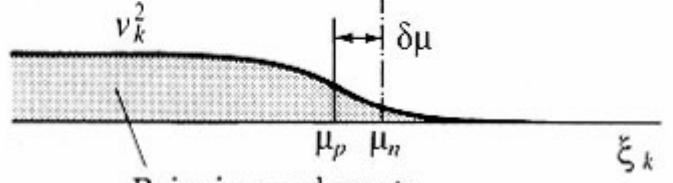

(c) Pairs in condensate

Figure 2.1: a) Dispersion curves of excitation energies in normal and superconducting states. b) Even (or energy) mode excitation, with $T^{*}>T$. c) Odd (or charge) mode excitation, with $Q^{*}>0$ and the shift of $\mu_{n}$ and $\mu_{p}$ relative to $E_{F}^{0}$ [45]. 
measurable effect that is impossible in the normal state because the latter lacks the degree of freedom of the condensate. The odd mode disequilibrium is also referred to as a "charge" or "transverse" mode.

\subsubsection{Relaxation Times}

The relaxation time necessary for the disequilibrium to relax after the given perturbation is terminated often determines the strengths of observable effects by the perturbation. Near $T_{c}$, the relaxation times for the two modes can be written, respectively, as

$$
\begin{gathered}
\tau_{R}^{(L)}=\tau_{\Delta}=\tau_{T^{*}} \approx 3.7 \tau_{E} k T_{c} / \Delta \\
\tau_{R}^{(T)}=\tau_{Q^{*}}=(4 / \pi) \tau_{E} k T_{c} / \Delta,
\end{gathered}
$$

where $\tau_{E}$ is the energy-relaxation (i.e., $\delta f_{k} \rightarrow 0$ ) or inelastic scattering time for an electron at the Fermi surface, usually dominated by electron-phonon scattering [79]. These equations specify that the relaxation times diverge as $\left(1-T / T_{c}\right)^{-1 / 2}$ near $T_{c}$ as the gap parameter $\Delta$ vanishes. The divergence can be understood qualitatively by the argument that, near $T_{c}$, only processes involving the fraction $\sim \Delta / k T_{c}$ of thermally occupied states just above the gap are fully effective in controlling the gap or relaxing the charge imbalance [66, 67, 69].

\subsubsection{Steady-State Energy-Mode}

In the 1970's, Eliashberg first provided the theoretical details in which a steadystate external perturbation introduced in a superconductor lowers the effective temperature $T^{*}$, thus enhancing the superconductivity $[75,76]$. The basic idea is that by suitable choice of values of $\delta f_{k}$, the nonequilibrium shift in effective temperature can be made to be 
negative. This can be achieved in a direct way if the $\delta f_{k}$ can be made to be predominately negative by reducing the total number of quasiparticles preferentially. In addition, even if $\sum\left(\delta f_{k}\right)=0$, the effective temperature shift can be made to be negative as long as the negative values of $\delta f_{k}$ are weighted in states near the gap with lower values of $E_{k}$. The net effects of a negative $\delta T^{*}$ are the strengthening of superconductivity below $T_{c}$ and the enhancement of $T_{c}$ itself.

\section{Enhancement by Microwave Irradiation}

The first experimental demonstration of the energy-mode enhancement was the Wyatt-Dayem effect $[70,71]$, which is the enhancement of the dc critical current of a superconducting bridge by microwave radiation. Klapwijk and Mooij later demonstrated this same effect in long, narrow strips, as well as the enhancement of $T_{c}[72,73,74]$. The enhancement of $\Delta$ was shown by Kommers and Clarke [80].

In these experiments, microwave radiation with photon energy $\hbar \omega<2 \Delta$ was irradiated on a superconducting film to elevate the existing quasiparticles from the low-lying states $\Delta \leq E_{k} \leq(\Delta+\hbar \omega)$ to the less populated states higher in energy by $\hbar \omega$. For higher energy states, downward transition can be stimulated by the microwave irradiation. The photon energy limit is necessary to restrict the generation of new quasiparticles. Therefore, with $\delta f_{k}<0$ for the low-lying states and $\delta f_{k}>0$ for the higher states, cooling occurs in the superconductor according to Eq. 2.7, even though there is a net increase in mean energy of the quasiparticle distribution. Also, an actual decrease in the total number of quasiparticles results because recombination is more rapid at higher energies [81]. It is important to note that there is a minimum frequency for the enhancement effect to take 
place because at lower frequencies, the rf current will only serve to break pairs and weaken superconductivity. The crossover frequency is of the order of $\tau_{E}^{-1}$ [82], below which the photon energies cannot be effectively absorbed before phonon emission such that no changes in $f_{k}$ can take place.

\section{Enhancement by Quasiparticle Extraction}

The energy gap of the superconductor can be enhanced by reducing the quasiparticle density through extraction [83]. Extraction of quasiparticles can be accomplished through a tunneling process between two superconductors with different gap values, biased at voltage $e V=\left|\Delta_{2}-\Delta_{1}\right|$. In such an arrangement, a net flow of quasiparticles from the heavily populated states in the low-gap superconductor to the lightly populated states of the high-gap superconductor will occur. Consequently, $\sum\left(\delta f_{k}\right)<0$ in the low-gap superconductor and its gap value is enhanced. This method of gap enhancement is not limitless; typically, $\Delta_{e q}(T=0)$ of the low-gap superconductor remains the upper bound for the energy gap.

\subsubsection{Steady-State Charge-Mode}

If an electron-like or hole-like excitation is added to a superconductor, a charge imbalance is created. The first quantitative understanding of this charge imbalance arose from an experiment of J. Clarke in which electrons are injected via a tunnel junction into a superconducting film [65]. The steady-state charge imbalance is governed by balancing the local injection rate $Q_{i n j}^{* \prime}$ against an appropriately averaged relaxation rate $Q^{*} / \tau_{Q}$; i.e., $Q^{*}=Q_{i n j}^{* \prime} \tau_{Q^{*}}$. In addition, a measurable electrochemical potential difference $V$ between 
pairs and quasiparticles results from the charge imbalance. It can be shown that the measured potential is given by

$$
V=\frac{Q^{*}}{2 e N(0) g_{N S}}
$$

where $N(0)$ is the usual density of states for electrons of one spin, and $g_{N S}$ is the measured normalized conductance of the normal probe junction [66]. From this expression, an empirical $V$ determines the value of $Q^{*}$.

Furthermore, Clarke also showed that the relaxation time $\tau_{Q^{*}}$ can be determined empirically using the relation $[68,69]$

$$
\tau_{Q^{*}}=\frac{2 N(0) e^{2} \Omega g_{N S} V}{F^{*} I_{i n j}} .
$$

This follows since the charge injection rate $Q_{i n j}^{* \prime}$ differs from the measured injection current per unit volume $\Omega$ only by a known function $F^{*}$, which is usually near unity. From Clarke's data, a value of $\tau_{Q^{*}}$ that diverged near $T_{c}$ is obtained, and $\tau_{E} \sim 10^{-10} \mathrm{~s}$ is extracted for tin. At lower temperatures, the additional relaxation mechanism based on gap anisotropy and elastic scattering must also be taken into account.

In certain configurations, the nonequilibrium population will diffuse away from the injection point while relaxing. If the geometry is such that the diffusion is essentially one-dimensional, $Q^{*}$ will decay as $e^{-x / \Lambda_{Q^{*}}}$, where

$$
\Lambda_{Q^{*}}=\sqrt{D \tau_{Q^{*}}}=\left(\frac{1}{3} v_{F} \ell \tau_{Q^{*}}\right)^{1 / 2}
$$

is the diffusion length for a time $\tau_{Q^{*}}$. Here $D$ is the diffusion constant, $v_{F}$ the Fermi velocity, and $\ell$ the mean-free path. One further expects the associated potential difference between pairs and quasiparticles to decay in a similar way since it is proportional to the 
local value of $Q^{*}$. Therefore, $\tau_{Q^{*}}$ can again be obtained by measuring the diffusion length $\Lambda_{Q^{*}}$, with a known diffusion constant $D$.

\subsection{Summary}

In the injection of spin-polarized quasiparticle experiment in the F-I-S heterostructures, the disequilibrium that results is expected to be charge-mode in nature. Because of the spin polarization of these quasiparticles, the net influx of excess moments into the superconductor is not expected to relax via interactions with phonons. Rather, these quasiparticles must undergo spin-orbit interaction or some type of exchange interaction with other moments. Such spin-flip mechanism must be present to effectively randomize the net spin polarization in order for the quasiparticles to relax and to recombine into the condensate. Therefore, the relaxation time for spin-polarized quasiparticles is expected to be significantly longer than simple quasiparticles. In addition, the single particle energy may incur an effective chemical potential shift, which one can argue to be a theoretical manifestation of the long lifetime of the spin-polarized quasiparticles. 


\section{Chapter 3}

\section{General Experimental Procedures}

Normally, the temperature range of interest in measurements of high temperature superconductivity hovers near the transition temperature of the superconductor, which in the case of $\mathrm{YBa}_{2} \mathrm{Cu}_{3} \mathrm{O}_{7-\delta}$, is typically between 70 and $95 \mathrm{~K}$. However, in spin-injection experiments, the range is expanded to include temperatures from 4.2 to $95 \mathrm{~K}$, where $4.2 \mathrm{~K}$ is limited by the use of liquid helium as the cryogen of choice. In addition, to study the resistive characteristics of the colossal magnetoresistive (CMR) manganites used as the spin injector, one must extend the upper temperature range to room temperature. Such a broad range of temperature requires a cryogenic system that provides adequate heat insulation for the liquid cryogens at low temperatures and sufficient heating power at high temperatures. In the chapter, details of the various cryogenic systems and sample probes employed to accomplish the required experimental conditions will be discussed. 


\subsection{Sample Preparation}

The $c$-axis-oriented tri-layer $\mathrm{F}(\mathrm{N})-\mathrm{I}-\mathrm{S}$ heterostructures used in this work contained $\mathrm{YBa}_{2} \mathrm{Cu}_{3} \mathrm{O}_{7-\delta}(\mathrm{YBCO})$ as the superconductor, $\mathrm{SrTiO}_{3}$ (STO) or yttria-stabilized-zirconia (YSZ) as the insulator, $\mathrm{La}_{0.7} \mathrm{Sr}_{0.3} \mathrm{MnO}_{3}$ (LSMO) or $\mathrm{La}_{0.7} \mathrm{Ca}_{0.3} \mathrm{MnO}_{3}$ (LCMO) as the ferromagnet, and $\mathrm{LaNiO}_{3}$ (LNO) as the non-magnetic metal (S: superconductor; I: insulator; F: ferromagnet; N: non-magnetic metal). A number of devices were studied with different thicknesses of the constituent layers, and for the F-I-S devices, the choice of either LSMO or LCMO did not yield any discernible differences [53]. The thickness of YBCO ranged from $40 \mathrm{~nm}$ to $160 \mathrm{~nm}$, of LSMO or LCMO and of LNO was kept constant at $100 \mathrm{~nm}$, of YSZ was $1.3 \mathrm{~nm}$, and of STO was either $2 \mathrm{~nm}, 3.5 \mathrm{~nm}$, or $10 \mathrm{~nm}$.

The samples are fabricated by R. P. Vasquez at the Jet Propulsion Laboratory using the pulsed laser deposition technique on $(6 \mathrm{~mm} \times 6 \mathrm{~mm}) \mathrm{LaAlO}_{3}(\mathrm{LAO})(001)$ substrates, with either LSMO, LCMO, or LNO as the lower layer and YBCO as the top layer, and the insulator buffering in between. First, the LSMO and LCMO ferromagnet and LNO non-magnetic films are grown by pulsed laser deposition using stoichiometric targets in 100 mTorr of oxygen with the substrate temperature at $700{ }^{\circ} \mathrm{C}$, and are subsequently annealed at $900{ }^{\circ} \mathrm{C}$ in 1 atm oxygen for two hours. The oxygen concentration is believed to be optimized because samples tested with longer annealing times do not yield further increase of the Curie temperature $T_{\text {Curie }}$. Second, these films are then shadow masked using $\mathrm{LaAlO}_{3}$ to reveal a $6 \mathrm{~mm} \times 2 \mathrm{~mm}$ strip (see Fig. 5.1). Third, it is through this exposed strip that a thin layer of STO insulating buffer and the thickness-varying layer of YBCO superconductor are deposited to complete the tri-layer heterostructure. 
The YBCO superconductors are quite sensitive to air and moisture. After extended exposure in air, they form a non-stoichiometric surface layer that can be removed by a $\mathrm{Br}_{2}$ chemical etch process [84]. Thus, in order to avoid subjecting the thin YBCO films to such etch process, the samples are stored in a vacuum desiccator which contains a fresh desiccant, commonly known as Drierite. Such sample storage condition has been able to successfully maintain the quality of these samples, with no appreciable degradation to the transition temperature $T_{c}$ of the YBCO layer even after one and a half years.

To make electrical contacts, the samples must again be shadow masked in preparation for evaporating or sputtering of gold. Sputtering gold directly on the top surface of the sample is preferred over evaporation because sputtering deposition entrenches the gold atoms further into the sample which results in better adhesion of the contact layer. In contrast, in order for evaporation of gold contacts to be effective, a thin $2 \sim 5 \mathrm{~nm}$ layer of either chromium or titanium should be pre-evaporated on the sample prior to the gold deposition to facilitate adhesion. The shadow mask is cut out of aluminum foil exposing only the gold contact regions. On the YBCO bar, four square contacts are created in a linear formation. It is important to point out that the outer squares should be inside the length of the YBCO strip to avoid undesirable shorting of the gold contact directly with the underlying CMR material. Then, two strips are cut out on each side of the YBCO bar for a total of four contacts on the CMR material. The long strip geometry of the gold contact on the CMR is to ensure the uniformity of current flow in this resistive layer.

Before mounting the sample into the probe, the sample is first set and stabilized with silver paint on a 1/2-inch round sapphire substrate, which is chosen because of its high 
thermal conductivity to minimize thermal gradient between the sample and the stage. Only a sparing amount of the wet silver is applied on the underside of the sample for adhesion to avoid accidental shorting between layers in the sample thus the creation of additional paths for the multiple currents employed in the measurements.

To attach leads to the sputtered gold contacts on the sample, thin indium wire is pressed on both the gold pads and the sapphire substrate. Indium is very malleable at room temperature and can easily be spread using a sharp plastic tool. It is also slightly tacky, which allows it to affix to clean metallic surfaces. The key to a stable bond with low contact resistance is the cleanliness of the gold surface, indium, and most importantly, the tools used to make the bond. The sapphire substrate, the indium wires, and the tools, such as scalpels, tweezers, glass slides, and a sharp plastic instrument for spreading the indium, need to be thoroughly cleaned with organic solvents, in the sequential order of acetone, 2-propanol, and ethanol. Although pressing the indium wires on the tiny contact pads requires steady hands, the bonds have proven to be highly reliable and generally exhibit lower contact resistances $(<1 \Omega)$. Finally, stripped 0.002-inch copper wires are attached to the indium pads on the sapphire substrate. These copper wires are the the exiting connections to the electrical pins when the sample is mounted on the stage of the probe.

\subsection{Cryogenic Dewars}

The low-temperature experiments require a well-defined sample environment. Temperature should stabilize to better than $\pm 0.005 \mathrm{~K}$, and a high dc magnetic field up to $H \sim 9$

Tesla should be available for experiments with CMR materials. For the high magnetic 
fields, a cryogenic dewar with a built-in superconducting magnet is used. In the event that a lower magnetic field, $H \sim 0.1$ Tesla, is needed, a copper coil, water-cooled electromagnet, with maximum field strength of 0.6 Tesla, is employed in another cryogenic dewar. The superconducting magnet, in this case, does not provide accurate small dc magnetic fields on the order of 0.1 Tesla, which could be below the residual field from trapped fluxes of some superconducting magnets. The side benefits from the use of a coil electromagnet include the availability of low magnetic fields with better field resolution through a wider range of temperature below room temperature and the cost savings from not having to cool the superconducting magnet with liquid helium.

\subsubsection{Superconducting Magnet Dewar}

The superconducting magnet system, as shown in Fig. 3.1, contains a single solenoid made of $\mathrm{Nb}_{3} \mathrm{Se}$ superconducting wire placed in the liquid He reservoir. The sample is inserted into the center 2-inch bore and positioned in the middle of the solenoid for maximum uniformity of the magnetic flux lines. Because the superconducting magnet must remain in the liquid He bath during persistent current mode, the sample region is cooled through a weak thermal link to the LHe bath to retain temperature control. To achieve the weak thermal link, the sample space is enclosed by a stainless steel tube, which extends all the way to the top of the dewar, and a vacuum jacket, which extends approximately 15 inches above the sample. Roughly 0.1 mbar of He exchange gas, measured when the dewar is at room temperature, is introduced into the sample space to provide limited heat exchange for temperature control.

There are several notable ways to increase the efficiency of LHe consumption for 

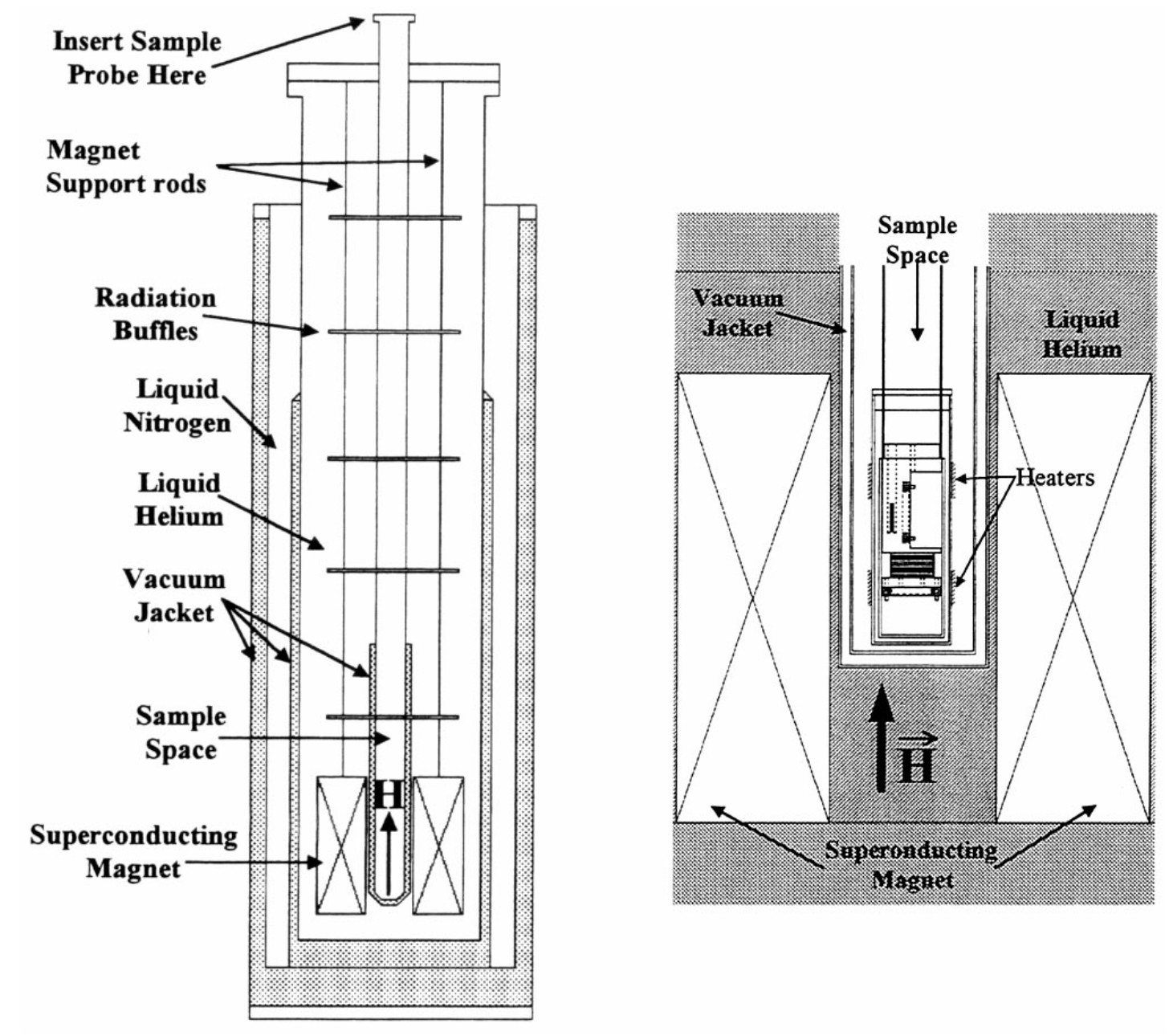

Figure 3.1: Superconducting magnet dewar and the enlarged view of the sample space, vacuum jacket, and magnet solenoid [85].

this large low-temperature cryostat. An efficient procedure to cool down the dewar and the superconducting magnet is by pre-cooling the entire system with liquid nitrogen in the outside reservoir to $T \sim 77 \mathrm{~K}$ and leave the center reservoir over-pressurized with He gas. Also, the addition of several levels of radiation baffles inside the liquid He reservoir reduces the radiative and convective heat from the top of the dewar, which is at room temperature. The metallic leads that supply the current to the superconducting solenoid 
are highly thermally conducting, so they have been designed to detach from the magnet when magnetic field control is not required.

\subsubsection{Low-Field Magnet Dewar}

In the low-field electromagnet dewar system, as shown in the left panel of Fig. 3.2, the sample is place near the base of a narrow extension, which is inserted into the gap space of a self-contained, standard copper coil magnet at room temperature. This magnet provides a horizontal dc magnetic field up to $6 \mathrm{kOe}$. Because of this unusual narrow extension of this dewar, designed to fit the electromagnet, a "super varitemp" temperature control system built by Janis Research Co. is used to provide the necessary cooling mechanism for temperature control. The key to this design is the active cooling resulting from vaporized He entering the sample region through a small capillary tube connected directly to the LHe reservoir (see Fig.3.2, right panel). The flow of liquid helium is controlled by adjusting a needle valve from a knob outside of the dewar. The needle valve cannot control the flow of LHe consistently over an extended period of time as it is dependent on the level of LHe in the reservoir and the vapor pressure in the sample space. Thus, adjustments to the needle valve may be necessary to maintain steady cooling. Since the He vapor cooling the sample is at a temperature near $4.2 \mathrm{~K}$, to avoid large temperature gradient near the sample region, it is determined that the use of LHe is not necessary for measurement temperatures above 85 K. A liquid nitrogen reservoir surrounds the center He reservoir, and can cool the He gas to a temperature near $77 \mathrm{~K}$. By slightly over-pressuring the helium reservoir with helium gas instead of liquid helium, the cold He gas can flow through the capillary to provide sufficient cooling for sample temperature control above $85 \mathrm{~K}$. It is important to monitor 


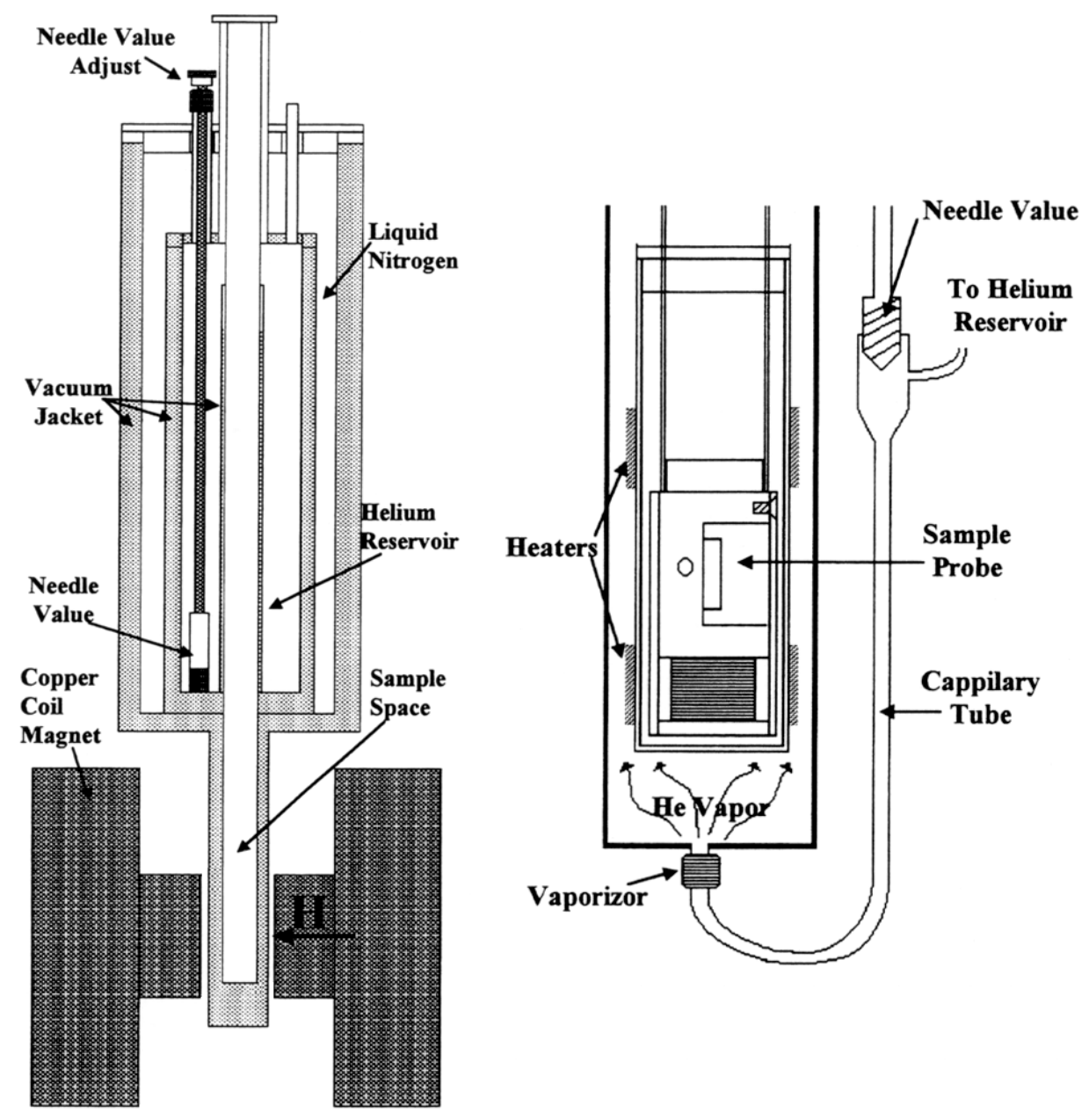

Figure 3.2: Low-field magnet dewar and the enlarged view of sample space and the "super varitemp" temperature control system [85].

the state of the vacuum jacket surrounding the sample space, as the narrow extension does not allow for the usual cold reservoir buffer with room temperature air. With any sign of moisture condensation on the outside of the dewar, the vacuum in the jacket should again be evacuated and restored via a turbo-pump for at least an hour. 


\subsubsection{Liquid Helium Dewar}

For experiments where the presence of a magnet is not needed, a wide-mouth 60 L dewar, with a 2-inch wide neck, supplied by the LHe vendor, Air Products, Inc. can substitute as a cryogenic dewar using a specially designed probe. This method provides several distinct advantages to the previously described cryogenic dewar systems. First, the probe design for "dipping" directly into the LHe dewars is vacuum sealed and has its own built-in vacuum jacket, therefore, the time required for each thermal cycle, i.e., each time the system undergoes cooling and warming, is significantly shortened because only the probe itself, not the entire cryogenic dewar, is cooled and warmed to complete the cycle. Second, effective temperature control throughout a wide range of temperature is easily achieved within a short time without needing to change the condition of the probe or the dewar. This is made possible because of the ability to manually adjust the position of the sample area relative to the LHe surface. For lower temperature measurements, one can submerge the sample space entirely in LHe, and for higher temperature measurements, particularly those above $100 \mathrm{~K}$, the probe can be lifted above the LHe level, with the flexibility of small adjustments to the position of the probe, to avoid high power output from the heaters in the probe for high-temperature control. Third, the amount of helium usage per thermal cycle is minimized because the thermal mass of the sample probe being cooled is small.

\subsection{The Sample Probes}

A sample probe is designed to serve two major purposes: to house the sample in the intended environment of the measurement, and to establish connections with the 
external instrumentation for data collection. Therefore, it must provide rigid physical support, efficient temperature control, and low-noise electrical connection. The probe must also be vacuum sealed to allow for the evacuation of air and any moisture when it is inserted and sealed in the sample space of the dewars. A good vacuum seal will prevent air from leaking in causing moisture condensation inside the probe during cool down, which could result in unreliable temperature control at best, and degradation of the sample as well as cracks or other damage in the sample insert of the cryostat at worst. A slow leak in the probe when using the low-field dewar is tolerable because the varitemp control system maintains a He pressure slightly higher than atmospheric inside the sample space, thus there would be a net outflow of He gas.

Fig. 3.3 shows the general design for the top section of the probe that is positioned outside of the dewar. Vacuum-tight hermetically sealed BNC feedthroughs are placed on the side panels. These feedthroughs, which retain a complete coaxial path through the vacuum seal of the probe, are connected to miniature coaxial wires on the inside. Additional electrical connections for the temperature sensor and heater are provided by a single 19-pin connector. The signal for the 4-lead temperature sensor is transmitted by a miniature quadtwist wire to minimize thermal conduction from the ambient to the sample area, while the current driving the heater is carried by a pair of high gauge copper wire to prevent significant Joule heating when heater currents is turned on. All the feedthroughs are sealed to the top of the probe by Viton O-ring or by non-reusable indium wire. A stainless steel tube extends as the shaft of the probe to the sample stage. 


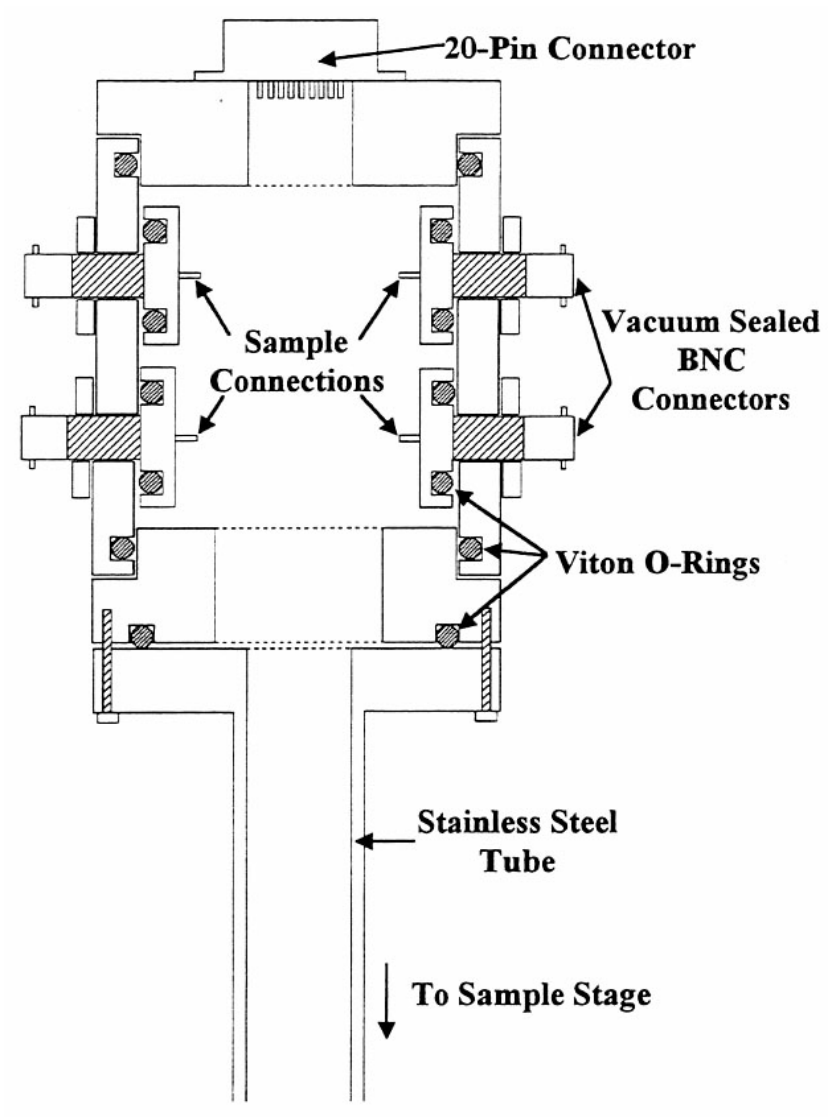

Figure 3.3: Top section of sample probes with vacuum sealed coaxial connectors and feedthrough for temperature sensor and heater [85].

\subsubsection{Standard Transport Probe}

The sample stage is machined from a copper block and has two sample positions for consistent magnetic field orientation in the superconducting magnet and low-field dewars (see Fig. 3.4), which have vertical and horizontal $H$ field directions, respectively. When the sample is placed in the superconducting magnet dewar, the center position would normally provide a field direction parallel to the $a b$-plane of the sample, while the side position would have $H$ along the $c$-axis. However, when placed in the low-field dewar, the center sample position is able to attain an $H$ field parallel to either the $c$-axis or an $a b$-plane direction 


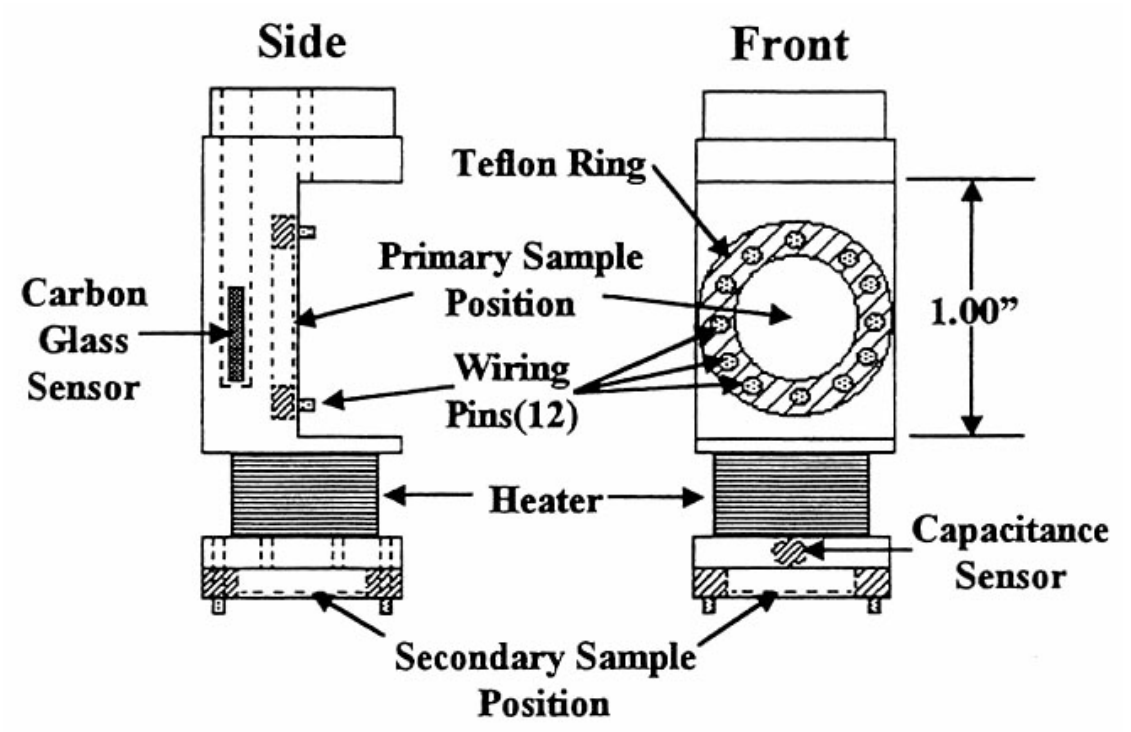

Figure 3.4: Sample stage for standard transport probe [85].

through a $90^{\circ}$ rotation about the axis of the transport probe.

In the sample stage, a carbon-glass temperature sensor is placed directly below the center sample position, and a capacitance sensor is placed directly next to the side position. The sample stage is covered with a copper shield to provide a more uniform temperature for the sample environment. A second shield may be used to further screen the cold He vapor from entering the sample area during the active cooling in the lowfield dewar. An optional heater foil is attached to the outside of this outer shield; when connected to an external current source, coarse temperature control can be maintained. For fine temperature control, a small heating foil on the sample stage is used and controlled by a LakeShore $91 \mathrm{C}$ or $93 \mathrm{C}(\mathrm{A})$ temperature controller. This heater position, located to the side of the center sample position and nominally equidistant from both the sample and the carbon-glass sensor, minimizes the temperature gradient between the sample and sensor, 
and as a result reduces the error in the temperature readout. In high field measurements, temperature control with the capacitance sensor is preferred because its performance shows little dependence with change in magnetic field, although the absolute temperature reading of the capacitance sensor must be calibrated against that of the carbon-glass sensor in zero field. With this probe, temperature stability of $\pm 3 \mathrm{mK}$ can routinely be achieved, with the stability limited by the resolution of the temperature controller.

Copper pins surround the center position on the sample stage, where the sample on sapphire substrate will be placed. These pins are screwed into a ring of teflon to provide electrical isolation to the sample stage. Electrical contacts are made to the sapphire substrate via fine 0.002-inch copper wires, which are soldered to these pins and secured onto the substrate with indium. Miniature coaxial cables are soldered to the copper pins and extend to the top of the probe, connecting directly with the BNC feedthroughs on the side panels. By running the coaxial cables to the copper pins from the top of the probe, the coaxial shielding is maintained as close to the sample as possible to minimize electrical noise, leaving only a short length $(\sim 1 \mathrm{~cm})$ of unshielded wire. The connections of the inner and outer conductors of the coaxial cables to copper pins also serve as thermal anchors that reduce excess thermal load directly to the sample.

\subsection{2 "Quick-Dip" Probe}

The "Quick-Dip" probe, designed and built for this thesis work, is very similar in its internal structure to that of the transport probe. To design a probe to fit directly in the wide-mouth LHe dewar, it needs to withstand the stresses associated with undergoing thermal cycles, therefore, a self-contained vacuum jacket should be incorporated to the 
probe. To accomplish this objective, two thick-walled stainless steel tubes of different diameters are placed concentrically, with the gap between them serving as the vacuum space. At the sample stage, two brass canisters are used to cover the sample space and the vacuum jacket. Because this part of the probe will be submersed into liquid helium, these canisters are sealed with soft indium wires to preserve the vacuum as the probe is cooled. To make it easier to remove and recycle these indium wire O-rings after each use, a coating of Apiezon low-temperature vacuum grease is applied to the indium before each seal.

The strict 2-inch clearance at the neck of the dewar and the addition of the vacuum jacket require the tightening of the dimensions for the sample stage. The bulk stage is again machined out of a copper block, but with only one flat sample position carved out in the center. Four copper posts just outside of the pins are added as thermal anchors for the miniature coaxial cables before they are connected to the pins and the contacts on the sample. A Cernox temperature sensor, with similar performance to carbon-glass sensors, is used for the temperature reading and is located to the side of the sample stage. Two $20 \Omega$ heater strips connected in series are attached below the sample position using diluted GE varnish to provide heating for temperature control. 


\section{Chapter 4}

\section{CMR Material and DC Transport}

Before discussing the spin-injection experiments, it is useful to devote some effort to understand the properties of the CMR material used as the source of spin injection in the heterostructures. Both $\mathrm{La}_{0.7} \mathrm{Sr}_{0.3} \mathrm{MnO}_{3}$ and $\mathrm{La}_{0.7} \mathrm{Ca}_{0.3} \mathrm{MnO}_{3}$ are known to be halfmetallic ferromagnetic materials $[56,57]$ with strong electronic correlations and significant electron-phonon coupling, characterized by the presence of an energy gap for one of the spin orientations at the Fermi level and continuous bands for the other. The basic structure of these manganites belongs to the $\mathrm{ABX}_{3}$ perovskite structure, as shown in Fig. 4.1. An important consequence of the half-metallicity in these materials is the nearly complete spin polarization for the itinerant bands in the ferromagnetic state, which is in contrast to the typical Stoner ferromagnets and is one of the main reasons for choosing them to be the spininjection sources. Given the half-metallic ferromagnetism, it is not difficult to understand the occurrence of CMR effect. As shown in Fig. 4.2, the randomized domains that exist below the Curie temperature $T_{\text {Curie }}$ at $H=0$ will align themselves in the direction of 


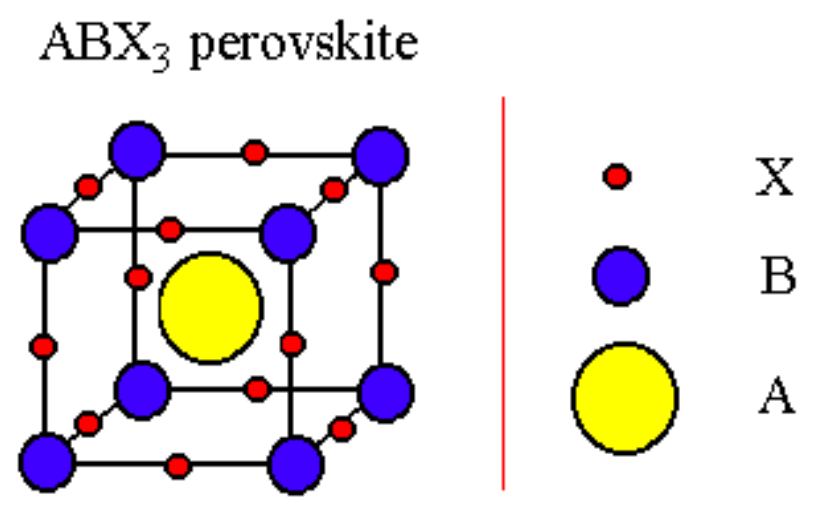

Figure 4.1: Unit cell structure of perovskite $\mathrm{ABX}_{3}$.

the external magnetic field $H$ when it is introduced, thus reducing the spin-dependent energy barrier for transporting conduction electrons across the domain boundaries. It is also necessary to understand certain effects, such as the substrate-induced lattice distortion that yields strong effects on the electrical and magnetic properties of the CMR materials due to the strong electron-phonon coupling, and their influence on properties of the CMR material. Such understanding will be helpful for preparing high-quality samples for the spin-injection experiment. In this chapter, the focus will be placed on investigating these substrate-induced effects in CMR films.

\subsection{Background}

In recent years, colossal negative magnetoresistance (CMR) have been discovered and studied in the perovskite manganites $\mathrm{Ln}_{1-\mathrm{x}} \mathrm{A}_{\mathrm{x}} \mathrm{MnO}_{3-\delta}$ (Ln: trivalent rare-earth ions; A: divalent alkaline-earth ions) systems $[86,87,88,89,90,57]$. It is known that the magnetic phases and electronic properties of these manganites vary with the doping level 


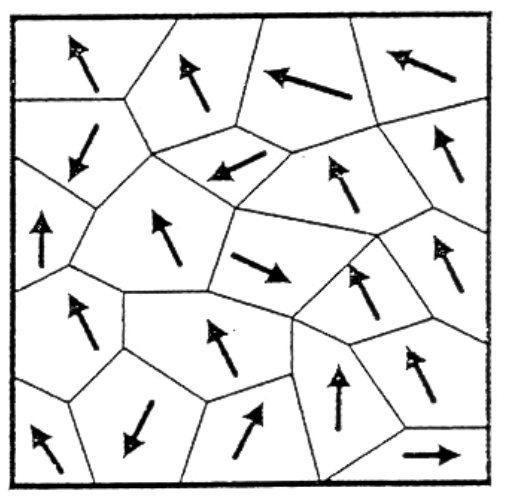

$\mathrm{T}<\mathrm{T}_{\text {Curie }}$ $\mathrm{H}=\mathbf{0}$

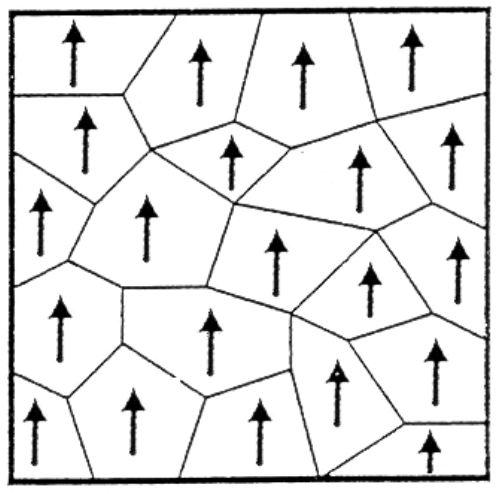

$\mathrm{T}<\mathrm{T}_{\text {Curie }}$ $\mathrm{H}>\mathbf{0}$

Figure 4.2: The alignment of domains in the direction of the external magnetic field $H$.

(x) $[91,92,93,94,95]$. With increasing $x$, the concentration of $\mathrm{Mn}^{4+}$ increases, giving rise to a mixture of $\mathrm{Mn}^{3+}$ and $\mathrm{Mn}^{4+}$, which initially yields canted spin configurations and then forms metallic bonding and ferromagnetism for doping levels in the range $0.2 \leq x \leq 0.4$ $[91,92,93,94,95]$. The occurrence of ferromagnetism had been attributed to the doubleexchange interaction between $\mathrm{Mn}^{3+}$ and $\mathrm{Mn}^{4+}$ ions $[91,92,93,94,95]$. However, further theoretical investigations revealed that the double exchange alone cannot quantitatively account for the observed CMR effect, and that the strong electron-phonon interaction arising from the Jahn-Teller splitting may be important $[88,96]$. The suggested relevance of the lattice effects on the conductivity and magnetism of these manganites is supported by increasing experimental evidence: a strong correlation between the thickness of epitaxial films and the corresponding magnetoresistance has been shown in $\mathrm{La}_{0.7} \mathrm{Sr}_{0.3} \mathrm{MnO}_{3}$ $[89,97]$, where the magnitude of negative magnetoresistance is substantially reduced for film thickness larger than a critical thickness within which the lattice distortion is relaxed; 
decreasing Curie temperatures and increasing $\mathrm{CMR}$ in $\mathrm{La}_{0.7-\mathrm{x}} \operatorname{Ln}_{\mathrm{x}}^{\prime} \mathrm{Ca}_{0.3} \mathrm{MnO}_{3}\left(\operatorname{Ln}^{\prime}=\operatorname{Pr}\right.$, $\mathrm{Y} ; 0 \leq x \leq 0.7)$ polycrystalline materials with decreasing lanthanide average ionic size have been demonstrated via the substitution of smaller ions of $\mathrm{Pr}$ and $\mathrm{Y}$ for the La [57]; studies of $\mathrm{La}_{0.6} \mathrm{~Pb}_{0.4} \mathrm{MnO}_{3}$ and $\mathrm{Nd}_{0.6}\left(\mathrm{Sr}_{0.7} \mathrm{~Pb}_{0.3}\right)_{0.4} \mathrm{MnO}_{3}$ single crystals have illustrated magnetoresistance much smaller than that in the polycrystalline samples [98]; a significant reduction of the magnetoresistance has been observed in $\mathrm{Nd}_{0.5} \mathrm{Sr}_{0.36} \mathrm{~Pb}_{0.14} \mathrm{MnO}_{3}$ single crystals under a hydrostatic pressure of $10.7 \mathrm{kbar}$ [90]; and a large magnetovolume effect [99] and a giant oxygen isotope effect [100] have been found in polycrystalline samples of $\mathrm{La}_{1-\mathrm{x}} \mathrm{Ca}_{\mathrm{x}} \mathrm{MnO}_{3}$.

\subsection{Material and Substrate Selection}

To better understand the roles that lattice distortion and Jahn-Teller effect play in the occurrence of $\mathrm{CMR}$ in the manganites, the transport and magnetic properties of $\mathrm{La}_{0.7} \mathrm{Ca}_{0.3} \mathrm{MnO}_{3}$ and $\mathrm{La}_{0.5} \mathrm{Ca}_{0.5} \mathrm{CoO}_{3}$ epitaxial films on various perovskite substrates are studied. The substrates selected are single-crystalline (001) $\mathrm{LaAlO}_{3}(\mathrm{LAO}), \mathrm{SrTiO}_{3}$ (STO), and $\mathrm{YAlO}_{3}(\mathrm{YAO})$. These substrates are chosen to provide a range of lattice constants, as shown in Table 4.1, which allows studies of the effects of tensile and compressive stress of the films. By maintaining the same chemical composition, oxygen annealing condition, and film thickness for all samples, the net effect of substrate-induced lattice distortion on the transport and magnetic properties can be probed. To investigate the relevance of the Jahn-Teller coupling, the cobaltite $\mathrm{La}_{1-y} \mathrm{Ca}_{\mathrm{y}} \mathrm{CoO}_{3}$, which is known to be highly conductive ferromagnets at doping levels of $0.4 \leq y \leq 0.6[94,101]$, is selected. The $\mathrm{Co}^{3+}$ and $\mathrm{Co}^{4+}$ ions in these cobaltites are known to exist in the form of both high-spin and low-spin states 
with either entirely empty or half-filled $e_{g}$ orbitals [94], which are responsible for the high electrical conductivity and ferromagnetism [102, 103]. However, neither the empty nor the half-filled $e_{g}$ orbitals give rise to Jahn-Teller effect. This absence of Jahn-Teller effect coupled with the high mobility of the conduction electrons renders the electron-phonon interaction in the cobaltites far less significant than that in the manganites.

\subsection{Sample Growth and Characterization}

The $\mathrm{La}_{0.7} \mathrm{Ca}_{0.3} \mathrm{MnO}_{3}$ (LCMO) and $\mathrm{La}_{0.5} \mathrm{Ca}_{0.5} \mathrm{CoO}_{3}$ (LCCO) epitaxial films are grown at the Jet Propulsion Laboratory by pulsed laser deposition using the corresponding stoichiometric targets. The films are deposited in 100 mTorr of oxygen with the substrate temperature at $700{ }^{\circ} \mathrm{C}[58]$. The oxygen concentration is believed to be stoichiometric because additional annealing times do not yield further increase of the Curie temperature $T_{\text {Curie }}$, and the $T_{\text {Curie-values for all LCMO }}\left(T_{\text {Curie }}=260 \pm 10 \mathrm{~K}\right)$ and LCCO $\left(T_{\text {Curie }}=\right.$ $180 \pm 5 \mathrm{~K})$ films on different substrates are consistent with those for the bulk material, as determined from low-frequency magnetic susceptibility measurements made using standard lock-in technique. The thickness of all samples is $200 \pm 10 \mathrm{~nm}$, and the lattice constants $a, b$, and $c(c \perp$ sample surface) as well as the epitaxy of the films are determined using high-resolution x-ray diffraction and x-ray rocking curves. The chemical properties of these samples are characterized with x-ray photoelectron spectroscopy (XPS) [104], and the results indicate that no interdiffusion takes place between the substrate material and the film except in the LCCO/STO film. Moreover, tunneling spectroscopic studies of these manganites using a variable temperature scanning tunneling microscope (STM) reveal no 
density of states at the Fermi level for the manganites at room temperature and zero field, in agreement with the semiconducting nature of these samples above $T_{C u r i e}$. In contrast, a high density of states at the Fermi level is observed for the cobaltites at room temperature [104], which is consistent with their metallic nature.

\subsection{CMR Sample Preparation}

The sample preparation for the CMR films involves making contacts and sample mounting similar to that of the spin-injection samples discussed in the previous chapter. The basic technique in resistivity measurements is to employ a four-contact measurement. For thin films of homogeneous thickness (i.e., without any isolated holes), it is customary to apply the van der Pauw correction in the measurement. This correction is useful especially for samples of arbitrary shape, as long as the four contact probes are sufficiently small and located at the periphery of the sample. The CMR films are grown on square substrates $(6 \times 6 \mathrm{~mm})$, so initially the four contacts where conveniently placed at the four corners of the square film, where the usual multiple pairs of measurements necessary in the van der Pauw method can be reduced down to two. However, through empirical testing, it is determined that this configuration of contacts is inappropriate for the resistivity characterization measurement for these CMR films, especially for the semiconducting LCMO films, which can have resistance signals peaking near several hundreds of $k \Omega$. The van der Pauw correction breaks down in this case because it assumes the sample to be conducting and the flow of measurement current to be homogeneous. In the CMR films, even for films with homogeneous thickness, the current that flows through a highly resistive material with 
spatial variations in the conductance, whether extrinsic or intrinsic, can be significantly non-uniform. The non-uniform current flow sometimes results in artificial readings of negative resistance in some samples if the electrical contacts are placed at the corners of the sample, and the signals for each measurement becomes highly dependent upon the locations of the contact placements.

It is determined that the most suitable configuration for the resistivity measurement of these CMR film is having four elongated parallel strips of contacts deposited across the entire length of the film. In this geometry, a net current with a well-defined current direction is guaranteed, and any effects from the current non-uniformity would already be averaged away.

The samples are mounted onto the probe by attaching it to a sapphire substrate. Again, the contacts are made by pressing indium wires directly onto the gold contacts on the surface of the samples and thin copper wires, which are soldered to the pins located on the stage.

\subsection{Transport Resistivity Measurement}

The 4-contact point measurement is the preferred in resistivity measurements because it accurately accounts for the true resistance signal from the sample by removing the contribution from the serial contact resistances that exist between the sample and the measuring instrument. Here a current supply may be connected to the outer contacts, and a voltmeter to the inter contacts. As the current flows through the circuit, given a sufficiently

large input impedance in the voltmeter, it will stay primarily in the current path defined 
by the sample. Without discernible currents passing through the inner contact resistances, the voltmeter will only detect the voltage drop across the sample between the two inner connectors, thus providing a more accurate resistance measurement on the sample.

The transport resistivity measurement setup includes a nano-voltmeter and a current source. However, rather than connecting the inputs/outputs of the instruments directly to the $\mathrm{BNC}$ connectors at the top of the measurement probe, the wirings are bridged by a 10-channel scanner box. This scanner serves as a switchbox, which allows the user to perform different measurements on permutations of the 4 contacts (useful when longitudinal and Hall resistivity are measured) or on multiple samples. The internal wiring can be custom adjusted by the user for the intended purposes in a given experiment.

\subsection{Magnetization and Susceptibility Measurement}

To link the magnetic properties of the CMR materials to the transport properties, it is important to have access to magnetization and susceptibility measurement techniques. For CMR magnetization, a commercially available SQUID magnetometer is used. The SQUID system is kept in liquid helium during operation, so one has a full range of temperature available for the measurement, from room temperature down to $\sim 1 \mathrm{~K}$. The temperature control of the system operates internally and is controlled by an interface computer for its users. Although it contains a feedback loop when setting temperatures, the system typically requires several minutes of equilibration time, especially when the incre-

ment setting is large. Included in the system also is a build-in high-field superconducting magnet, capable of fields up to $\sim 6$ Tesla. The computer interface provides the command 

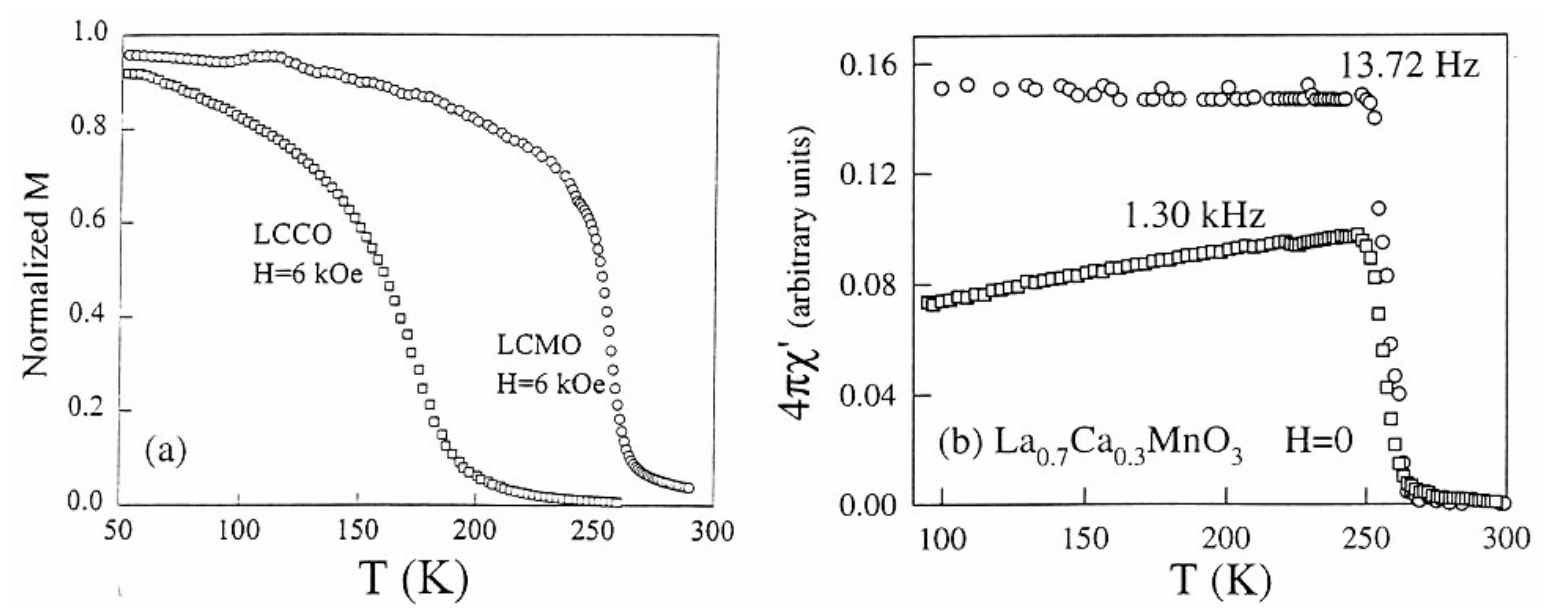

Figure 4.3: a) The magnetization $(M)$ vs. temperature $(T)$ data for bulk LCMO and LCCO samples. b) The real part of the ac magnetic susceptibility $\left(4 \pi \chi^{\prime}\right)$ vs. temperature $(T)$ data taken at frequencies $f=13.72 \mathrm{~Hz}$ and $f=1.30 \mathrm{kHz}$ and for $H=0$ [105].

control and programming ability for measurement scans using the SQUID. To set up for the measurement, the sample is place in a straw holder, which has the length to physically move relative to the two pick-up coils of the SQUID magnetometer for each scan. The system automatically performs a Lorentzian fit of the recorded magnetic signals, relative to the position of the sample inside the holder. The data output by the system is in units of emu. However, because the sample holder used is often diamagnetic, it is important to calibrate the sample stage without the sample to remove this additive factor as well as any temperature dependence associated with the diamagnetism. In Fig. 4.3(a), normalized magnetization data for LCMO and LCCO are obtained using the SQUID system, where $T_{\text {Curie }} \approx 260 \mathrm{~K}$ and $180 \mathrm{~K}$ for LCMO and LCCO, respectively, are verified.

To measure the ac susceptibility, a local Hall probe magnetometer is used. It is placed in the center of an excitation coil, so when a sample is placed directly on top of the Hall probe, its measurement of the local magnetic induction corresponds to the response 
of the sample to the applied ac magnetic field from the coil. In order for this technique to work, it is important that the size of the sample is much larger than the active region of the Hall probe to ensure that no excess stray field passing around the sample would be picked up by the magnetometer. The physical quantity measured by the Hall probe is the magnetic flux that penetrates through the sample, or the transmittivity $T_{H}$. This parameter is directly related to the susceptibility by the relation $T_{H}=1+\chi$ (in SI units). To perform the ac measurement, a rather simple lock-in technique is employed. Using a lock-in amplifier, its built-in signal generator can be connected to the excitation coil directly, with the output of the Hall probe connected to the lock-in amplifier in differential mode. Only an additional dc current source is needed to drive the Hall probe. In Fig. 4.3(b), the real part of the ac magnetic susceptibility $\chi^{\prime}$ for LCMO is presented.

\subsection{Results}

The lattice distortion induced by the substrates yields two relevant effects. One is the lattice strain, defined as $\Delta a_{0} / a_{0}$, where $a_{0}$ is the lattice constant of the bulk perovskite, and $\Delta a_{0}$ is the difference between the lattice constant of the film and that of the bulk. The other is the lattice relaxation between the substrate and the film, defined as $\Delta a_{s} / a_{s}$, where $a_{s}$ is the lattice constant of the substrate, and $\Delta a_{s}$ is the difference between the lattice constant of the film and that of the substrate. For films thicker than a critical thickness, the epitaxial films may acquire lattice constants different from those of the substrates, thereby giving rise to extrinsic distortions such as dislocations, grain boundaries,

and domains. Lattice strain gives rise to more intrinsic distortion such as variation of the 
magnetic exchange and electron-phonon interactions. These two types of lattice distortion induced by three different substrates are quantified by a Caltech undergraduate student Geoff Beach in his senior thesis using high-resolution x-ray diffraction studies and are and listed in Table 4.1 for the LCMO and LCCO films. Among the LCMO films, the lattice strain for the $a$ - and $b$-axes is the largest in LCMO/STO, and the lattice relaxation is the largest in LCMO/YAO. On the other hand, significant distortion in the LCCO films occurs only in the lattice relaxation for LCCO/YAO.

\begin{tabular}{|c|c|c|c|c|c|}
\hline Compound & Lattice & Relaxation (\%) & Lattice & Strain & $(\%)$ \\
\hline & $\frac{\Delta a_{s}}{a_{s}}$ & $\frac{\Delta b_{s}}{b_{s}}$ & $\frac{\Delta a_{0}}{a_{0}}$ & $\frac{\Delta b_{0}}{b_{0}}$ & $\frac{\Delta c_{0}}{c_{0}}$ \\
\hline $\mathrm{LCMO} / \mathrm{LAO}$ & 1.32 & 1.64 & 0.05 & -0.93 & 1.58 \\
\hline $\mathrm{LCMO} / \mathrm{YAO}$ & 4.80 & 3.08 & 0.57 & -0.10 & 1.01 \\
\hline $\mathrm{LCMO} / \mathrm{STO}$ & 0.62 & 0.56 & 1.07 & 0.95 & -0.39 \\
\hline $\mathrm{LCCO} / \mathrm{LAO}$ & -0.05 & -0.05 & -0.18 & -0.18 & -0.11 \\
\hline $\mathrm{LCCO} / \mathrm{YAO}$ & 4.59 & 1.41 & 0.82 & 0.68 & -0.53 \\
\hline
\end{tabular}

Table 4.1: The lattice constants, lattice relaxation, and lattice strain determined from x-ray diffraction for $\mathrm{La}_{0.7} \mathrm{Ca}_{0.3} \mathrm{MnO}_{3}(\mathrm{LCMO})$ and $\mathrm{La}_{0.5} \mathrm{Ca}_{0.5} \mathrm{CoO}_{3}$ (LCCO) epitaxial films on $\mathrm{LaAlO}_{3}(\mathrm{LAO}), \mathrm{YAlO}_{3}(\mathrm{YAO})$ and $\mathrm{SrTiO}_{3}$ (STO) substrates at $300 \mathrm{~K}$.

The effects of lattice distortion on the resistivity and magnetoresistance of the LCMO films are illustrated in Fig 4.4(a)-(c), showing the highest zero-field resistivity, $\rho(H=$ 0 ), and the largest magnetoresistance, $\Delta R_{H}$, at $H=60 \mathrm{kOe}$ in the LCMO/YAO film, which has the largest lattice relaxation. Here the magnetoresistance in a magnetic field $H$ is defined as $\Delta R_{H} \equiv[\rho(0)-\rho(H)] / \rho(H)$. Attributing the various physical properties to the substrate-induced lattice distortion is supported by the optical studies of the same LCMO films [106], which show distinct substrate-dependent frequency shifts $\left(\sim 10 \mathrm{~cm}^{-1}\right)$ in the transverse optical phonon modes associated with the Mn-O-Mn bending $\left(\sim 330 \mathrm{~cm}^{-1}\right)$ and 
Mn-O stretching $\left(\sim 580 \mathrm{~cm}^{-1}\right)$ modes $[106,107]$. The frequency shifts increase with the increasing lattice strain $\Delta a_{0} / a_{0}$, and these unusually large frequency shifts with the lattice strain suggest strong electron-phonon coupling [107], and are also supportive of the lattice polaron conduction scenario $[88,96,98]$. For the least distorted films on the LAO substrate, the optical phonon frequencies are in good agreement with those in polycrystalline samples [107]. It is worth noting that the linewidths of these optical phonon modes are found to be consistently narrower in thin films than those in polycrystalline samples.

In Fig 4.5(a)-(c), resistivity data as functions of the magnetic field at various constant temperatures are shown for the annealed LCMO/LAO, LCMO/YAO, and LCMO/STO films. The $\rho-H$ isotherms are monotonically decreasing with $H$ for all samples at all temperatures, and those for the least distorted LCMO/LAO films are the smoothest. In contrast, for both LCMO/STO and LCMO/YAO films, some isotherms exhibit a distinct change in the slope.

In Fig. 4.6(a) and (b), the resistivity and magnetoresistance data as a function of temperature are shown for the LCCO/LAO and LCCO/YAO films. The result for LCCO/STO is omitted as the result of poor quality of the film because of interdiffusion with the substrate material. The resistivity of these LCCO films are notably more conducting than their LCMO counterparts, and in the place of the resistivity peaks near the Curie temperature $T_{\text {Curie }}$, "kinks" in the resistivity curves are observed instead. Also, the corresponding negative magnetoresistance measures only a few percent change in $H=60$ $\mathrm{kOe}$. 

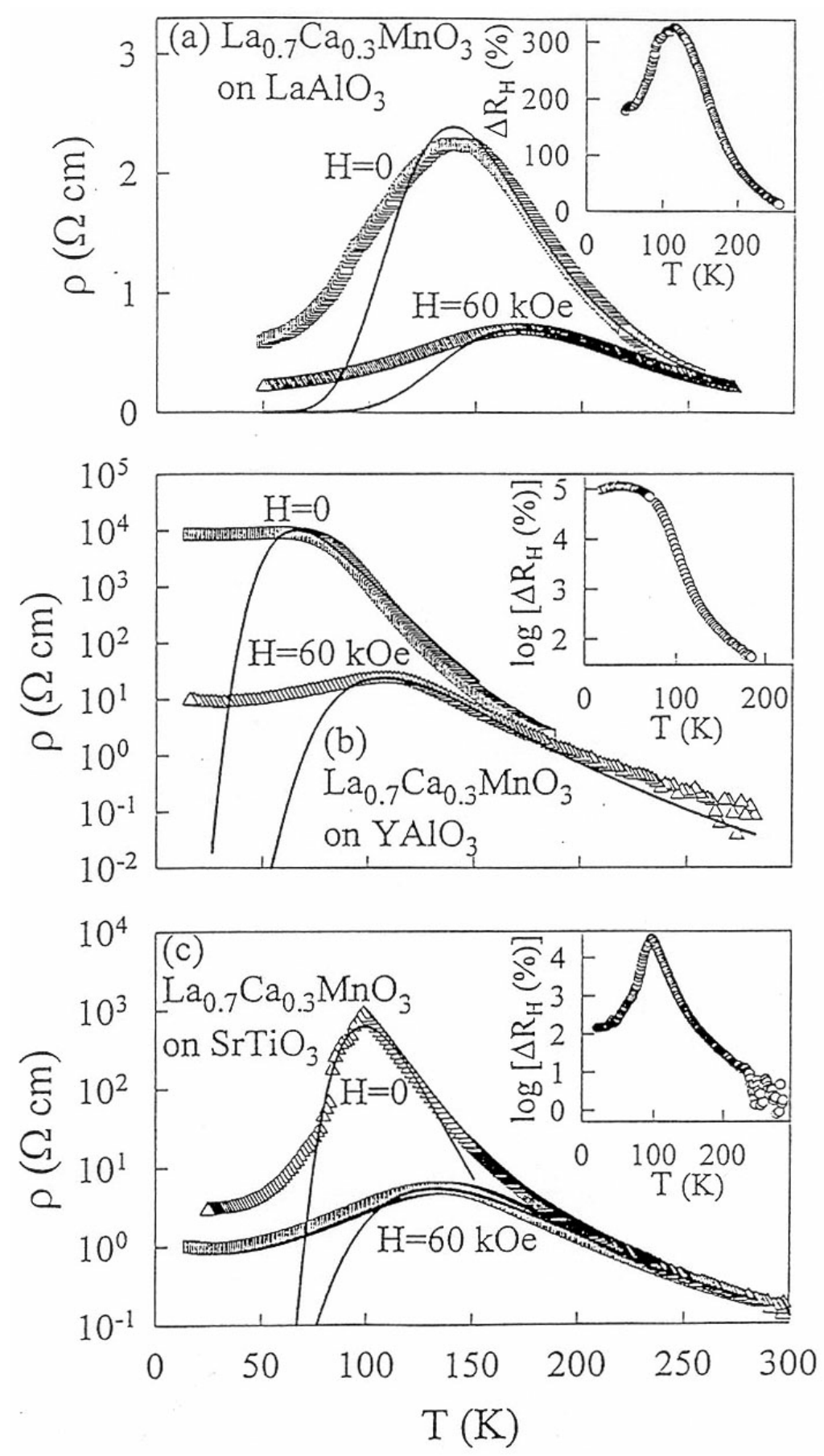

Figure 4.4: The effect of lattice distortion on the resistivity of $\mathrm{La}_{0.7} \mathrm{Ca}_{0.3} \mathrm{MnO}_{3}$ epitaxial films on different substrates: a) $\mathrm{LaAlO}_{3}$, b) $\mathrm{YAlO}_{3}$; c) $\mathrm{SrTiO}_{3}$. The corresponding magnetoresistance $\left(\Delta R_{H}\right)$ versus temperature $(T)$ data are shown in the insets [58]. 

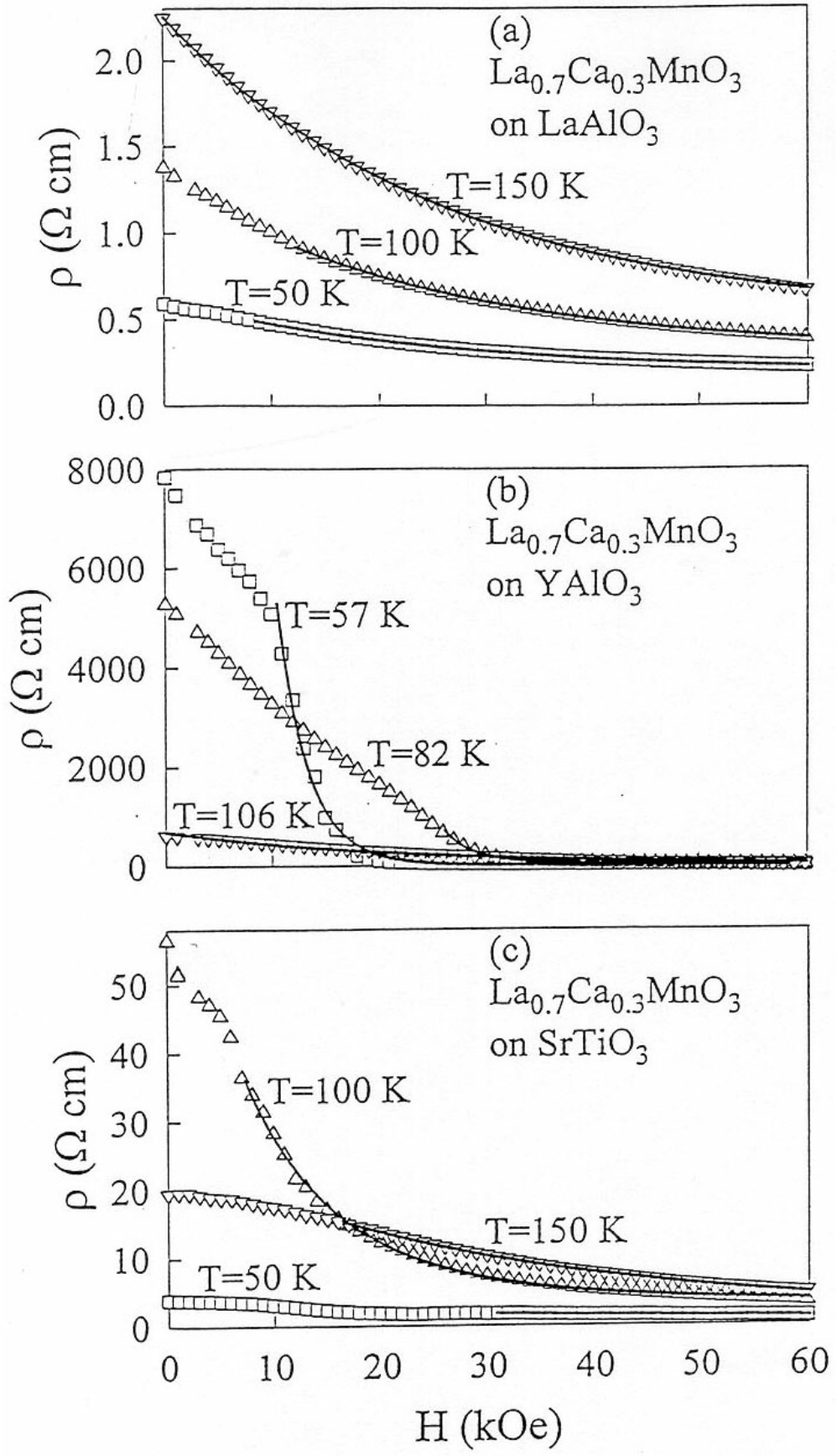

Figure 4.5: The magnetic field dependence of the resistivity at various constant temperatures for a) LCMO/LAO, b)LCMO/YAO, and c) LCMO/STO epitaxial films [58]. 

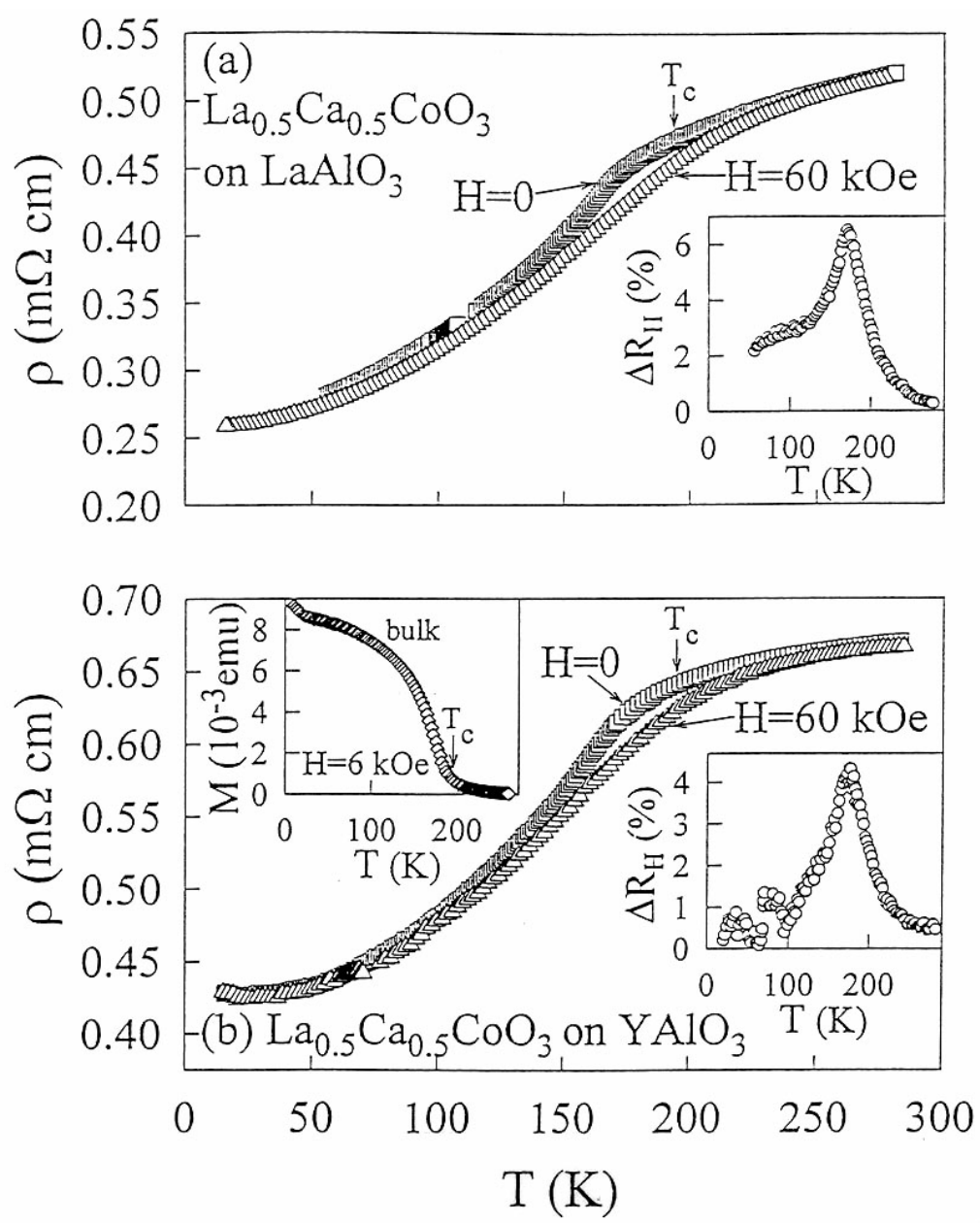

Figure 4.6: The effect of lattice distortion on the resistivity of $\mathrm{La}_{0.5} \mathrm{Ca}_{0.5} \mathrm{CoO}_{3}$ epitaxial films on different substrates: a) $\mathrm{LaAlO}_{3}$; b) $\mathrm{YAlO}_{3}$. The corresponding magnetoresistance $\left(\Delta R_{H}\right)$ versus temperature $(T)$ data as well as $M-T$ cirve are shown in the insets [58]. 


\subsection{Analyses}

The lattice polaron conduction mechanism has been considered as important in LCMO films at high temperatures $\left(T \gtrsim T_{\text {Curie }}\right)$ because the hopping rate of the itinerant electrons in the semiconducting state is sufficiently slow and comparable to the optical phonon frequency. Assuming dominant polaron conduction at high temperature $(T \sim$ $\left.T_{\text {Curie }}\right)$, the first-order polynomial approximations for the polaron conductivity are $[98,108]$ :

$$
\begin{gathered}
\rho(T) \approx \frac{\alpha T}{1+G} \exp \left[\frac{E_{b}(T)}{k_{B} T}\right] \equiv \frac{\alpha T}{1+G} \exp \left\{\frac{E_{b 0}}{k_{B} T}[1-G(T)]\right\} \\
\rho(H) \approx \frac{\alpha T}{1+G^{\prime}} \exp \left[\frac{E_{b}(H)}{k_{B} T}\right] \equiv \frac{\alpha T}{1+G^{\prime}} \exp \left\{\frac{E_{b 0}}{k_{B} T}\left[1-G^{\prime}(H)\right]\right\}
\end{gathered}
$$

where $E_{b}$ is the polaron binding energy, $\alpha$ is a constant, and the unknown temperature and magnetic field dependences of $E_{b}$ are approximated by $G(T)$ and $G^{\prime}(H)$, respectively, which should satisfy the conditions imposed by the polaron model. More specifically, $E_{b} \rightarrow 0$ in the limit of complete magnetic order when the increasing hopping rate of the itinerant electrons exceeds the optical phonon frequency, and $E_{b} \rightarrow$ constant in the absence of longrange magnetic order. Thus, $0 \leq G, G^{\prime} \leq 1$, and $G(T) \rightarrow 1$ for $T \ll T_{\text {Curie }}, G^{\prime}(H) \rightarrow 1$ for large $H$. Furthermore, $G(T) \rightarrow 0$ for $T \rightarrow T_{C u r i e}$, and $G^{\prime}(H) \rightarrow 0$ for $H \rightarrow 0$. Using the polaron conduction equations and the constraints given above, the best fitting curves are obtained and shown as the solid lines in Figs. 4.4(a)-(c) for the high-temperature $\rho-T$ data (for $T$ near and above the resistive peaks) and and in Figs. 4.5(a)-(c) for the high field $\rho-H$ data (for $H$ larger than where the "kink" in the $\rho(H)$ curves appear), with the same parameter $E_{b 0} \approx 0.35 \mathrm{eV}$ for all LCMO films on different substrates. This yields empirical $G(T)$ and $G^{\prime}(H)$ functions illustrated in Fig 4.7(a) and (b). On the 
other hand, the deviation of the resistivity data from the polaron model increases with the increasing substrate-induced lattice distortion at low temperatures, suggesting that the residual resistivity and magnetoresistance of those samples are largely determined by the extrinsic lattice distortions such as domain walls and grain boundaries.

A noteworthy correlation of the functions $G(T)$ and $G^{\prime}(H)$ with the normalized magnetization $m \equiv M / M_{s}$, where $M$ is the magnetization and $M_{s}$ is the saturation magnetization is illustrated in Fig. 4.7(a) and (b). Here, the unnormalized experimental magnetization data $M(T)$ and $M(H)$ are taken using a SQUID magnetometer and are plotted in the insets in Fig. 4.7(a) and (b). This correlation suggests the relevance of magnetic ordering to the electrical conduction, particularly for temperatures near $T_{\text {Curie }}$. The derived polaron binding energy $\left(E_{b 0} \approx 0.35 \mathrm{eV}\right)$ compares favorably with the Jahn-Teller energy of $\sim 0.5 \mathrm{eV}$ for undoped $\mathrm{LaMnO}_{3}[88,96]$, and is much larger than that for the magnetic polarons due to the electron-spin interaction [109]. Since the equations given in the polaron model are only approximate and limited to the high-temperature region, the exact forms of $G(T)$ and $G^{\prime}(H)$ should not be taken literally. However, the analyses do provide a consistent picture of two types of contribution to the resistivity and magnetoresistance, with strong evidence for polaron hopping conduction at high temperatures, and a different scattering mechanism associated with the lattice distortion at low temperatures.

The low-temperature scattering mechanism may be understood by comparing $M(T)$ data for all LCMO films and that for the bulk in Fig. 4.7(a). The slower rise of magnetization below $T_{\text {Curie }}$ for samples of larger lattice distortion, either intrinsic (strain) or extrinsic (relaxation), appears to be correlated with larger resistivity and magnetoresis- 

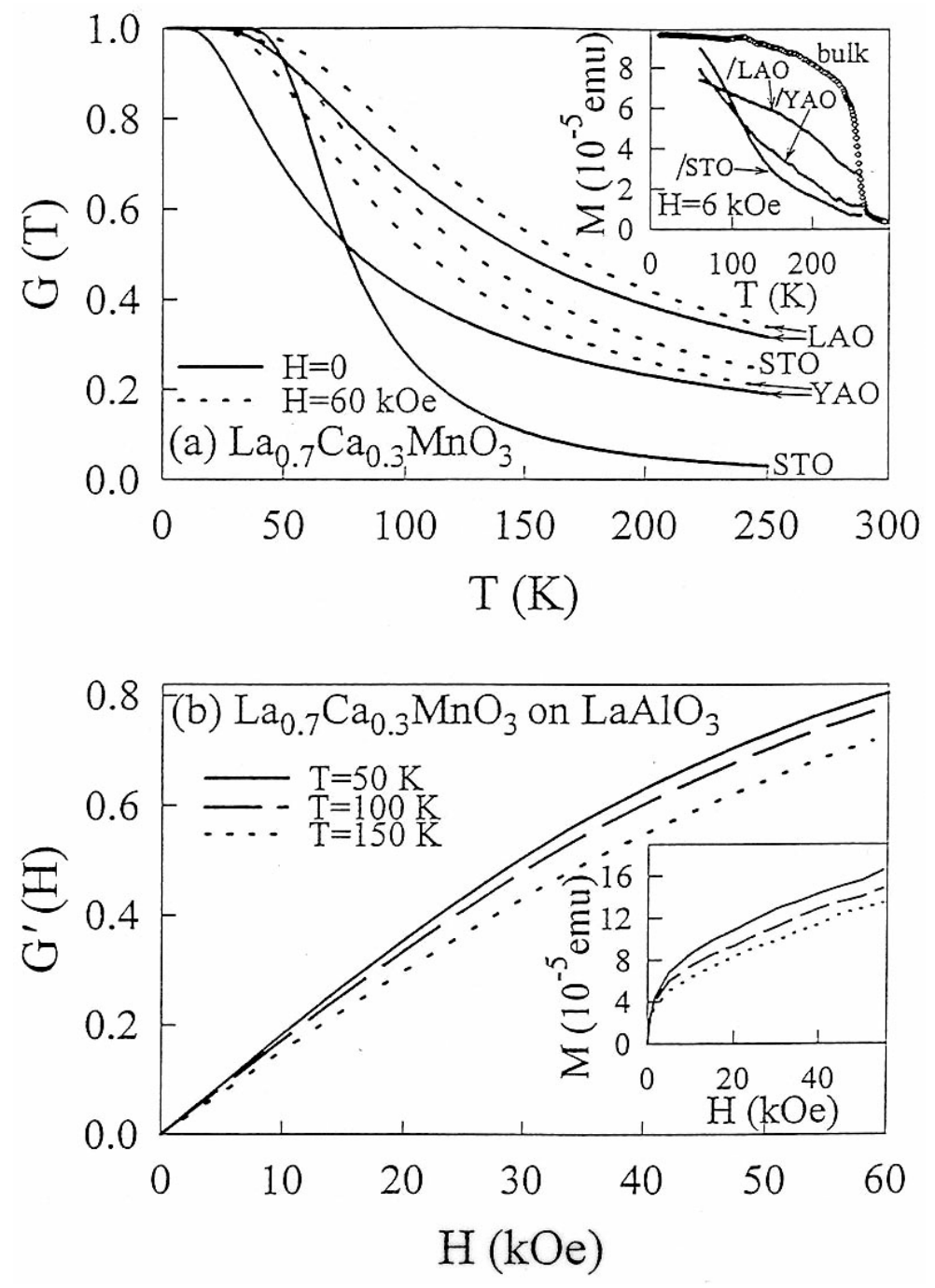

Figure 4.7: a) The $G(T)-T$ curves for LCMO/LAO, LCMO/STO, and LCMO/YAO films (solid lines) at $H=0$ and $H=60 \mathrm{kOe}$. The inset shows the temperature dependence of the magnetic moments $M(T)$ for LCMO/LAO, LCMO/STO, and LCMO/YAO films, and bulk LCMO, taken at $H=6 \mathrm{kOe}$. b) The representative $G^{\prime}(H)-H$ isotherms for the LCMO/LAO film. The corresponding $M-H$ data are shown in the inset [58]. 
tance, suggesting increasing electron scattering induced by larger lattice distortion. One possible consequence of larger lattice distortion is a larger number of magnetic domains. Although all domains may undergo a ferromagnetic phase transition at the same $T_{C u r i e}$, the incomplete alignment of the moments of the magnetic domains resulting from inhomogeneity or pinning by local defects below $T_{\text {Curie }}$ gives rise to slower rising magnetization and larger scattering of conduction electrons. Therefore, an applied magnetic field has more significant effect on reducing the resistivity in samples with larger lattice distortion through the aligning of the magnetic domains [53].

The picture of magnetic domain wall scattering is consistent with the larger resistivity and magnetoresistance at low temperatures where the polaron contribution becomes insignificant. The distinct change of slope in low-temperature $\rho-H$ isotherms of $\mathrm{LCMO} / \mathrm{STO}$ and LCMO/YAO, which are samples with larger lattice distortion, also suggests better alignment of magnetic domains in sufficiently high fields. In contrast, the $\rho-H$ isotherms of the least distorted LCMO/LAO can be described by the polaron conduction equation over a large magnetic field range, consistent with less significant magnetic domain wall scattering [53].

In the $\mathrm{La}_{0.5} \mathrm{Ca}_{0.5} \mathrm{CoO}_{3}$ epitaxial films, despite comparable lattice relaxation and lattice strain to the manganites in LCCO/YAO, the magnitude and temperature dependence of the resistivity in the LCMO and LCCO systems exhibit sharp contrasts. Since the higher electron mobility in the cobaltites tends to inhibit the formation of lattice polarons, the resistivity of the cobaltites may be understood in terms of the combination of conventional impurity, phonon and disorder-spin scattering, with the disorder-spin scattering being the 
only magnetic field-dependent term. For both LCCO/LAO and LCCO/YAO samples, a faster decrease in the zero-field resistivity occurs below the Curie temperature $T_{\text {Curie }} \approx 180$ $\mathrm{K}$, indicating that the magnetic ordering below $T_{\text {Curie }}$ reduces the resistivity and that the small negative magnetoresistance near $T_{C u r i e}$ is due to the field-induced suppression of spin fluctuations and of the corresponding scattering near $T_{C u r i e}$. It appears that the physical origin of the negative magnetoresistance in the cobaltites is fundamentally different from that in the manganites, and the formation of lattice polarons seems to be essential for the occurrence of CMR effects in the manganites. However, recent STM studies [110] and numerical simulations $[111,112]$ have shown that for certain range of doping levels, a firstorder phase transition can take place either at $T_{C u r i e}$ or under a large applied magnetic field, and that nanoscale inhomogeneities associated with competing phases of ferromagnetism and antiferromagnetism can occur. This scenario complements the lattice polaron model that restricts to high temperatures and provides feasible account for the occurrence of first-order phase transitions in certain manganites and for the conductivity over a wide temperature range.

Finally, it should be remarked that the cobaltites $\mathrm{La}_{1-\mathrm{x}} \mathrm{M}_{\mathrm{x}} \mathrm{CoO}_{3}(\mathrm{M}=\mathrm{Ca}, \mathrm{Sr})$ also exhibit very interesting and unique physical properties. In particular, giant ferromagnetic Hall effect with interesting doping and temperature dependence has been discovered in studies from this group $[113,114]$. A record ferromagnetic Hall coefficient among all single-phase ferromagnets is found in $\mathrm{La}_{0.8} \mathrm{Ca}_{0.2} \mathrm{CoO}_{3}$, which has the doping composition near the magnetic percolation threshold [113]. However, those results are not directly related to the spin-injection studies, so will not be discussed further in this thesis. 


\section{Chapter 5}

\section{Spin-injection Experiment}

\subsection{Sample Characterization}

Having discussed details of the fabrication conditions in Sec. 3.1 and the properties of the manganites, the attention can now be shifted to the spin injection experiments. It should be emphasized that the close lattice match among the chosen constituent layers of the perovskite F-I-S and the substrates facilitated epitaxial film growth [51], thus minimizing strong spin-flip scattering at the interface and preserving spin polarization during injection. For electrical contact, each of the YBCO and STO (LNO) layers had four gold pads placed on top using sputtering deposition, as illustrated in Fig. 5.1. The compositional quality of these heterostructures were examined using X-ray photoelectron spectroscopy (XPS) [53]. To ensure no discernible reaction between layers during the growth process, XPS studies of bi-layers of YBCO/STO and STO/LSMO on LAO were monitored by Dr. Vasquez at JPL and the absence of reaction within $\sim 0.1$ atomic percent for at least the top $10 \mathrm{~nm}$ of the YBCO layer was confirmed. 


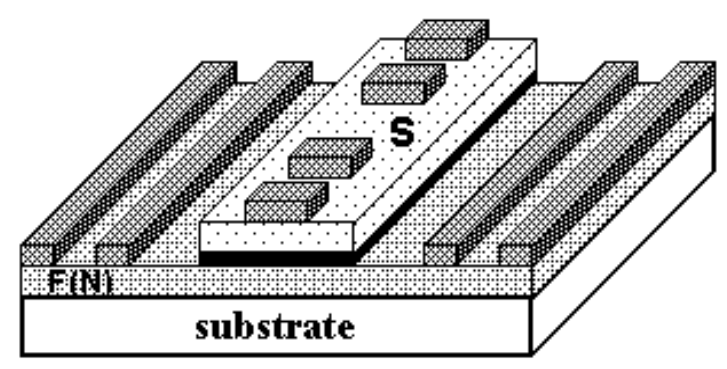

Figure 5.1: As-grown heterostructure with gold contacts deposited.

To verify the quality of samples, electrical transport measurements were performed on both the superconducting and ferromagnetic layers to determine the normal-state resistivity $\rho_{n}$ and the transition temperatures $T_{c}$ and $T_{\text {Curie }}$ (see Fig. 5.2). In addition, scanning tunneling spectroscopy was also performed on the YBCO layer of the F-I-S and N-I-S samples, and the superconducting gap value was found to be consistent with that of the optimally doped YBCO single crystals $[54,55]$. The LAO substrate was chosen because it had been demonstrated to be the substrate that yielded minimum lattice strain and the best magnetization alignment for the thin-film growth of manganites $[58,59,106]$, as explained in Chapter 4. Our characterizations revealed that the resistivity of each constituent layer of the heterostructures and the $T_{C u r i e}$ of the ferromagnetic manganites were all comparable to those of the corresponding single crystalline materials [53]. Since the resistivity of the manganite is known to couple strongly to the magnetic properties and therefore is a characterization for the quality of the manganite as detailed in Chapter 4, a manganite layer with resistivity comparable to that of a single crystal implies large and relatively well-aligned ferromagnetic domains $[58,59,60,106]$. The temperature dependence of the resistivity in the manganite layer always exhibited either a maximum or a distinct change in 


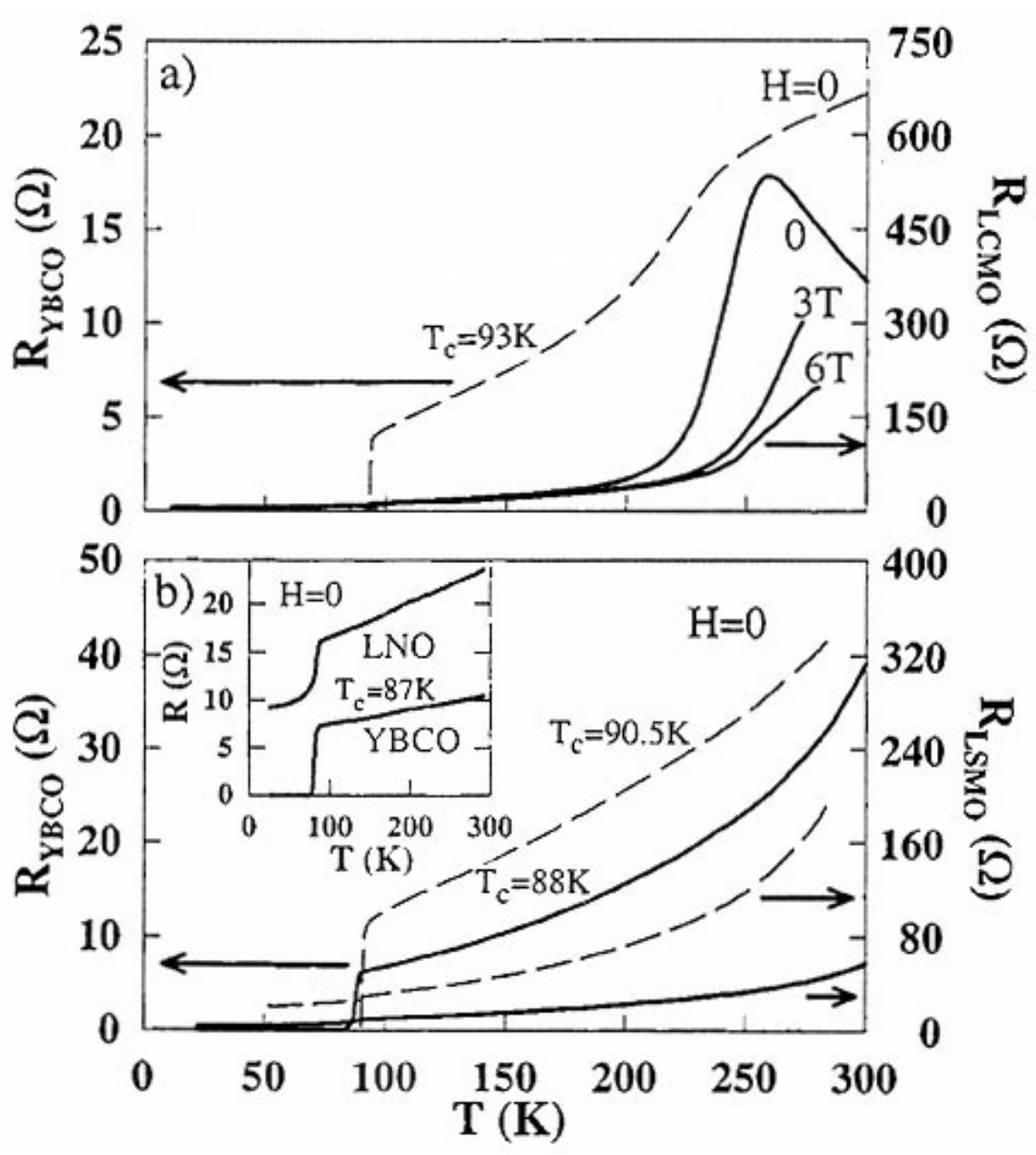

Figure 5.2: a) Resistance $(R)$ vs. temperature $(T)$ curves of the YBCO (left axis) and LCMO (right axis) in the YBCO/YSZ/LCMO (100/1.3/100 nm) heterostructure. b) The $R$ vs. $T$ curves of the YBCO (left axis) and LSMO (right axis). The solid lines denote the data for YBCO/STO/LSMO $(100 / 2 / 100 \mathrm{~nm})$, and the dashed lines are the data for YBCO/STO/LSMO $(100 / 10 / 100 \mathrm{~nm})$. The inset includes data for YBCO/STO/LNO $(100 / 2 / 100 \mathrm{~nm})[53]$. 
slope near $T_{\text {Curie }}(\sim 260 \mathrm{~K}$ for LCMO and $\sim 320 \mathrm{~K}$ for LSMO), which was characteristic of high-quality ferromagnetic manganites $[58,59,115]$. On the other hand, the superconducting transition temperature $\left(T_{c}\right)$ of $\mathrm{YBCO}$ varied somewhat among devices, ranging from 84 to $90 \mathrm{~K}$, with no apparent correlation with the YBCO thickness. The $T_{c}$ variation is attributed to uncertainties in the substrate temperature during the thin film growth. Due to the variation in $T_{c}$, the temperature dependence of various physical quantities of YBCO shall be considered in reduced temperature $\left(T / T_{c}\right)$ rather than absolute temperature $T$.

\subsection{Prevention of Artifacts from Joule Heating}

One technical issue that arises during dc injection/critical current measurements is the possibility of Joule heating. In the current injection experiment, it is necessary to apply currents comparable to the critical current of the YBCO layer (sometimes on the order of several hundred milliamperes depending on the reduced temperature). Passing current of that magnitude will generate a non-negligible amount of heat, which, if not properly dissipated away, will undoubtedly raise the temperature of the sample. Proper heat sinking in this case is difficult to achieve as the resistive CMR layer essentially becomes a perfect heater to the superconducting layer because of its proximity. In addition, the same current passing through the serial contact resistances inherent to the setup can also easily impart several tens of milliwatts of power to the sample. This artificial temperature increase will contribute to a perceived drop in critical current, which is devastating to the accuracy of this experiment.

To quantify the effect of Joule heating from DC injection measurements, a pulse- 


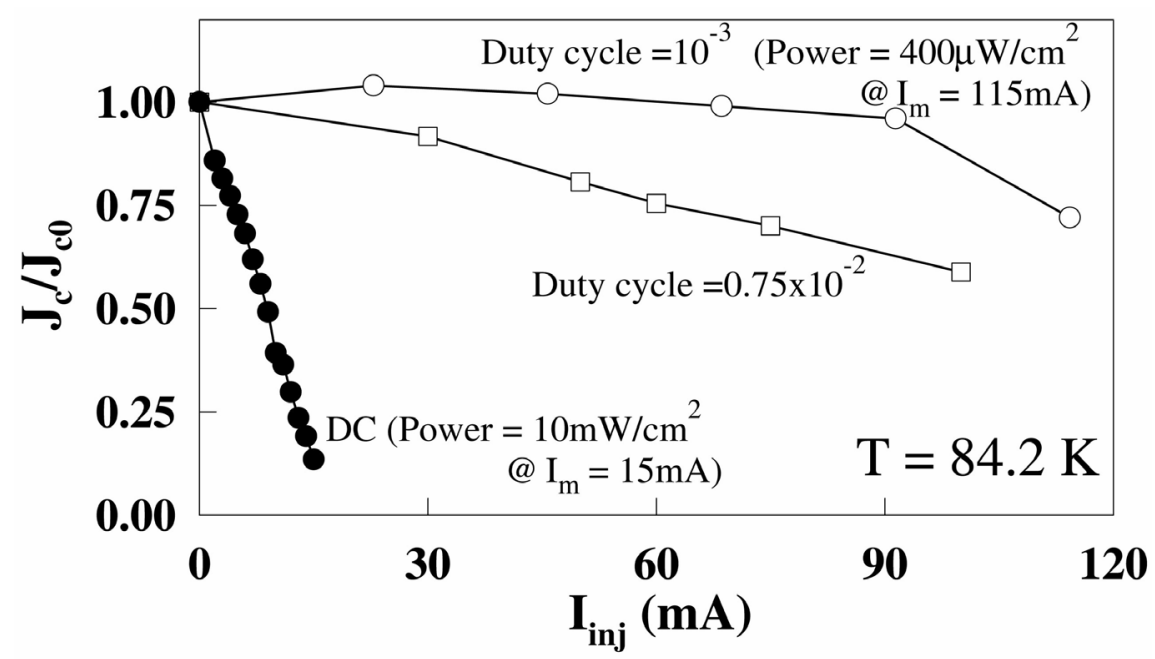

Figure 5.3: Comparison of the normalized critical current density $J_{c} / J_{c 0}$ vs. $I_{i n j}$ of $\mathrm{YBCO} / \mathrm{YSZ} / \mathrm{LCMO}$ for dc and pulse current measurements at $T=84.2 \mathrm{~K}$ [53].

current technique (see following section for details) is employed and used as a comparison. The injection experiment is performed on the same sample using both DC and pulsed currents. Duty cycles of 0.0075 and 0.001 are used and compared with the DC measurement. In Fig. 5.3, the normalized critical current $J_{c} / J_{c 0}$ is plotted versus injection current at $T=84.2 \mathrm{~K}$. While the two pulsed current measurements yielded critical current suppression of $\sim 30-40 \%$ with injection currents near or above $100 \mathrm{~mA}$, the DC current measurement shows nearly complete suppression of critical current with $15 \mathrm{~mA}$ of injection current. Clearly, most of the suppression effect at low injection values in the DC current measurement is the result of Joule heating. 


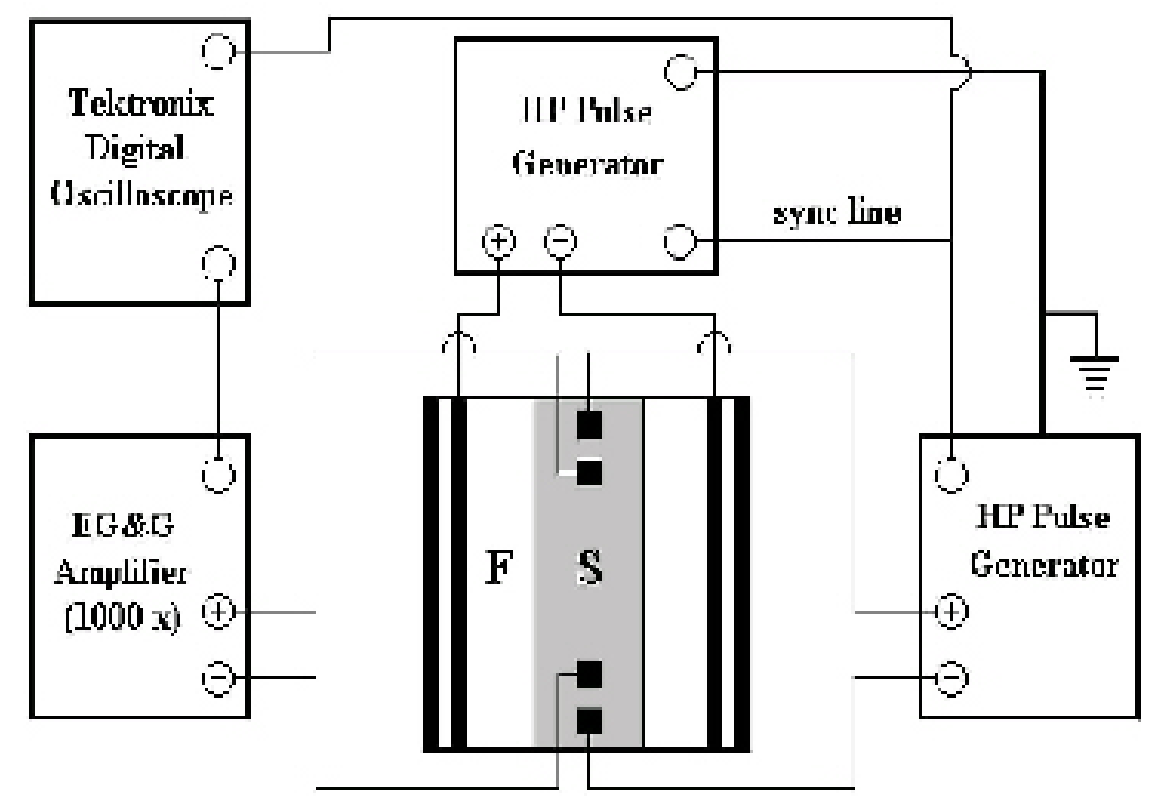

Figure 5.4: Block diagram of the pulse-current technique.

\subsection{Pulse-Current Technique}

In light of the heating effects, the critical current $\left(I_{c}\right)$ measurements of the $\mathrm{F}(\mathrm{N})$ -

I-S heterostructures were made with the pulsed current technique, which synchronized two pulse generators that supplied a measurement current through YBCO and an injection current through the metallic underlayer, reference to a common ground, as illustrated in Fig. 5.4. Here the current sources and nano-voltmeter have been replaced by the two pulse generators and a digital oscilloscope, respectively. That they are voltage sources makes it necessary to monitor the total resistance load of the circuit in the measurement. The full schematic of the measurement is depicted in Fig. 5.5. The advantage of this method was to eliminate undesired Joule heating on the YBCO from power dissipation in the event when high current levels $(<300 \mathrm{~mA})$ passed through the electrical contacts and the resistive 


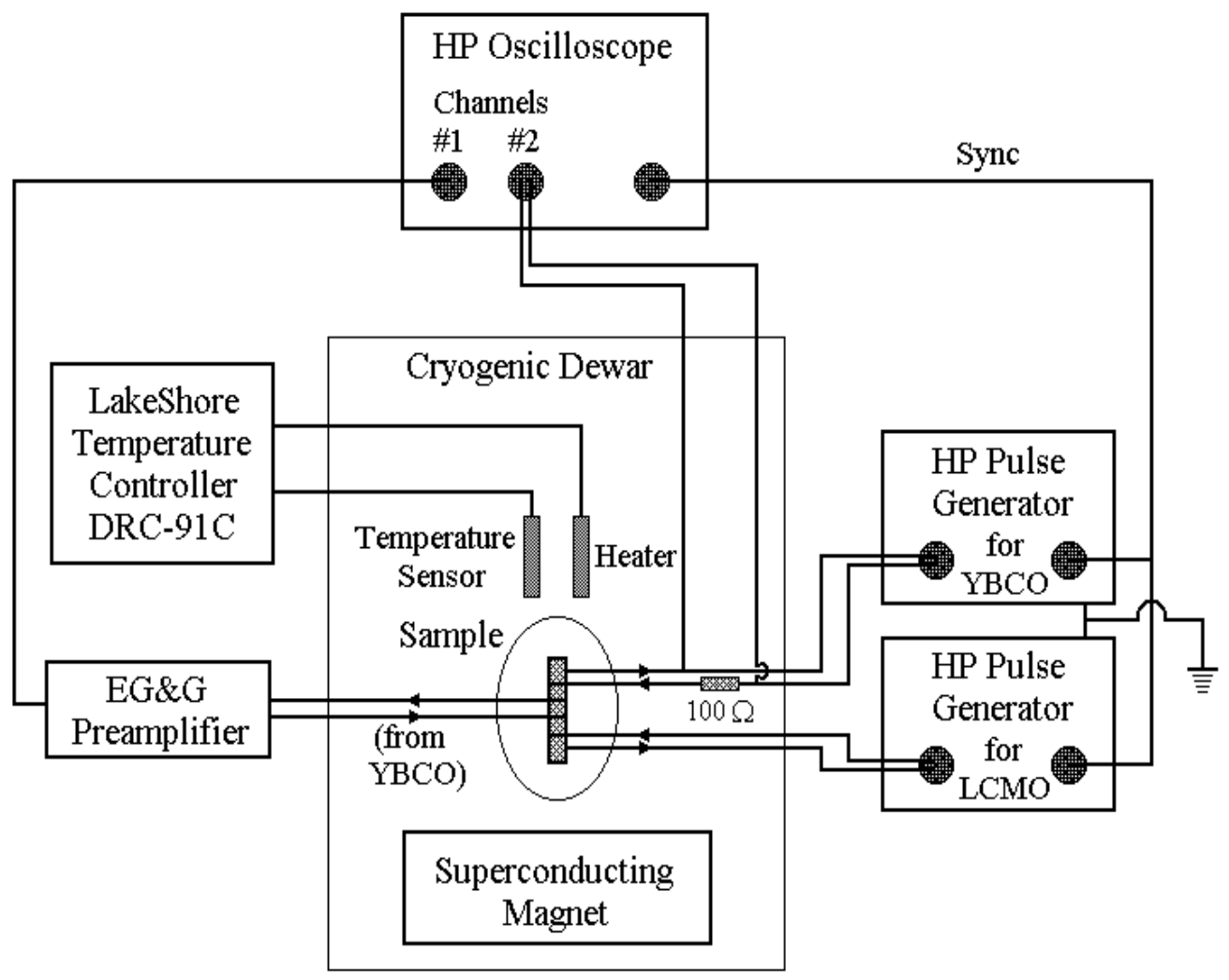

Figure 5.5: Full schematic of the pulse-current measurement, including the cryostat.

metallic underlayer. A 1:1000 ratio of the pulsed current width $\left(t_{w}\right)$ to period $\left(t_{p}\right)$ was chosen, which yielded a negligible temperature increase $(<10 \mathrm{mK})$ in the YBCO during maximum current injection, monitored with in-situ thermometry using the resistivity of the manganite. The pulse width used for this work was $t_{w}=300 \mu \mathrm{s}$.

It is worth noting that the pulsed-current setup employed in our experiment involved the use of pulsed-voltage generators, which linked sources with output impedances comparable to the relevant resistance in the measurement circuit. This setup therefore resulted in a finite, but small, leakage current flow through the pulse generator upon the 
introduction of injection current from the underlayer. However, simple circuit analysis and direct calibration had indicated that the leakage current was less than $10 \%$ of the total injected current for all measurements. In principle, decoupled current paths can be achieved with smaller and lithographically defined devices and with the use of high output-impedance current sources. In that regard, several F-I-S devices with smaller lateral dimensions, ranging from $100 \mu \mathrm{m}$ to $1 \mu \mathrm{m}$, have been fabricated and studied, and the results obtained on those patterned devices will be covered in Chapter 8. However, in this chapter and Chapter 6 , the attention is concentrated on the experimental studies of the larger as-grown devices.

In Fig. 5.6(a), generic I-V curves of an F-I-S heterostructure with zero and a finite injection current are shown. The curve symmetric about the zero-current axis corresponds to the I-V data in the absence of current injection. For a given temperature, the current values that drive the YBCO superconductor to register $+3 \mu \mathrm{V}$ and $-3 \mu \mathrm{V}$ across its voltage terminals $\left(\sim 3 \mathrm{~mm}\right.$ apart) are defined as the critical currents $I_{c}^{+}$and $I_{c}^{-}$, respectively. The second curve to the left shows a shifted I-V curve because of an external injection current $I_{i n j}$ that increases the total current passing through the superconductor. This effect is present for the injection of both simple and spin-polarized quasiparticles. The observed narrowing of the gap in between the $I_{c}^{+}$and $I_{c}^{-}$values with increasing $I_{i n j}$ is the result of critical current suppression due the apparent deterioration of superconductivity from the external perturbation. Therefore, the critical current under quasiparticle injection is defined as $I_{c}=\left(I_{c}^{+}-I_{c}^{-}\right) / 2$, and that in the absence of quasiparticle injection is $I_{c 0}(T)$. The magnitude of the shift in the I-V is related to the amount of current entering the superconductor from the underlayer current injection and is hereafter defined as $I_{i n j}$. Such 
shifts are always present under external injection, as exemplified in Figs. 5.6(a) and (b) for F-I-S and N-I-S samples with comparable thicknesses of YBCO and similar reduced temperatures. The critical current density $J_{c}$ and the injection current density $J_{i n j}$ are obtained by dividing the corresponding currents by the cross section of the superconductor. As shown in Figs. 5.6(a) and (b), the suppression of $J_{c}$ is much more significant in the F-I-S sample. Additional I-V curves for F-I-S heterostructures at other reduced temperatures and for a range of injection currents are shown in Figs. 5.7(a) and (b) for further comparison.

\subsection{Critical Current Suppression}

Besides the effect of injection currents on $J_{c}$, the low-temperature critical current density $J_{c 0}$ in the absence of injection is found to be sensitive to the thickness of the insulator barrier of the F-I-S heterostructures [53], with systematically increasing $J_{c 0}$ for samples with thicker insulating barriers and otherwise identical lateral dimensions, as shown in Fig. 5.8. Similar finding has also been confirmed in our patterned F-I-S devices. Furthermore, the $J_{c 0}$ values of N-I-S samples at low temperatures were larger than the corresponding $J_{c 0}$ of F-I-S samples with the same lateral dimensions and barrier thickness. Such a systematic dependence has ruled out the possibility that self-field induced edge-vortex dissipation might have been the primary cause of $J_{c 0}$ suppression with decreasing insulating barrier, and has been attributed to a "self-injection" phenomenon [53, 117], to be elaborated later in this section.

The first current injection experiment is performed on a $100 \mathrm{~nm}$ thick YBCO sample grown on LSMO with a thin STO barrier thickness of $2 \mathrm{~nm}$ as buffer layer, and 
on LCMO with a $1.3 \mathrm{~nm}$ thick YSZ buffer. Upon injection of spin-polarized current, interestingly, $J_{c}$ exhibits a slight increase with $I_{i n j}$, followed by a pronounced suppression of $J_{c}$ at larger injection currents near $T_{c}$, as shown in Fig. 5.9(a). For comparison, a similar sample on LSMO with $10 \mathrm{~nm}$ thick STO buffer layer and a control sample $\mathrm{YBCO} / \mathrm{STO}(2$ $\mathrm{nm}) / \mathrm{LNO}$ are studied also. In sharp contrast, no visible suppression of $J_{c}$ by $I_{i n j}$ for the thick-barrier F-I-S and for the control sample at all temperatures, as shown in Fig. 5.9(b).

The unintuitive observation of enhancement of $J_{c}$ at low injection of spin-polarized current turns out to be the first empirical manifestation of the "self-injection" phenomenon. Since the predominant $d_{x^{2}-y^{2}}$-wave pairing symmetry of YBCO allows substantial lowenergy excitations, it is expected that a significant population of quasiparticles would already exist in the superconductor even at very low temperatures. Given that impedance mismatch exists at the interface of dissimilar materials for thermal [118] or electrical [46] transport, a chemical potential difference $\delta \mu$ develops if a current flows across the interface. Hence, if an electrical current is applied to the superconductor, and if it shares a common ground with the CMR underlayer, some thermally induced quasiparticles may diffuse from $\mathrm{S}$ to $\mathrm{F}$, yielding $\delta \mu>0$. This will facilitate further quasiparticle diffusion, as depicted in Fig. 5.10(a). Because the ferromagnetic manganites are half metals with nearly perfect spin polarization, only quasiparticles with spins parallel to those of the majority carriers in the ferromagnet may diffuse across the interface, leaving behind spin-polarized quasiparticles in the superconductor. This "self-injection" effect in F-I-S is consistent with the observation of monotonic increase of low-temperature $J_{c}$ with increasing insulator thickness, because increasing interface impedance reduces the transport probability of quasiparticles 
from $\mathrm{S}$ to $\mathrm{F}$ [119]. The quasiparticles present in $\mathrm{S}$ from self-injection possess a net spin polarization opposite to that of the majority carriers in $\mathrm{F}$, thus, by commencing external injection of spin-polarized quasiparticles from the $\mathrm{F}$ underlayer, an initial increase in $J_{c}$ occurs due to the neutralization of the two spin-polarized species, one from self-injected and the other externally injected. However, $J_{c}$ must eventually decrease, as observed in Fig. $5.9(\mathrm{a})$, because increasing $I_{i n j}$ begins to overcome and overwhelm the quasiparticles from self-injection. The external injection is schematically illustrated in Fig. 5.10(b).

In addition to the dependence of critical currents on $J_{i n j}$ and $\left(T / T_{c}\right)$, we have investigated F-I-S and N-I-S samples with different thicknesses of YBCO in order to deduce viable information for a characteristic spin relaxation length $\left(\delta_{s}\right)$. A number of F-I-S devices with different YBCO thicknesses $(d=40 \mathrm{~nm}, 50 \mathrm{~nm}, 100 \mathrm{~nm}$ and $160 \mathrm{~nm})$ together with their corresponding N-I-S control samples $(d=50 \mathrm{~nm}$ and $100 \mathrm{~nm})$ have been fabricated and studied. We note that the $J_{c 0}$ values at $4.2 \mathrm{~K}$ were not a monotonic function of $d$, with $J_{c 0}=5.8 \times 10^{4} \mathrm{~A} / \mathrm{cm}^{2}$ for $d=40 \mathrm{~nm}, 5.2 \times 10^{4} \mathrm{~A} / \mathrm{cm}^{2}$ for $d=50 \mathrm{~nm}, 7.0 \times 10^{4} \mathrm{~A} / \mathrm{cm}^{2}$ for $d=100 \mathrm{~nm}$, and $1.5 \times 10^{4} \mathrm{~A} / \mathrm{cm}^{2}$ for $d=160 \mathrm{~nm}$. Detailed current-injection effects on these F-I-S and N-I-S samples are described in the following section.

The critical current density $\left(J_{c}\right)$ provides a macroscopic measure that empirically characterizes the effect of quasiparticle injection on superconductivity. Given a constant thickness of the insulating barrier and the same lateral dimensions of the superconductor, $J_{c}$ is determined by the temperature $(T)$, the injection current density $\left(J_{i n j}\right)$, the characteristic sample dimension $(d)$ along the direction of quasiparticle injection, and the microscopic mechanism for quasiparticle transport across the interface and interaction in the supercon- 
ductor. The dependence of $J_{c}$ on the YBCO thickness is the result of a finite quasiparticle relaxation length along the $c$-axis of the superconductor if all other parameters are kept the same. Through this dependence, the $c$-axis spin-polarized and simple quasiparticle relaxation lengths can be estimated by studying F-I-S and N-I-S with a range of different YBCO thickness.

Two sets of representative $J_{c^{-} \text {-vs.- }} J_{i n j}$ isotherms taken on F-I-S heterostructures with $d=40$ and $160 \mathrm{~nm}$ are shown in Figs. 5.11(a) and (b), respectively. We found that nearly full suppression of critical current could be achieved at lower reduced temperatures in the F-I-S with a thinner $(40 \mathrm{~nm})$ YBCO than that with a thicker $(160 \mathrm{~nm})$ YBCO FI-S heterostructure, with the latter only beginning to exhibit discernible suppression due to current injection above the reduced temperature $>0.97$. This result is consistent with the notion of a finite $c$-axis spin-polarized quasiparticle relaxation length. That is, the manifestation of nearly complete critical current suppression should correlate closely with a $c$-axis spin relaxation length $\delta_{s}^{c}(T)$ approaching the YBCO thickness $(d)$. Thus, spinpolarized quasiparticles are expected to survive throughout nearly the entire thickness of YBCO when strong $J_{c}$ suppression is observed. Under this premise, studies of the $J_{c}$-vs.- $J_{i n j}$ isotherms for F-I-S samples with different YBCO thicknesses can provide a viable measure for the temperature dependence of the $c$-axis spin relaxation length. In contrast, the relative ratio of critical current suppression by a finite $J_{i n j}$ at a given $\left(T / T_{c}\right)$ was appreciably smaller in the N-I-S samples, as shown in Refs. $[53,54]$ where no discernible $J_{c}$ suppression could be detected in an N-I-S sample with a YBCO thickness $d=100 \mathrm{~nm}$. It is necessary to point out that the thickness dependence of spin injection effects cannot be attributed to 
better superconductivity in thicker YBCO. In fact, the $J_{c 0}$ value of the sample with the largest YBCO thickness turns out to be the smallest among all samples at $4.2 \mathrm{~K}$.

\subsection{Efficiency}

A useful definition for experimental characterization of our devices, in normalized current densities, is given by

$$
\eta\left(T, J_{i n j}\right) \equiv \frac{\Delta J_{c}\left(T, J_{i n j}\right)}{J_{i n j}} \equiv \frac{\left[J_{c 0}(T)-J_{c}\left(T, J_{i n j}\right)\right]}{J_{i n j}}
$$

where $\eta$ is defined as the efficiency of quasiparticle injection that relates the magnitude of critical current density suppression to a given amount of injection current density. The temperature and injection current dependence of the efficiency for the F-I-S and N-I-S heterostructures with the same YBCO thickness can provide insightful comparison for the spin and charge transport in the cuprate superconductors.

In addition to the strong dependence of the $J_{c^{\text {-vs.- }}} J_{i n j}$ behavior on the thickness of F-I-S heterostructures, we also compared the efficiencies $\eta\left(T, J_{i n j}\right)$ in F-I-S and N-I-S samples, which were considered to better quantify the suppression of $J_{c}$ due to nonequilibrium quasiparticle injection. Our definition of the efficiency in Eq. (5.1) is equivalent to the definition of a "gain" in the devices by others [51, 52]. As shown in Fig. 5.12(a) and (b), a distinct contrast was observed between the isotherms of the efficiency in F-I-S $\left(\eta_{s}\right)$ and those in N-I-S $\left(\eta_{n}\right)$ devices with comparable YBCO thickness as a function of $J_{i n j}$. In general, $\eta_{s}$ in F-I-S was significantly larger than the corresponding $\eta_{n}$ in N-I-S for all reduced temperatures. Evidently, for F-I-S devices with $d \leq \delta_{s}^{c}(T)$, an anomalous strong decrease in $\eta_{s}$ with increasing $J_{i n j}$ was found only in F-I-S samples at low temperatures. 
Furthermore, for reduced temperatures $0.16 \leq\left(T / T_{c}\right) \leq 0.31$ in these F-I-S devices, $\eta_{s}$ exhibited a non-monotonic dependence with $J_{i n j}$, and then became monotonically increasing with $J_{i n j}$ for $0.31<\left(T / T_{c}\right)<1$. Interestingly, we note that at low spin-polarized quasiparticle injections, the "gain" was actually greater than unity. In contrast, $\eta_{n}$ for the control N-I-S devices appeared to increase monotonically with $J_{i n j}$ at all temperatures, and the magnitude of $\eta_{n}$ was always much smaller than unity.

We also remark that the application of an external magnetic field to the F-I-S devices does not result in appreciable differences in the spin injection effects. That is, the ratio of critical current suppression due to spin injection under a finite magnetic field, given by $\left[J_{c}(H, T, 0)-J_{c}\left(H, T, J_{i n j}\right)\right] / J_{c}(H, T, 0)$, is comparable to that in the absence of magnetic field. This finding may be attributed to the fact that the average dimension of the ferromagnetic domains (typically several microns) in the manganite layer is much larger than the in-plane superconducting coherence length of YBCO, so that further alignment of the magnetic domains with an external field does not result in discernible changes in the ratio of critical current suppression. 

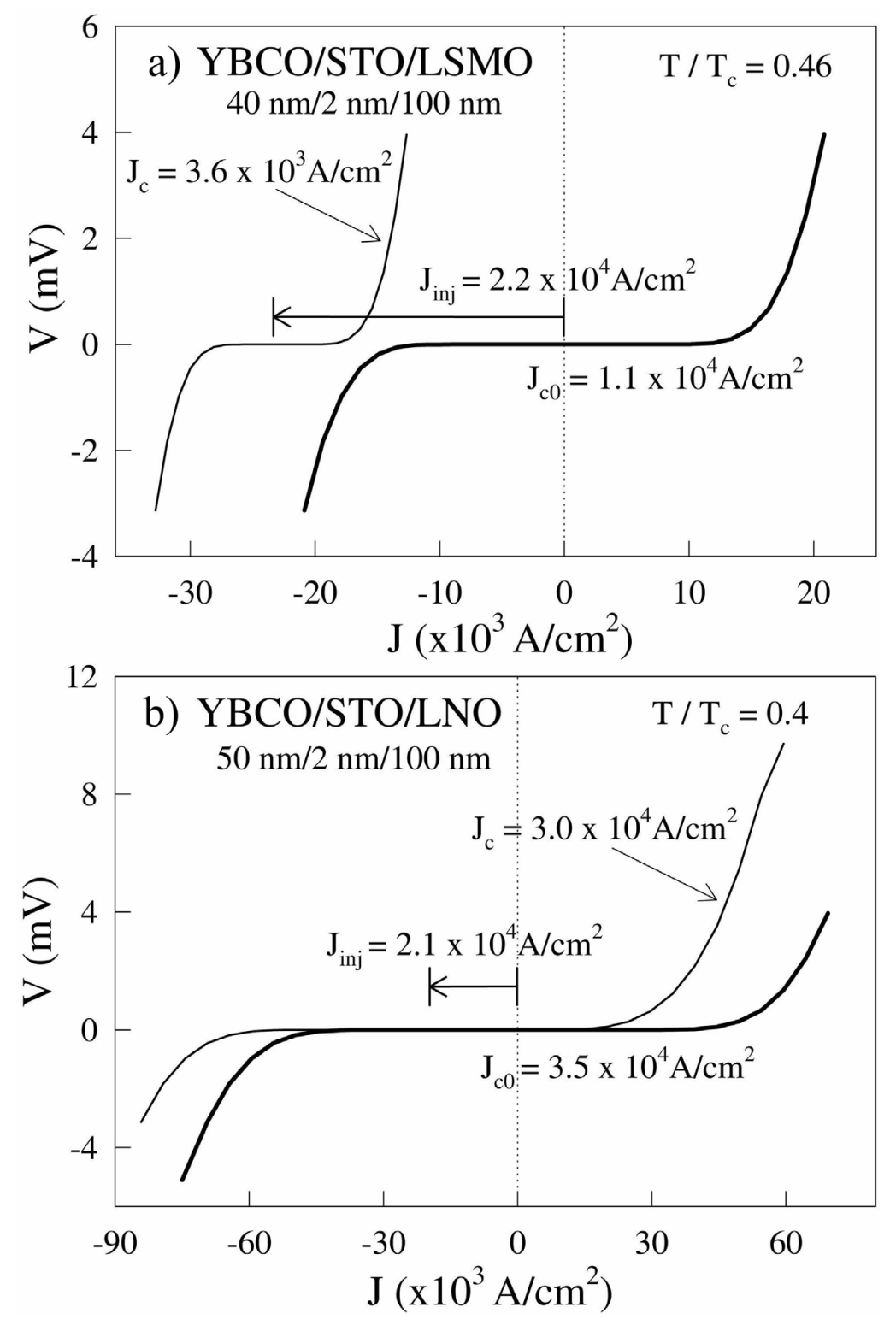

Figure 5.6: a) Representative current-voltage (I-V) characteristics of an F-I-S sample at $\left(T / T_{c}\right)=0.46$, showing a significant left shift of the I-V curve and a substantial suppression of $J_{c}$ upon injection of currents from a ferromagnetic layer. b) Representative I-V characteristics of an N-I-S sample at $\left(T / T_{c}\right)=0.4$, showing a much smaller left shift of the I-V curve and a weaker suppression of $J_{c}$ than those in the F-I-S upon injection of comparable currents from a non-magnetic layer [116]. 

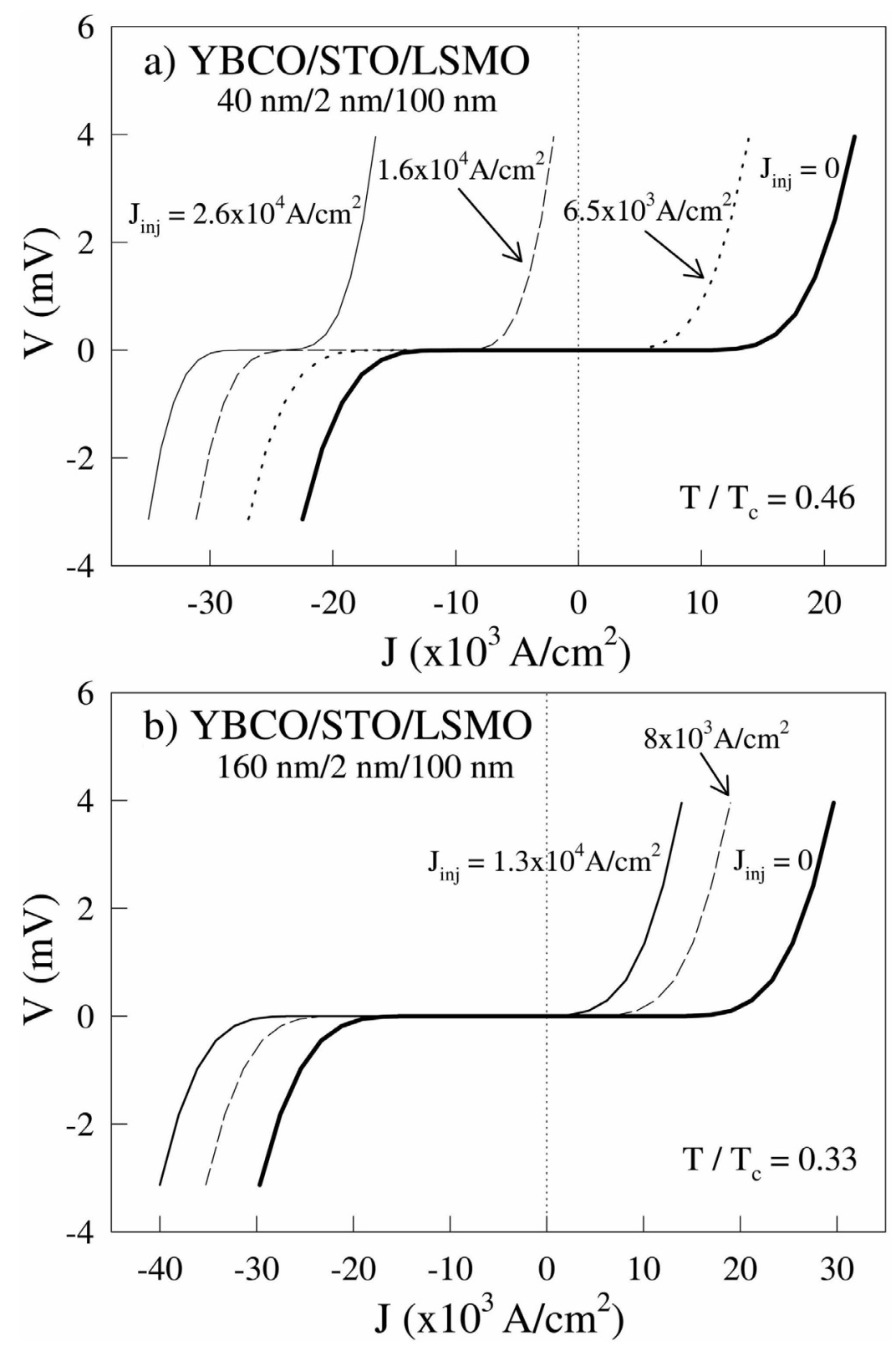

Figure 5.7: Representative I-V characteristics of F-I-S samples with YBCO thickness of a) $40 \mathrm{~nm}$ at $\left(T / T_{c}\right)=0.46$ and b) $160 \mathrm{~nm}$ at $\left(T / T_{c}\right)=0.33$ for a range of injection currents. This illustrates the more significant $J_{c}$ suppression observed in the thin YBCO heterostructure [116]. 


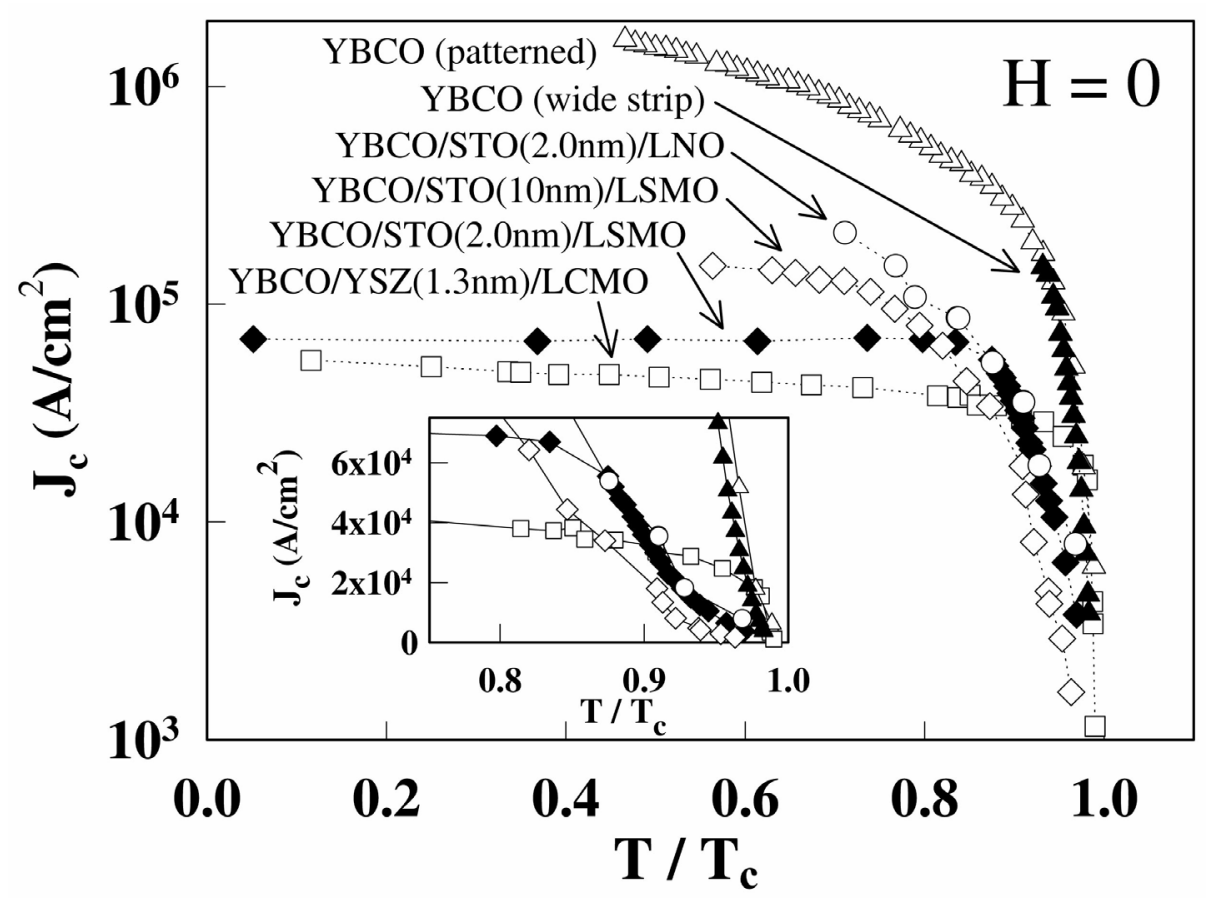

Figure 5.8: Comparison of the zero-field critical current density $\left(J_{c}\right)$ of various $100 \mathrm{~nm}$-thick YBCO and perovskite heterostructures as a function of the reduced temperature $\left(T / T_{c}\right)$. Details of $J_{c}(T)$ near $\left(T / T_{c}\right)=1$ are shown in the inset [53]. 

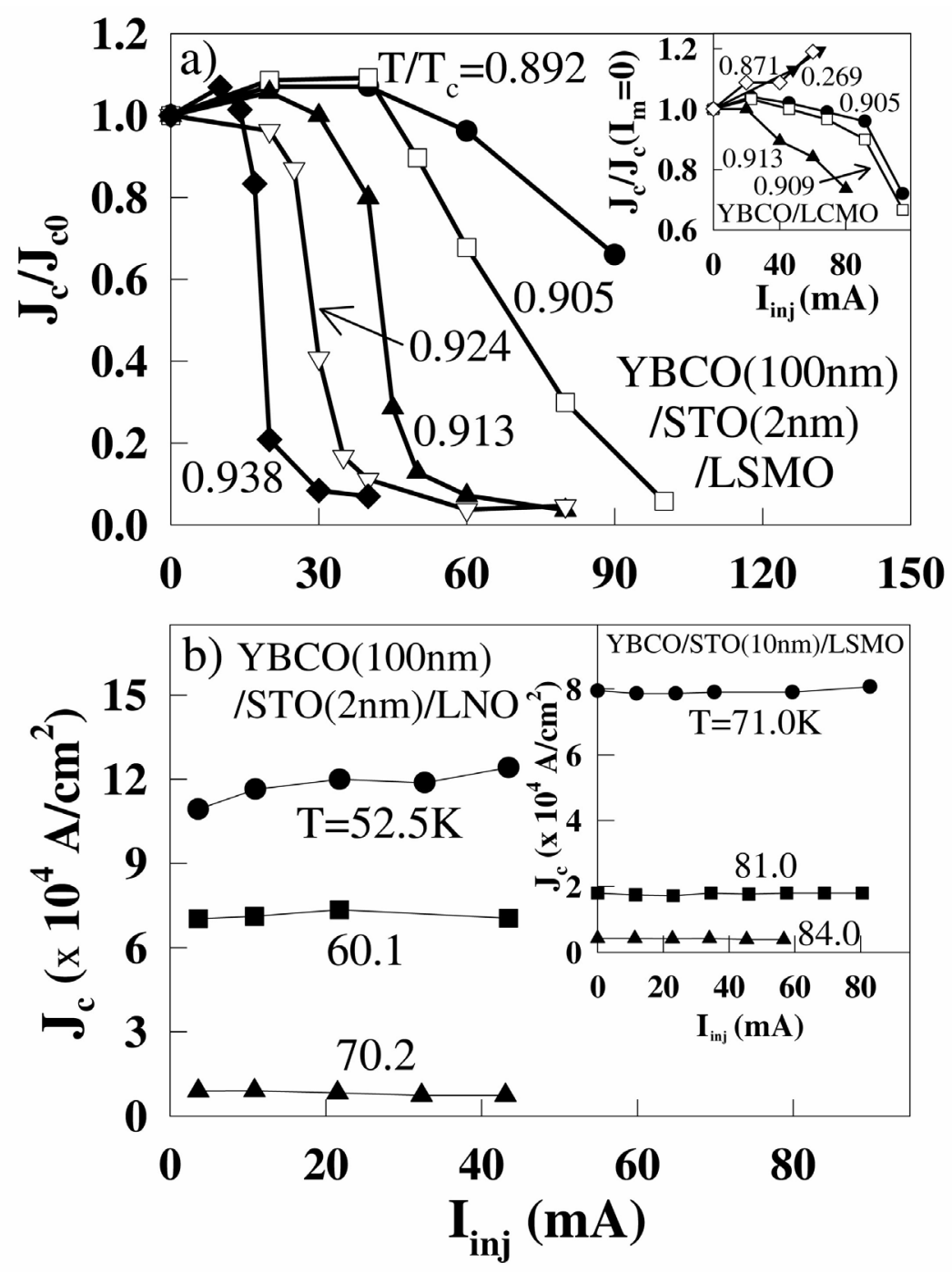

Figure 5.9: a) The $\left(J_{c} / J_{c 0}\right)$ vs. $I_{i n j}$ isotherms of YBCO/STO/LSMO $(100 / 2 / 100 \mathrm{~nm})$. The inset shows the corresponding results for YBCO/YSZ/LCMO (100/1.3/100 nm). b) The $J_{c}$ vs. $I_{i n j}$ isotherms of $\mathrm{YBCO} / \mathrm{STO} / \mathrm{LNO}(100 / 2 / 100 \mathrm{~nm})$ and YBCO/STO/LSMO $(100 / 10 / 100 \mathrm{~nm})$ are shown in the main panel and the inset, respectively [53]. 


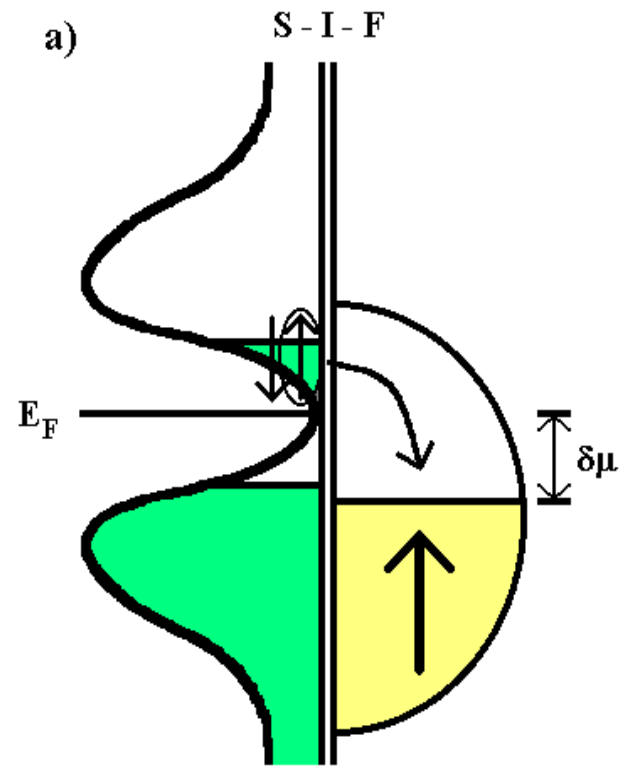

Self Injection

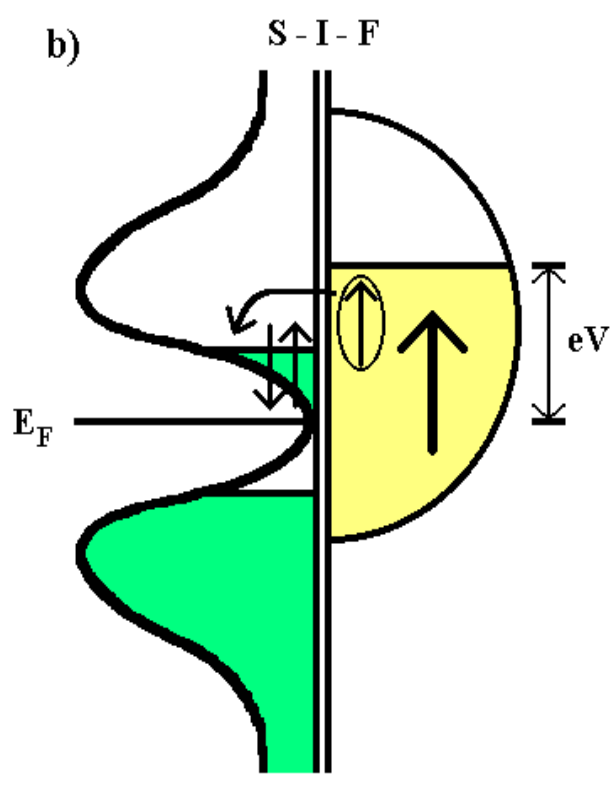

External Injection

Figure 5.10: a) Schematic illustration of the "self-injection" of spin-polarized quasiparticles near the F/S interface. b) Schematics of external injection of spin-polarized quasiparticles from $\mathrm{F}$ to $\mathrm{S}$. Here $E_{F}$ denotes the Fermi energy of the superconductor and $V$ is the external bias voltage across the $\mathrm{F} / \mathrm{S}$ interface. 

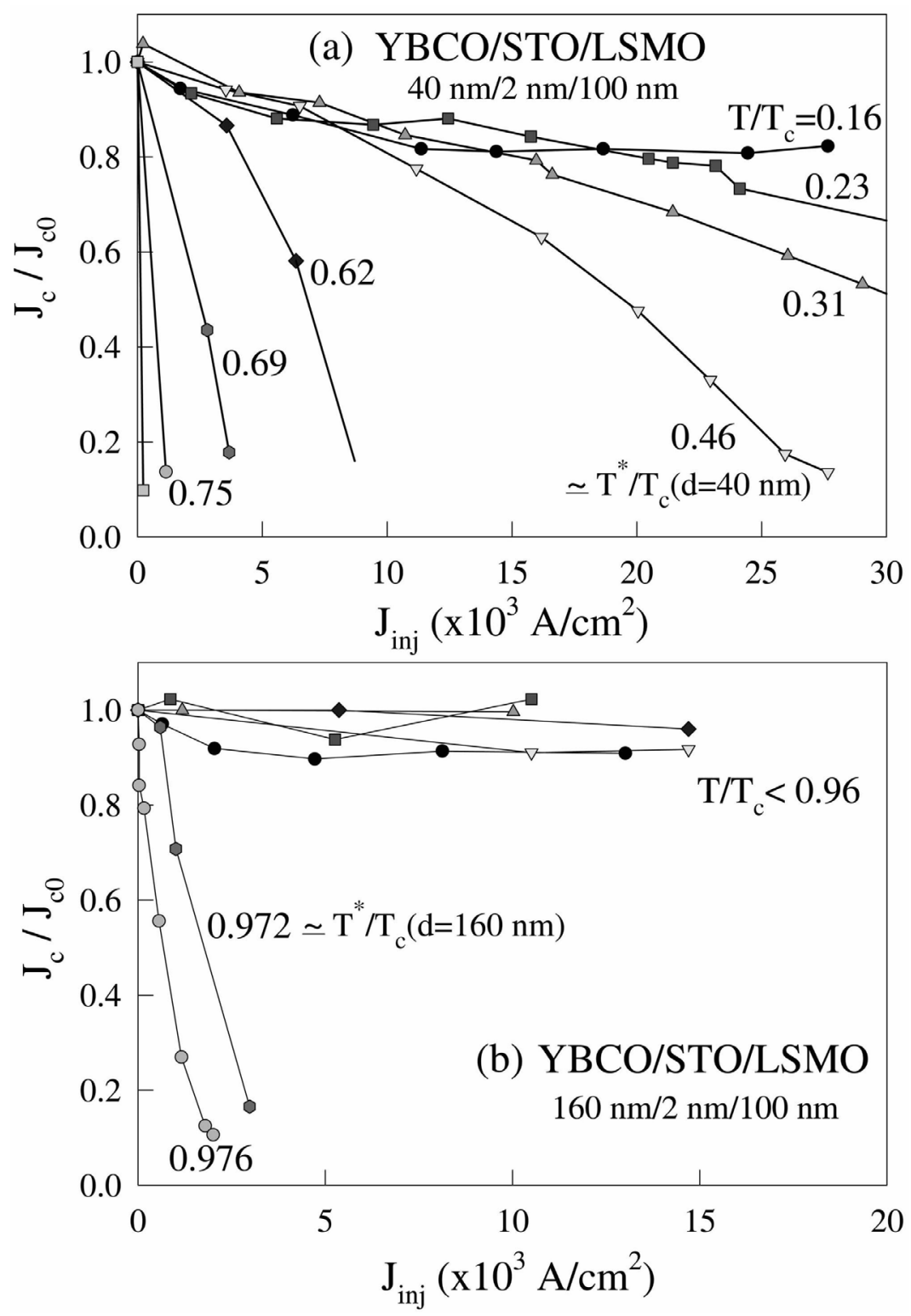

Figure 5.11: $J_{c^{-v s}}$-- $J_{i n j}$ isotherms of: a) an F-I-S sample with YBCO thickness $d=40 \mathrm{~nm}$; b) an F-I-S sample with YBCO thickness $d=160 \mathrm{~nm}[116]$. 

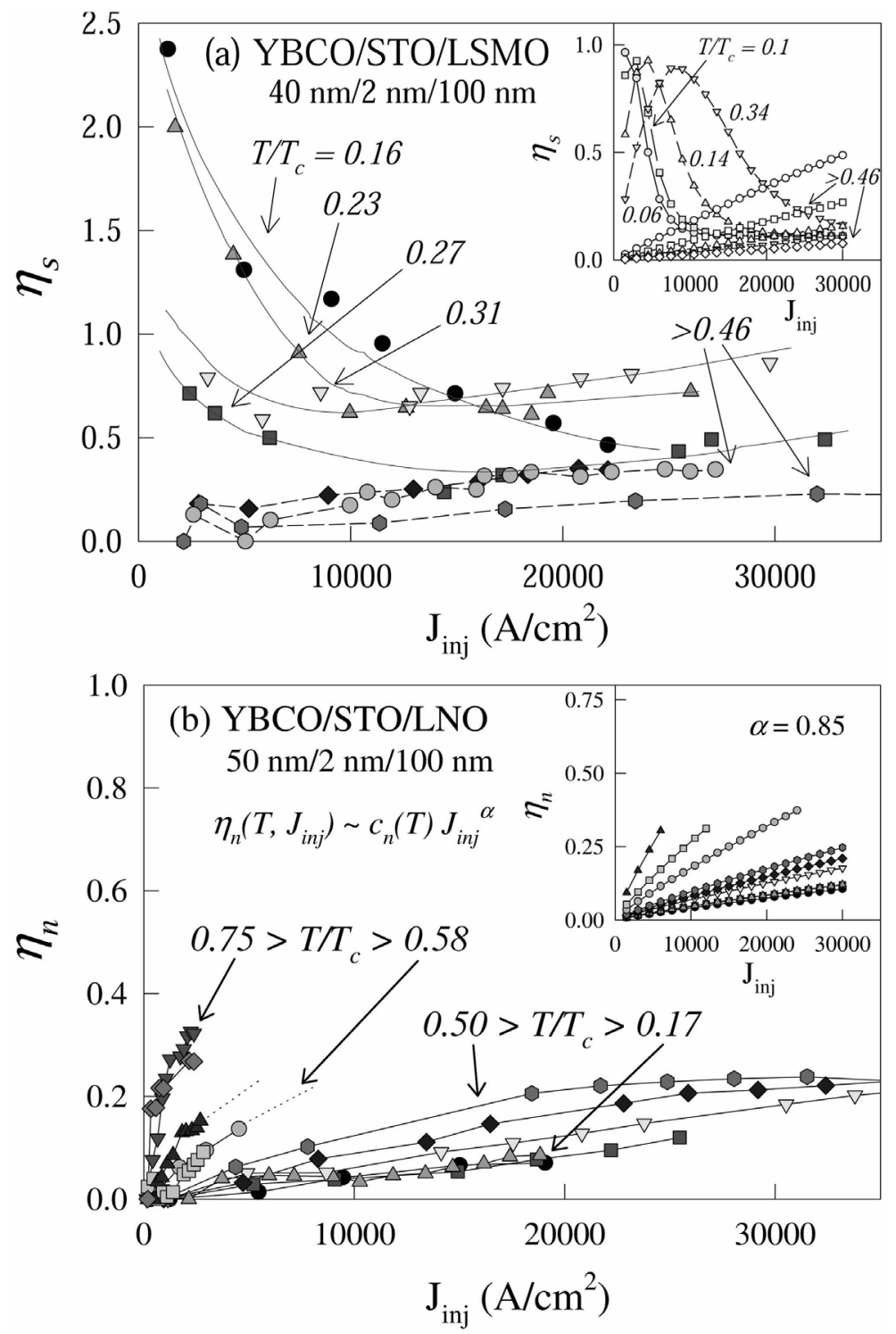

Figure 5.12: a) Efficiency $\eta_{s}$-vs.- $J_{i n j}$ isotherms of an F-I-S sample with YBCO thickness $d=40 \mathrm{~nm}$. b) Efficiency $\eta_{s}$-vs.- $J_{i n j}$ isotherms of an N-I-S sample with YBCO thickness $d=50 \mathrm{~nm}$. Insets: Simulated results discussed in Chapter 6 [116]. 


\section{Chapter 6}

\section{Analyses of Spin-Injection Results}

\subsection{Spin-relaxation Length Derivation}

To estimate the $c$-axis spin relaxation length $\delta_{s}^{c}(T)$ in YBCO, we empirically related the YBCO thickness $d$ of each F-I-S heterostructure to a characteristic reduced temperature $\left[T^{*}(d) / T_{c}\right]$ at which $\left(J_{c} / J_{c 0}\right) \leq 0.1$ is satisfied under a constant $J_{i n j}$. This assignment was based on the assumption that the observation of strong suppression in $J_{c}$ corresponded to the condition $\delta_{s}^{c} \rightarrow d$ for $\left(T / T_{c}\right) \rightarrow\left[T^{*}(d) / T_{c}\right]$, provided that the lateral dimensions of all samples were kept identical. Similar criterion could be applied to the N-I-S samples to define the $c$-axis charge relaxation length $\delta_{Q}^{c}$. The correlation of $\left(T^{*} / T_{c}\right)$ with the corresponding thickness $(d)$ of the F-I-S heterostructure is shown in Fig. 6.1, suggesting a characteristic length $\delta_{s}^{c}\left(\sim d\right.$ for $\left.\left(T / T_{c}\right) \rightarrow\left(T^{*} / T_{c}\right)\right)$ increased rapidly near $T_{c}$. The diverging characteristic length was attributed to a vanishing superconducting gap near $T_{c}$, [45] and was only detectable in the F-I-S heterostructures. We further remark that the diverging behavior in F-I-S samples was unlikely the result of any extrinsic effect such as systematically varying 


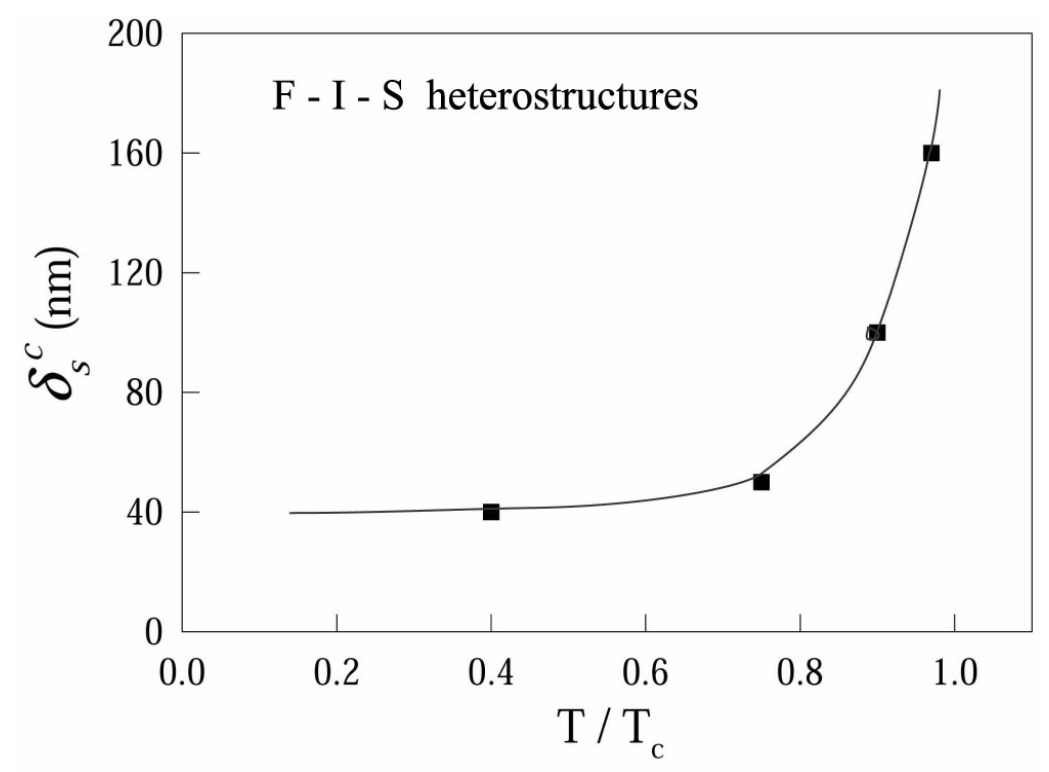

Figure 6.1: Characteristic $c$-axis spin relaxation length $\delta_{s}^{c}$ as a function of reduced temperature $\left(T / T_{c}\right)$ for the F-I-S heterostructures [116].

quality of YBCO with its thickness for the following reasons. All F-I-S samples had comparable $T_{c}$ while their $J_{c 0}$ values at $4.2 \mathrm{~K}$ were not monotonic with increasing film thickness, with minimum $J_{c 0} \sim 1.5 \times 10^{4} \mathrm{~A} / \mathrm{cm}^{2}$ associated with the sample of maximum thickness $d=160 \mathrm{~nm}$. Furthermore, in contrast to the observation in F-I-S samples, no obvious crossover temperature $T^{*}$ could be found in N-I-S samples for rapid decrease of $J_{c}$ with $J_{i n j}$. Thus, the occurrence of strong injection-induced superconductivity suppression (with $\left.J_{c} \lesssim 0.1 J_{c 0}\right)$ at larger values of $\left(T^{*} / T_{c}\right)$ for thicker F-I-S samples could not be ascribed to the result of better superconductivity in thicker YBCO.

The contrast in the temperature dependence of $\delta_{s}^{c}$ and of $\delta_{Q}^{c}$ for the F-I-S and NI-S samples could be attributed to the significantly longer lifetime of spin-polarized quasiparticles relative to that of simple quasiparticles $[48,46]$, so that the injection of simple quasiparticles did not result in complete suppression of $J_{c}$ in N-I-S samples for all temper- 
atures of our studies. In other words, the condition $J_{c}\left(T, J_{i n j}\right)<\sim 0.1 J_{c 0}(T)$ could not be realized in the N-I-S samples within our experimental resolution $[53,54]$, so that the charge relaxation length $\delta_{Q}^{c}$ appeared to be always smaller than $d$ for all temperatures of our study.

\subsection{Understanding Efficiency}

The seemingly surprising contrast between the F-I-S and N-I-S samples may be understood in the context of different quasiparticle relaxation mechanisms and nonequilibrium quasiparticle distributions. Generally speaking, an adequate description for nonequilibrium superconductivity must involve consideration of the quasiparticle energy $E_{\mathbf{k}}$ and the quasiparticle distribution function $f_{\mathbf{k}}$ in the superconductor, where $\mathbf{k}$ denotes the quasiparticle momentum. In principle, an explicit expression for $E_{\mathbf{k}}$ can be obtained by solving the Bogoliubov-de Gennes equations [120, 45], provided that the exact Hamiltonian $\mathcal{H}$ for the superconductor is known. In thermodynamic equilibrium, the quasiparticle energy associated with the unperturbed Hamiltonian $\mathcal{H}_{0}$ is $E_{\mathbf{k} 0}=\left(\Delta_{\mathbf{k}}^{2}+\xi_{\mathbf{k}}^{2}\right)^{1 / 2}$, where $\xi_{\mathbf{k}}\left(\equiv \varepsilon_{\mathbf{k}}-\varepsilon_{F}\right)$ is the single particle energy $\varepsilon_{\mathbf{k}}$ relative to the Fermi level $\varepsilon_{F}$, and $\Delta_{\mathbf{k}}$ is the pairing potential $[120,45]$. For an $s$-wave superconductor $\Delta_{\mathbf{k}}$ is a constant, whereas for a pure $d_{x^{2}-y^{2}}$-pairing superconductor, $\Delta_{\mathbf{k}} \approx \Delta_{d} \cos 2 \theta_{k}$, and $\theta_{k}$ is an angle measured from one of the antinodes of the order parameter in momentum space $[8,9]$. The injection of external quasiparticles is expected to interact with the superconductor through an interaction Hamiltonian $\mathcal{H}_{I}$ and to modify the quasiparticle energy and the distribution of quasiparticle states through the total Hamiltonian $\mathcal{H}=\mathcal{H}_{0}+\mathcal{H}_{I}$, provided that the perturbative approximation is valid. In the absence of available theory for nonequilibrium quasiparticle distributions in a strongly 
correlated $d$-wave superconductor, we consider in the following analyses of our data based on conventional theory of nonequilibrium superconductivity, and discuss the implication of results thus derived from F-I-S and N-I-S samples.

Consider a simple case where a uniform supercurrent with a velocity $\vec{v}_{s}=\vec{J}_{s} /\left(n_{s} e\right)$ exists in the superconductor. The finite momentum associated with the supercurrent $J_{s}$ is found to change the quasiparticle energy $E_{\mathbf{k} n}$ and the distribution function $f_{\mathbf{k} n}$ as follows $[45]:$

$$
\begin{aligned}
E_{\mathbf{k} n} & =E_{\mathbf{k} 0}+\hbar \mathbf{k}_{F} \cdot \mathbf{v}_{s} / m^{*} \equiv E_{\mathbf{k} 0}+\delta E_{J}, \\
f_{\mathbf{k} n} & =\left\{1+\exp \left[E_{\mathbf{k} n} /\left(k_{B} T\right)\right]\right\}^{-1},
\end{aligned}
$$

where $\mathbf{k}_{F}$ is the quasiparticle momentum at the Fermi level. Using the $d_{x^{2}-y^{2}}$-wave pairing potential in optimally doped $\mathrm{YBCO}$ with $\Delta_{d} \approx 30 \mathrm{meV},[11,62,12]$ and the single particle energies $\varepsilon_{\mathbf{k}}$ derived from the tight-binding band structure calculations [121] with parameters tabulated in Table 6.1 , we find that $E_{\mathbf{k} 0} \gg\left|\delta E_{J}\right|$ is satisfied for typical supercurrents $\left(J_{c}=10^{4} \sim 10^{5} \mathrm{~A} / \mathrm{cm}^{2}\right.$ for $\left.T \ll T_{c}\right)$ sustainable in the YBCO superconducting layers. Thus, we expect $f_{\mathbf{k} n} \sim 0$ if $T \ll T_{c}$.

In the event that a quasiparticle current $J_{i n j}$ is externally injected into a superconductor that already carries a supercurrent $J_{s}$, the situation becomes more complex because the externally injected quasiparticles must redistribute themselves among available states that obey the Pauli exclusion principle for fermions, and the redistribution must involve inelastic and elastic scattering processes. Therefore the injected quasiparticle momenta relative to the supercurrent direction and the lattice momenta are not well defined due to the involvement of inelastic scattering processes [45], and it is not uncommon that a 
current-carrying superconductor with an initial supercurrent $J_{s}$ can remain superconducting under an external injection current $J_{i n j}$ such that the sum of $J_{i n j}$ and $J_{s}$ exceeds the critical current $J_{c 0}$ of the superconductor [45], as exemplified in Fig. 5.6(a). Nonetheless, we find that the simple sum of the maximum injection current density and the supercurrent density still yields $\max \left[\hbar \vec{k}_{F} \cdot\left(\vec{J}_{s}+\vec{J}_{i n j}\right) /\left(n_{s} e\right)\right] \ll E_{k 0}$ at low temperatures. Thus, the excess momentum due to external quasiparticle injection is insufficient to yield significant redistribution of quasiparticles. For N-I-S samples with relatively thin YBCO, the small yet monotonically increasing $\Delta J_{c}$ with increasing $J_{i n j}$ and increasing $T$ (see Fig. 5.12(b)) should be attributed primarily to the increasing normal fluid density and the suppression of the superconducting phase stiffness.

More specifically, the efficiency associated with simple quasiparticle injection in the N-I-S samples may be given by the following phenomenological expression:

$$
\eta_{n} \equiv \frac{1}{N_{0}} \sum_{k}\left(1-2 f_{k n}\right) c_{n}(T) g\left(J_{i n j}\right) \approx c_{n}(T) g\left(J_{i n j}\right)
$$

where $N_{0}$ denotes the total number of quasiparticle states, $c_{n}(T)$ is associated with the temperature-dependent fraction of the normal fluid and is a monotonic function of $T, g\left(J_{i n j}\right)$ reflects the weakening of the superconducting phase stiffness under increasing $J_{i n j}$ and is a monotonic function of $J_{i n j}$, and the quantity $\left(1-2 f_{k n}\right)$ ensures no double occupancy of the quasiparticle states. However, we have found $f_{k n} \approx 0$ for the entire range of $T$ and $J_{i n j}$ of our interest, so that $N_{0}^{-1} \sum_{k}\left(1-2 f_{k n}\right) \approx 1$. Empirically, we find that $c_{n}(T) \sim$ $\left\{1-\left[1-\left(T / T_{c}\right)\right]^{\nu}\right\}$ where $0.5<\nu<0.9$ and $g\left(J_{i n j}\right) \sim\left(J_{i n j}\right)^{0.85}$. The simulated example of $\eta_{n}$-vs.- $J_{i n j}$ isotherms using Eq. (6.2) and the value $\nu \approx 0.6$ are shown in the inset of Fig. 5.12(b) for comparison with the experimental data. 


\begin{tabular}{|c|c|}
\hline Parameter & Value \\
\hline$\Delta_{d}$ & $30 \mathrm{meV}$ \\
\hline$\varepsilon_{F}$ & $0.51 \mathrm{eV}$ \\
\hline$t$ & $0.18 \mathrm{eV}$ \\
\hline$t^{\prime}$ & $50 \mathrm{meV}$ \\
\hline$\gamma$ & 1.02 \\
\hline$v_{F}$ & $2 \times 10^{5} \mathrm{~m} / \mathrm{s}^{2}$ \\
\hline$l_{t r}{ }^{c}$ & $\sim 1 \mathrm{~nm}$ \\
\hline
\end{tabular}

Table 6.1: The values and references of various physical parameters used in computing $\eta_{s}$ and $\eta_{n}$ are tabulated. Here $t$ and $t^{\prime}$ are the nearest and next-nearest neighbor interaction integrals in the two-dimensional tight-binding model for the normal state energy bandstructure of the $\mathrm{CuO}_{2}$-plane.

In contrast, significantly different response of the cuprate superconductors to the injection of spin-polarized quasiparticles is expected because of their relatively longer lifetime and their strong effects on suppressing the $\mathrm{Cu}^{2+}-\mathrm{Cu}^{2+}$ antiferromagnetic correlation. Although we do not know the exact interaction Hamiltonian for the spin-polarized quasiparticles in the cuprate superconductors and therefore cannot obtain the quasiparticle energy $E_{\mathbf{k} s}$, it seems informative to estimate the approximate quasiparticle energy by applying conventional theory for nonequilibrium quasiparticle distribution under charged particle injection to the cuprate superconductors. That is, the perturbative approximation as manifested by an effective chemical potential shift $\mu^{*}$ in the single particle energy $\xi_{\mathbf{k}}$ is assumed to be valid $[65,68,45]$. Noting that $J_{c}$ is obtained by identifying the onset of dissipation where the maximum magnitude of the $d_{x^{2}-y^{2}}$-wave superconducting gap has been driven to a small value, we consider the situation similar to that for a gapless superconductor [120]. 
Thus, the quasiparticle energy under spin injection may be approximated by

$$
\begin{aligned}
E_{\mathbf{k} s} & =\left[\left(\xi_{\mathbf{k}}-\mu^{*}\right)^{2}+\Delta_{\mathbf{k}}^{2}\right]^{1 / 2} \\
& \approx\left|\xi_{\mathbf{k}}-\mu^{*}\right|\left[1+\frac{\Delta_{k}^{2}}{2\left(\xi_{\mathbf{k}}-\mu^{*}\right)^{2}}\right]
\end{aligned}
$$

where $\Delta_{k} \ll\left|\xi_{\mathbf{k}}-\mu^{*}\right|$, and the corresponding quasiparticle distribution function becomes

$$
f_{\mathbf{k} s}\left(T, J_{i n j}\right)=1 /\left\{1+\exp \left[E_{\mathbf{k} s} /\left(k_{B} T\right)\right]\right\}
$$

The chemical potential shift per quasiparticle $\mu^{*}$ due to spin injection must satisfy the conditions $\mu^{*} \rightarrow 0$ for $J_{i n j} \rightarrow 0$ and $\mu^{*} \rightarrow$ constant $\equiv \mu_{00}^{*}$ for large $J_{i n j}$ and low temperatures, where $\mu_{00}^{*}$ is a constant. Therefore a reasonable approximation for $\mu^{*}$ can be given by $\mu^{*}=\mu_{00}^{*} \tanh \left(c_{1} J_{i n j} / k_{B} T\right)$, and the physical significance of the functional form $\tanh \left(c_{1} J_{i n j} / k_{B} T\right)$ is consistent with the average spin polarization $(P)$ per quasiparticle in the superconductor. Here

$$
c_{1} J_{i n j}=\mu_{B}\left[\mu_{0}\left(\left\langle m_{s}\right\rangle P / \Omega\right)\right]
$$

is associated with the effective field energy, $\left\langle m_{s}\right\rangle$ denotes the excess magnetic moments in the superconductor due to spin injection, $\mu_{B}$ is the Bohr magneton, $\mu_{0}$ is the vacuum permeability, and $\Omega=\mathcal{A} d$ is the superconducting volume. In the absence of a known interaction Hamiltonian, $\mu_{00}^{*}$ and $c_{1}$ are positive quantities to be determined empirically. On the other hand, simple dimensional analysis yields

$$
\left\langle m_{s}\right\rangle=\left(\mu_{B} / e\right) I_{i n j} \tau_{s}=\left(\mu_{B} / e\right)\left(J_{i n j} \mathcal{A} \tau_{s}\right)
$$

where $\tau_{s}$ is the in-plane spin dephasing time.

Anticipating suppression in the critical current density due to the presence of excess magnetic moments, $\Delta J_{c}$ is linked to the effective magnetization $\left(\left\langle m_{s}\right\rangle P / \Omega\right)$ adjusted 
by the available quasiparticle states. That is,

$$
\begin{aligned}
\Delta J_{c} & \equiv\left(J_{c 0}-J_{c}\right) \propto \sum_{k}\left(1-2 f_{k s}\right)\left(\left\langle m_{s}\right\rangle P / \Omega\right), \\
& \propto \sum_{k}\left(1-2 f_{k s}\right) J_{i n j} \tanh \left(c_{1} J_{i n j} / k_{B} T\right),
\end{aligned}
$$

where the effective magnetization $\left(\left\langle m_{s}\right\rangle P / \Omega\right)$ takes the form of the Brillouin function for a spin-1/2 system, and the quantity $\left(1-2 f_{k s}\right)$ ensures no double occupancy in the quasiparticle states. As a result, $\eta_{s}$ becomes

$$
\eta_{s} \equiv \Delta J_{c} / J_{i n j} \sim \frac{1}{N_{0}} \sum_{k}\left(1-2 f_{k s}\right) \tanh \left(c_{1} J_{i n j} / k_{B} T\right)
$$

Strictly speaking, the quantity $\left(1-2 f_{k s}\right)$ in Eq. (6.8) should have been written as $\left(1-f_{\uparrow}-f_{\downarrow}\right)$, where the $\uparrow$ spin polarization corresponds to that parallel to the effective magnetic field induced by the excess magnetic moments. However, the effective field can be shown to be very small, as discussed in Sec. 6.3. Consequently, we find $\left(1-f_{\uparrow}-f_{\downarrow}\right) \approx\left(1-2 f_{k s}\right)$.

Following the analysis outlined above and inserting the relevant experimental parameters as tabulated in Table 6.1 into Eq. (6.8), results similar to the experimental findings are obtained for the F-I-S sample with the thinnest YBCO in which the effects of spin injection were fully realized over a wide temperature range, as shown in the inset of Fig. 5.12(a). However, a quantitative agreement with the experimental data for the thinnest F-I-S sample could only be achieved by invoking a large chemical potential shift $\mu_{00}^{*}$ associated with $f_{k s}$, so that $\mu^{*}$ varied from $\sim 700 \mathrm{meV}$ at $T \ll T_{c}$ to $\sim 45 \mathrm{meV}$ at $T \rightarrow T_{c}$. In Fig. 6.2, the functional form of $c_{1}(T)$ used in the data fitting is shown, which determines empirically the temperature dependence of $\mu^{*}$. These values are unusually large, comparable to the bandstructure parameters. Interestingly, the empirical value $\mu_{00}^{*}$ for $T \ll T_{c}$ is comparable 
to $4 J_{e x}$ in the YBCO system, where $J_{e x}$ is the nearest neighbor antiferromagnetic coupling constant, and the factor of 4 corresponds to the number of nearest neighbors in the square lattice of the $\mathrm{CuO}_{2}$ plane. While these large values of $\mu_{00}^{*}(T)$ are likely unphysical and should not be taken literally because of the questionable validity of applying conventional theory to cuprate superconductivity, the following conclusions may be drawn from our analyses. First, the large magnitude of $\mu_{00}^{*}$ found only in F-I-S implies strong effects of spin injection on cuprate superconductors, as opposed to the negligible change in the chemical potential of N-I-S samples under current injection. Second, the large $\mu_{00}^{*}$ values in F-I-S suggest the breakdown of conventional perturbative approximation to the interaction Hamiltonian of nonequilibrium superconductors. That is, a valid perturbative approximation would have yielded a small chemical potential shift relative to the relevant bandstructure parameters in the single-particle energy $\xi_{\mathbf{k}}$. That the chemical potential shift derived from perturbative approximation turned out to be comparable to the bandstructure parameters is suggestive of strong interaction effects associated with spin-polarized quasiparticles in cuprate superconductors and thus the failure of perturbative approximation.

Despite the uncertainty in the magnitude of the chemical potential, the temperature dependence of $\mu^{*}$ is directly related to that of the effective magnetization, and therefore can provide information for the spin relaxation process. In particular, for the F-I-S sample with the thinnest YBCO layer $(d=40 \mathrm{~nm})$, the spin injection effects were already realized at low temperatures, suggesting that the $c$-axis spin relaxation length was either comparable to or exceeding the sample thickness over most temperatures of our investigation. Hence, the temperature evolution of the spin-dependent information deduced from those data may 


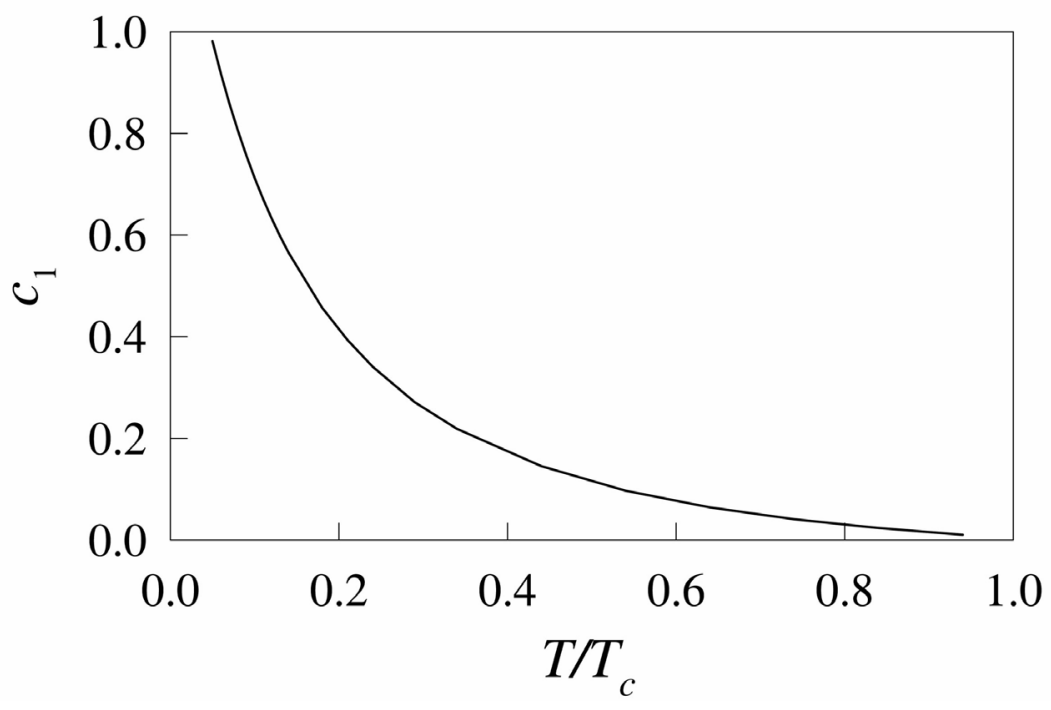

Figure 6.2: Functional form of $c_{1}(T)$ used in the simulation of $\eta_{s}$.

be considered to be primarily associated with that of the in-plane spin relaxation. In contrast, measurements on F-I-S samples with thicker YBCO contained convoluted information for both the $c$-axis and in-plane spin relaxation processes over most temperatures except near $T_{c}$, and therefore could not be used to infer direct information associated with the in-plane spin relaxation. Thus, the empirically determined coefficient $c_{1}(T)$ in Eq. (6.8) for the F-I-S sample with $d=40 \mathrm{~nm}$ could be approximately related to an effective in-plane spin relaxation time $\tau_{s}(T)$ by $c_{1}(T) \approx \mu_{0} \mu_{B}^{2} \tau_{s} /(e d)$ according to Eqs. (6.5) and (6.6), and we find that $\tau_{s}(T)$ ranges from $\sim 10^{-4} \mathrm{~s}$ at $T \ll T_{c}$ to $\sim 10^{-6} \mathrm{~s}$ at $T \rightarrow T_{c}^{-}$. Such a long characteristic time scale is comparable to the spin-spin relaxation time obtained from the nuclear quadruple resonance (NQR) experiments [122], and is approximately one-totwo orders of magnitude longer than the in-plane simple quasiparticle recombination time determined from measurements of photo-induced activation of microwave absorption [64]. 
The above phenomenological analyses suggest that the injection of spin-polarized quasiparticles in YBCO appeared to exert strong influence on the microscopic quasiparticle energy and density of states (DOS), probably through exchange interaction with the short-range $\mathrm{Cu}^{2+}-\mathrm{Cu}^{2+}$ antiferromagnetic correlation. Furthermore, the slower relaxation of spin-polarized quasiparticles relative to the already long recombination time of simple quasiparticles [64] appeared reasonable because of the further reduced probability of quasiparticle recombination before excess spin polarization can be relaxed. It is also interesting to compare the transport data presented here with our scanning tunneling spectroscopic studies of YBCO in the F-I-S and N-I-S heterostructures that revealed significantly modified quasiparticle DOS at $4.2 \mathrm{~K}$ under spin injection and no discernible changes under simple quasiparticle injection [55]. The spectroscopic studies are not only supportive for our finding of significantly longer relaxation time of spin-polarized quasiparticles relative to that of simple quasiparticles, but also suggestive of direct influence of spin injection on the microscopic states of the cuprates.

Next, we consider the appearance of a diverging $c$-axis spin relaxation length near $T_{c}$. In conventional superconductors, it is known that the characteristic quasiparticle relaxation time $\tau_{Q}$ can diverge near $T_{c}$ due to the vanishing superconducting gap $\Delta(T)$ through the following relation $[48,45]$ :

$$
\tau_{Q}(T) \approx \frac{4 \tau_{E} k_{B} T_{c}}{\pi \Delta(T)}
$$

where $\tau_{E}$ is the inelastic electron-phonon scattering time, and $\Delta(T)=\Delta_{0}\left[1-\left(T / T_{c}\right)\right]^{\nu}$, with $\Delta_{0}$ being the zero-temperature superconducting gap and $\nu$ the order-parameter critical exponent. This diverging behavior due to weakening superconductivity gives rise to 
stronger effects of quasiparticle injection with increasing temperature near $T_{c}$. The temperature interval for revealing such divergence depends on the critical fluctuation regime and also on the temperature dependence of $\tau_{E}$, and is generally very narrow in conventional superconductors, because $\tau_{Q}$ decreases rapidly with decreasing temperature and competes with other characteristic times (such as the quasiparticle recombination time) at low temperatures. On the other hand, the critical fluctuation regime of cuprate superconductors is known to be several orders of magnitude larger than that of the conventional superconductors [123]. In the case of $\mathrm{YBCO}$, the critical regime associated with the zero-field transition temperature $T_{c}$ is estimated at approximately $1 \% \sim 10 \%$ of $T_{c}$. Hence, it is in principle more promising to observe this diverging quasiparticle relaxation length in the cuprates near $T_{c}$.

In the previous section, we have attributed the rapidly increasing characteristic length near $T_{c}$ in the F-I-S samples (see Fig. 6.1) to the $c$-axis spin relaxation length $\delta_{s}^{c}$. Whereas the transport of spin-polarized quasiparticles actually took place along both inplane and $c$-axis, this attribution is still reasonable because the $c$-axis dimensions of all F-I-S samples were several orders of magnitude smaller than the lateral dimensions and therefore were most sensitive to the crossover of a $c$-axis relaxation length to the sample thickness. Consequently, the temperature dependence of $\delta_{s}^{c}$ could be related to a $c$-axis spin relaxation time $\tau_{s}^{c}$, at least semi-quantitatively. If the spin transport along $c$-axis is diffusive and if no spin-charge separation exists, we have $\delta_{s}^{c}=\sqrt{D_{s}^{c} \tau_{s}^{c}}$, where $D_{s}^{c}=\left(v_{F} \ell_{t r}^{c}\right) /\left(3 \lambda_{s o}\right)$ is the $c$-axis spin diffusion coefficient and $\ell_{t r}^{c}$ is the transport mean-free path along the $c$-axis, $\lambda_{s o}(\sim 0.1)$ is the dimensionless spin-orbit coupling constant [44], and $\tau_{s}^{c}$ is associated with the inelastic 
spin-orbit scattering time $\tau_{\text {so }}$ via a relation similar to that in Eq. (6.9):

$$
\tau_{s}^{c}(T) \approx \frac{4 \tau_{s o} k_{B} T_{c}}{\pi\left\langle\left|\Delta_{k}(T)\right|\right\rangle_{k}}
$$

Here $\left\langle\left|\Delta_{\mathbf{k}}(T)\right|\right\rangle_{\mathbf{k}}$ denotes the angular average of the $d$-wave gap, and the temperature dependence of $\left|\Delta_{\mathbf{k}}(T)\right|$ is approximated by $\left|\Delta_{\mathbf{k}}(T)\right| \sim\left[1-\left(T / T_{c}\right)\right]^{\nu}$. Assuming that $\tau_{s o}$ is a weak function of the temperature, we compare the $\delta_{s}^{c}$ value $(\sim 40 \mathrm{~nm})$ at $\left(T / T_{c}\right)=0.1$ with that $(\sim 160 \mathrm{~nm})$ at $\left(T / T_{c}\right)=0.9$ and use Eq. $(6.10)$ to obtain the order-parameter exponent $\nu \approx 0.65$, which is consistent with the exponent $\nu=2 / 3$ for the XY-model. Furthermore, using Eq. (6.10), the physical parameters $v_{F}$ and $\ell_{t r}^{c}$ listed in Table 6.1, and the empirical values of $\delta_{s}^{c}(T)$, we find $\tau_{s o} \approx 10^{-11} \sim 10^{-10} \mathrm{~s}$, which is consistent with the theoretical estimate for spin-orbit interaction [44]. We therefore suggest that $c$-axis spin relaxation mechanism may be dominated by the spin-orbit interaction, and the relaxation time $\tau_{s}^{c}$ is substantially shorter than that associated with the in-plane spin relaxation, implying anisotropic spin transport.

Concerning the $c$-axis simple quasiparticle transport, we remark that the overall effects of current injection in the N-I-S samples depend strongly on the transmission and energy relaxation of simple quasiparticles along the $c$-axis, and are therefore sensitive to the inter-planar inelastic scattering mechanism in addition to the in-plane quasiparticle recombination. Given that the $c$-axis dimensions of the N-I-S samples were much smaller than the lateral dimensions, the overall effects of simple quasiparticle injection should be primarily determined by the magnitude of the $c$-axis simple quasiparticle relaxation length $\delta_{n}^{c}$ relative to the sample thickness, even though the in-plane recombination time of excess simple quasiparticles can be relatively long due to the existence of nodes in the pairing 
potential [64]. Taking Eq. (6.9) and the typical electron-phonon scattering time in the cuprates, $\tau_{E} \sim 10^{-11} \mathrm{~s}$ for $\left(T / T_{c}\right) \ll 1$ and $\tau_{E} \sim 10^{-13} \mathrm{~s}$ for $\left(T / T_{c}\right) \rightarrow 1$, we obtained $\delta_{n}^{c}=\sqrt{D_{n}^{c} \tau_{Q}}$ that ranges from $\sim 20 \mathrm{~nm}$ at $\left(T / T_{c}\right) \sim 0.1$ to $<\sim 5 \mathrm{~nm}$ at $\left(T / T_{c}\right) \sim 0.9$, where $D_{n}^{c}=v_{F} \ell_{t r}^{c} / 3$ is the charge diffusion coefficient along $c$-axis. These estimates are consistent with the negligible effect of current injection in the N-I-S samples with a thick superconducting layer $(\sim 100 \mathrm{~nm})$, and the finite (although relatively small) suppression of $J_{c}$ in those N-I-S samples with a thinner superconducting layer $(\sim 50 \mathrm{~nm})$. Due to the rapid decrease in the electron-phonon scattering time $\tau_{E}$ with $T$ near $T_{c}$, a diverging $\delta_{n}^{c}(T)$ can only be expected if temperature becomes sufficiently close to $T_{c}$ so that the increasing value of $\langle\Delta(T)\rangle^{-1}$ with $T$ compensates for the decreasing $\tau_{E}(T)$. A simple estimate using Eq. (6.9) suggests that $0.999 T_{c}<T<T_{c}$ would be necessary to manifest the diverging simple quasiparticle relaxation length, which is beyond our experimental resolution for measurements of the corresponding $J_{c}$.

\subsection{Discussion}

The phenomenological analyses based on conventional theory of nonequilibrium superconductivity in the previous section suggest significant effects of spin-injection on cuprate superconductivity and anisotropic spin transport, with spin relaxation probably dominated by the spin-orbit interaction along $c$-axis and by the exchange interaction within the $\mathrm{CuO}_{2}$ plane. Under the premise of high-quality F-I-S heterostructures and interfaces, the significant influence of spin-polarized quasiparticles on the microscopic DOS is likely unique to the cuprate superconductors because of the strong correlation between the con- 
ducting holes and spin fluctuations $[19,5,6]$. Such drastic dynamic effects on cuprate superconductivity are reminiscent of the suppression of superconductivity and long-range effects induced by static non-magnetic impurities that substitute the $\mathrm{Cu}^{2+}$ ions and induce magnetic moments on the surrounding nearest-neighbor $\mathrm{Cu}^{2+}$ ions in the $\mathrm{CuO}_{2}$ planes $[31,32,33,34,35,36,37,38,39,40,41,10]$. The short-range antiferromagnetic correlation has been considered to play a significant role in the cuprate superconductivity, and the static non-magnetic impurities in the $p$-type cuprates are believed to have broken the antiferromagnetic correlation of $\mathrm{Cu}^{2+}$ ions [30], thus inducing localized magnetic moments and resulting in strong suppression of the collective spin excitation and the global pairing potential $[38,35,39,41,10]$. Similarly, we consider that the continuous injection of spin-polarized quasiparticles into the cuprate superconductors has effectively resulted in a quasi-static ferromagnetic perturbation to the antiferromagnetic correlation in the $\mathrm{CuO}_{2}$ planes, thereby yielding strong effects and slow relaxation in the quasiparticle spectra.

\subsection{Alternative Scenarios}

\subsubsection{Paramagnetic Effect}

Next, we comment on the possible relevance of paramagnetic effect $[120,124]$ to the observed suppression of cuprate superconductivity due to spin injection. We consider the spatially averaged effective magnetic induction $B_{\text {eff }}$ due to an injected spin-polarized current density $J_{i n j}$. Assuming the $c$-axis spin de-phasing time $\tau_{s}^{c}$ and taking the polarization $P=1$ for simplicity, we obtain an upper bound for $B_{\text {eff }}$ :

$$
B_{e f f} \leq \mu_{0}\left(\mu_{B} / e\right)\left(J_{i n j} \tau_{s}^{c} / d\right)
$$


where $\tau_{s}^{c}$ is related to $\tau_{s o}$ as given in Eq. (6.10). Thus, we obtained $B_{e f f} \sim 10^{-4}$ Tesla for $d=100 \mathrm{~nm}$ and $J_{i n j}=10^{5} \mathrm{~A} / \mathrm{cm}^{2}$. This effective field is clearly insignificant compared with any critical fields of the superconductor, thus cannot account for the strong suppression of superconductivity under the injection of spin-polarized quasiparticles.

\subsection{2 "Current Summation"}

For completeness, we shall discuss the possibility that the suppression of critical currents might be a spurious effect associated with the summation of an initial supercurrent and an externally injected current, as recently suggested in Ref. [125], and then comment on the preliminary data taken on patterned F-I-S heterostructures. One may conjecture that as the externally injected current from the manganite underlayer enter the superconductor uniformly in a direction transverse to the $J_{s}$ measurement current in the superconductor, this injected current would affect the measurements of $J_{c}^{+}$and $J_{c}^{-}$values differently due to the spatial variation in the local current density inside the superconductor, as illustrated in Fig. 6.3. That is, one might assume that $J^{+}(y)=J_{s}+(y / L) J_{i n j}$ and $J^{-}(y)=-J_{s}+(y / L) J_{i n j}$, where $L$ is the length of YBCO along the $J_{s}$ direction, $J_{s} \| \hat{y}$, and further conjecture that the I-V characteristics of the entire superconductor would be solely determined by small resistive regions in the superconductor. More specifically, an apparent suppression of the measured $J_{c}$ would be expected because $J_{c}^{+}$would be reduced by $J_{i n j}$ through direct addition, while $J_{c}^{-}$would be unaffected and would remain the same as $J_{c 0}$ [125]. However, upon closer scrutiny, such a hypothetical scenario lacks merit for a number of reasons.

Empirically, all existing data derived from the as-grown and patterned devices can unambiguously rule out the current-summation scenario as the explanation for our obser- 


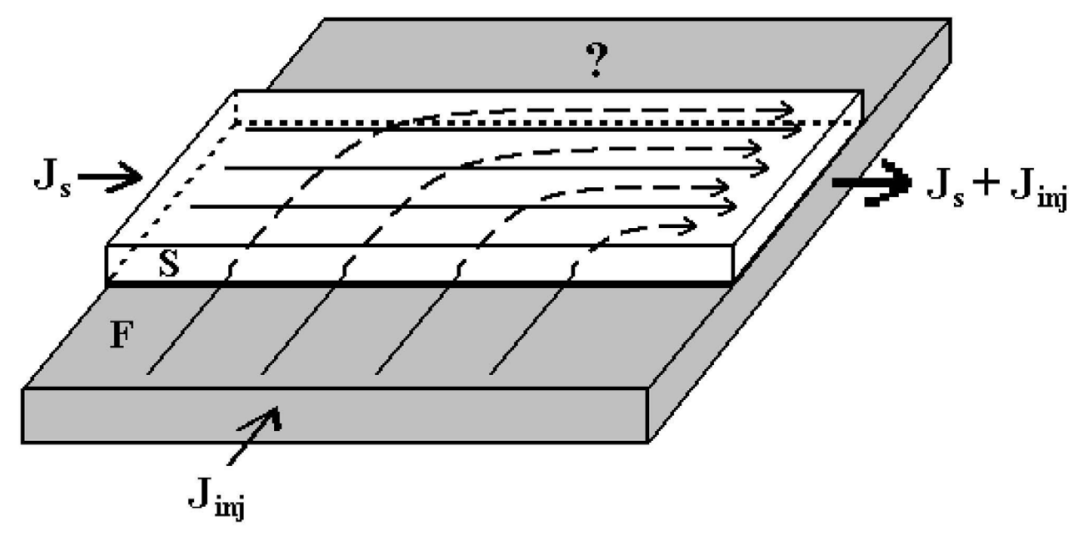

Figure 6.3: Hypothetical current flow patterns in the YBCO layer of the F-I-S heterostructure under external current injection from the CMR film [116].

vation. First, had the summing of currents been the dominating cause for the suppression in $J_{c}$, we would have found no change in $\left|J_{c}^{-}\right|$and significant suppression only in $\left|J_{c}^{+}\right|$. However, such behavior has never been observed in any of our as-grown or patterned samples. Second, this two-dimensional geometrical effect would have resulted in a constant efficiency $\eta=1 / 2$, for all $J_{i n j}$ at all temperatures below $T_{c}$, and for all samples, regardless of the sample types (i.e., F-I-S vs. N-I-S) and the constituent layer thickness. This clearly is contradicted by the experimental data shown in Figs. 5.12(a) and (b) for the as-grown heterostructures where $\eta$ varies significantly with $T$ and $J_{i n j}$. In addition, for as-grown samples, no appreciable suppression in $J_{c}$ could be detected in the F-I-S devices with either a thicker superconducting layer $(d=160 \mathrm{~nm}$ ) (see Fig. 5.11(b)) or a thicker insulating barrier (10 nm) [53] at all temperatures except very near $T_{c}^{-}$, implying $\eta \ll 1 / 2$. Similarly, no discernible $J_{c}$ suppression could be found in the control N-I-S heterostructure with $d=100 \mathrm{~nm}$ [53], implying $\eta_{n} \sim 0$ for a wide range of temperature. Third, the simple current-summation scenario would assert that $J_{c}=0$ if $J_{i n j} \geq 2 J_{c 0}$ for all heterostructures 
at all temperatures, which is at odds with the data shown in Fig. 5.6(b) for a control N-I-S sample. As discussed previously, such finding in the N-I-S samples is a clear revelation of the uncertainties in the injected quasiparticle momentum [45]. Fourth, we note that the experimental results by Vas'ko et al. [51] have demonstrated that the suppression $J_{c}$ in spin injection devices is independent of the direction of current injection relative to the supercurrent, which further corroborate the notion that the positions of external electrodes do not provide well-defined supercurrent distribution within a superconductor. Finally, the scanning tunneling spectroscopic (STS) studies of the YBCO layer in both F-I-S and N-I-S heterostructures in this group had demonstrated distinct changes in the quasiparticle DOS only under spin injection, as shown in Fig. 6.4. The STS experiments were performed with $J_{i n j} \geq 0$ and $J_{s}=0$ at all times; hence, no complications from current addition were involved. Thus, we conclude that all experimental data to date clearly rule out the possibility of current-summation as an alternative explanation for $J_{c}$ suppression in perovskite F-I-S devices.

From the theoretical viewpoints, the current-summation scenario assumes that the injected quasiparticles follow a well defined current path, which immediately turn after entering the superconductor, flow toward the common-ground terminal, and exit the superconductor after aggregating at that end of the superconductor, as depicted in Fig. 6.3. In other words, although the incident quasiparticle momentum was along the $c$-axis, only the final momentum parallel to the direction of the supercurrent in the $\mathrm{CuO}_{2}$ planes was considered relevant. Such approach is unjustified for the following reasons. First, the hypothetical geometric effect for a partially varying total current density in the superconductor 

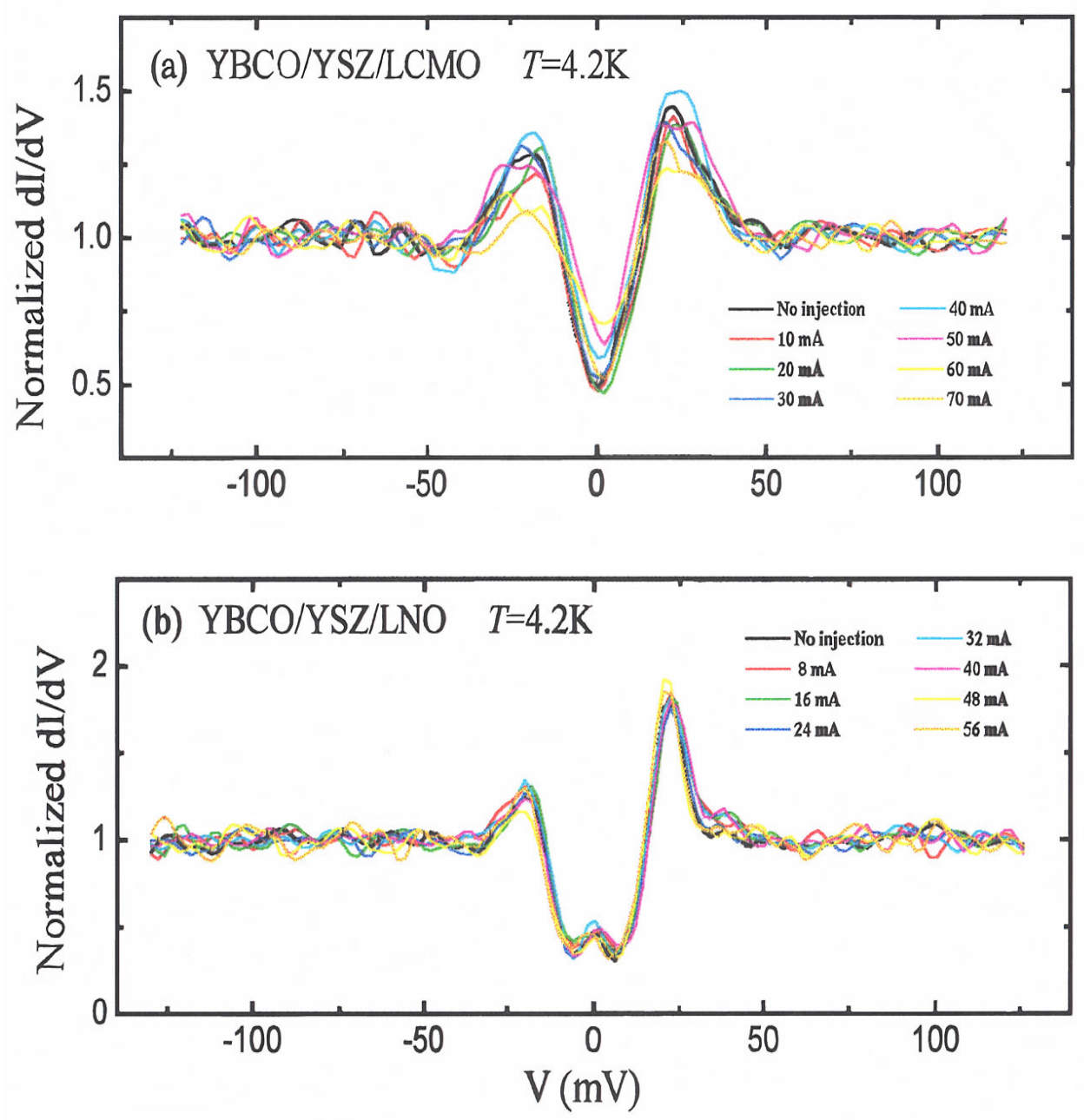

Figure 6.4: Scanning tunneling spectroscopic (STS) studies of the YBCO layer in both F-I-S and N-I-S heterostructures. Only under spin-polarized quasiparticle injection do the energy peaks experience pronounced smearing [55]. 
would have resulted in a phase gradient in the order parameter throughout the superconductor. Such a gradient would have incurred phase slippage and vortex formation in the superconductor, and the interaction of the non-uniform currents with vortices would tend to redistribute the currents more uniformly to minimize the phase gradient. Thus, the real current distribution inside the superconductor is expected to deviate from the direct sum of currents. Second, the dynamic nature associated with the initial interaction of the injected quasiparticles with the superconductor plays a very important role in determining the nonequilibrium superconducting properties, such as the overall quasiparticle energy and the DOS. These important processes such as the quasiparticle redistribution and pair recombination could not be neglected unless the quasiparticle relaxation times were sufficiently short so that the corresponding characteristic lengths were much smaller than the sample dimensions. However, the in-plane spin relaxation time could range from $10^{-4} \mathrm{~s}$ at $\left(T / T_{c}\right) \ll 1$ to $10^{-6}$ s at $\left(T / T_{c}\right) \rightarrow 1$, so that the in-plane spin relaxation length was on the order of $10^{-4} \sim 10^{-3} \mathrm{~m}$, comparable to the device dimension. The nonequilibrium effect of spin-polarized quasiparticles appeared to be long-range at all temperatures in F-I-S samples with thin YBCO, and therefore cannot be neglected. Third, the current-summation scenario ignores the dependence of quasiparticle transmission across interfaces on the degree of spin polarization, the quasiparticle energy, the no double occupancy constraint, and the interface properties. Such simplification is neither theoretically rigorous nor empirically compatible with experimental data.

In an earlier study of an N-I-S heterostructure [126, 127], a suppression of $J_{c}^{+}$ obtained was attributed to the effect of current summation in Ref. [125]. Upon closer 
inspection of the experiments, it can be ascertained that the critical current had been determined only in one supercurrent direction $\left(J_{c}^{+}\right)$, while the reversal of polarity was done to the injection current in the measurements. In reality, the experimental procedure in Ref. [126] gave rise to a branch imbalance effect associated with the injection of charged quasiparticles [45], as discussed in Chapter 2. That is, reversing the polarity of the injection gate current actually changed the injected quasiparticles from predominately electron-like to hole-like in character, or vice versa. It is known that a complete description for the branch imbalance effect must include studies of both polarities of the bias voltage [45]. Similarly, showing the full $J_{c}$ behavior requires the mapping of both $J_{c}^{+}$and $J_{c}^{-}$values, but no results for $J_{c}^{-}$were reported in Ref. [126]. Therefore, the current summation conjecture remains unsubstantiated, contrary to the assertion in Ref. [125].

On the magnitude of the efficiencies $\eta$ associated with both F-I-S and N-I-S heterostructures, we note that they are generally small except at low temperatures in the F-I-S. This is not entirely surprising because the YBCO superconductor is known to have $d$-wave pairing symmetry, which is gapless along the nodal directions. The pre-existence of thermally excited quasiparticles diminishes the significance of those injected externally. Only in the low temperature regime, where the nonequilibrium effects become significant, does one observe larger $\eta$ in the spin-polarized quasiparticle injection. Similar findings of small efficiencies under injection have been confirmed by a different experimental technique through magnetization measurements of YBCO films [128].

Finally, based on the phenomenological analysis of our experimental data, we remark that the bulk nonequilibrium effects in perovskite F-I-S and N-I-S heterostructures 
appear to be conceptually consistent with the general descriptions for quasiparticles. In other words, there is no obvious need to invoke spin-charge separation in the superconducting state to account for the spin and charge transport behavior in the cuprates. 


\section{Chapter 7}

\section{Basics in Thin-Film Processes}

In this chapter, basic principles of several thin-film processing, including evaporation, sputtering, ion-beam etching and reactive ion etching, are described. These processes are essential for thin-film deposition, removal and patterning, and are the bases for many of the procedures referred to in the F-I-S and N-I-S device fabrications described later in Chapter 8.

\subsection{Evaporation Processes}

An evaporation system is generally used as a low-energy deposition of thin films. Typically it consists of an evaporation source, which can be a thermally heated crucible, an electron or a laser beam, to vaporize the desired material from condensed phase to vapor phase. The substrates are located at a suitable distance facing the evaporation source. The substrate holder can be heated and/or biased to a desired potential using a dc/rf power

supply. Evaporation is carried out in vacuum in a pressure range of $10^{-5}-10^{-8}$ Torr, where 
molecular flow applies. The mean free path $(\mathrm{MFP})$ in this pressure range $\left(5 \times 10^{2}\right.$ to $\left.10^{5} \mathrm{~cm}\right)$ is much longer than the source-substrate distance, therefore, the evaporated atoms undergo essentially collisionless line-of-sight transport prior to condensation on the substrate, thus a thickness buildup occurs directly above the source and decreases dramatically away from it. Viscous flow occurs when a higher pressure, typically 20-120 mTorr, is used. The gas-scattering evaporation technique, operating in this pressure range, introduces into the chamber a gas such as argon to reduce the MFP, thus causing the vapor species to experience multiple collisions during transport from the source to the substrate. This method produces a more uniform thickness of coating on the substrate.

\subsubsection{Theory and Mechanisms}

The transition of solids into gaseous state can be considered to be an atomistic phenomenon. The theory of vacuum evaporation involves thermodynamics and depends on the understanding of evaporation rates, source-container reactions, and stability of compounds.

The rate of evaporation is given by the well-known Hertz-Knudsen equation [129],

$$
\frac{d N_{e}}{A_{e} d t}=\alpha_{v}\left(2 \pi m k_{B} T\right)^{-1 / 2}\left(p^{*}-p\right)
$$

where $d N_{e} / A_{e} d t$ is the number of molecules evaporating from a surface area $A_{e}$ in time $d t$, $\alpha_{v}$ is the evaporation coefficient, $m$ is the molecular weight, $p^{*}$ is the equilibrium vapor pressure at the evaporant surface, and $p$ is the hydrostatic pressure acting on the surface. It is worth noting that $\alpha_{v}$ is highly dependent on the condition of the evaporant surface and ranges from very low values for dirty surfaces to unity for clean surfaces. 
For normal deposition rates of $100-1000 \mathrm{~nm} / \mathrm{min}$ at a source-substrate distance of $\sim 20 \mathrm{~cm}$, the vapor pressure should be about $10^{-2}$ torr. The source temperature is usually adjusted to give this value of the vapor pressure.

The directionality of evaporating molecules from an evaporation source is given by a cosine law. In the ideal case of deposition from a uniformly emitting point source onto a plane receiver, the rate of deposition varies as $\cos \theta / r^{2}$, where $r$ is the radial distance of the substrate from the source, and $\theta$ is the angle between the radial vector and the normal to the substrate direction. Given that $t_{0}$ and $t$ are the thicknesses of deposition at the substrate vertically below the source at a distance $h$, and at a horizontal distance $x$ from the vertical, respectively, the deposition distribution should be

$$
\frac{t}{t_{0}}=\frac{1}{\left[1+(x / h)^{2}\right]^{3 / 2}}
$$

For evaporation from a small area onto a parallel plane receiver, the proportionality changes to $\cos ^{2} \theta / r^{2}$, and the corresponding thickness distribution is given by

$$
\frac{t}{t_{0}}=\frac{1}{\left[1+(x / h)^{2}\right]^{2}}
$$

In either case, the deposition decreases by about $10 \%$ for $x=h / 4$.

One way to overcome the problem with the variation in thickness of the deposit on a flat surface is to move the substrate for a random sampling of the vapor flux [129, 130]. Another is to use multiple sources to enlarge the region of uniformity in the deposition $[129,130]$. 


\subsubsection{Evaporation Apparatus}

The evaporator typically includes a deposition chamber, a vacuum pump, substrate holder fixture and heaters, evaporation sources, and deposition rate monitor. Some important features for each component in the evaporation system are discussed in following.

\section{Vacuum Chamber}

A simple bell jar is commonly used to enclose the vacuum chamber, but it is necessary to provide radiation shields around the evaporation source to prevent excessive heating of the glass bell jar. For an evaporation process using electron beam guns, it is preferable to use a stainless steel bell jar. More complex systems, involving multiple loading/unloading chambers attached to the deposition chamber via manifolds with isolation high-vacuum valves, have also been designed. It may be necessary to add water cooling to the bell jar chamber designs, in the case of high-power operations.

\section{Vacuum Pumping System}

The gas loads in evaporation processes are quite high because of outgassing from chamber walls promoted by the heat load from the evaporation source, substrate heating, etc., and even more so for high deposition rate situations. Therefore, the pumping system is usually based on a diffusion pump, backed with a mechanical pump. For ultra-high purity, low rate of deposition and low heat flux conditions, ion pumps backed with turbomolecular

or cryosorption roughing pumps are employed, as very low base pressures in the range of $10^{-9}-10^{-10}$ Torr are required. 


\section{Substrate Holder and Heaters}

The design of the substrate holder is primarily determined by the deposition uniformity criterion, as well as the shape of the objects to be coated. It may be very simple for a flat substrate, or it can incorporate quite complex motions to obtain uniform thickness distribution over a large substrate or a large number of small pieces, such as lenses and silicon wafers, etc.

\section{Evaporation Sources}

Evaporation sources are classified in terms of the mode of heating employed to convert the solid target to the vapor phase. Thus one can consider different types of sources including resistance, induction, arc, electron-beam, and laser. The choice is dictated by the material to be evaporated, as well as the desired rate of deposition. To avoid contamination and to ensure the purity of the deposit, it is necessary to use support materials for the target with negligible vapor pressure and dissociation pressure at the operating temperature. In addition, the possibilities of chemical reactions and alloying between support materials and the target material should be considered and minimized. Chemical reactions tend to produce volatile contaminants, such as oxides, which could be incorporated into the film as impurities. Alloying reduces melting point and could result in the destruction of the source itself.

\section{Resistance-Heated Sources}

Resistance-heated sources of various types, shapes, and sizes can be easily constructed; some examples are shown in Fig. 7.1. These sources are generally made of high 

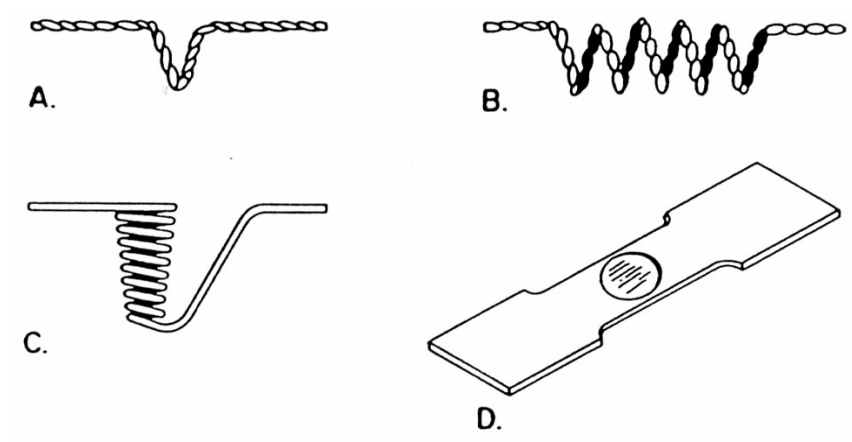

Figure 7.1: Resistance-heated sources: a) hairpin; b) wire helix; c) wire basket; d)dimpled foil [131].

melting-point, high vapor-pressure refractory metals such as tungsten, molybdenum, tantalum, or platinum, in order to avoid contamination. Iron or nickel can be selected in the event that evaporation of target occurs below $1000{ }^{\circ} \mathrm{C}$.

One single source is typically used for low capacity evaporation. Multiple sources in a series or parallel configuration can also be used to increase the evaporation rate as well as the coating region and uniformity. A thickness monitor with feedback loop to control the input power of the source can also be used to achieve controlled and uniform evaporation.

\section{Electron Beam Sources}

A typical electron gun system includes a cathode and an anode. The electrons are emitted from the cathode and are accelerated across the potential drop (ranging from a few kilovolts to about $30 \mathrm{kV}$ ) between the cathode and the anode. There are two modes of electron generation in electron beam guns: one is thermionic and the other is via plasma. In thermionic guns, the electrons are produced by resistive heating of a wire made up usually of tungsten or tantalum. This type of guns operate at pressures below $1 \mathrm{mTorr}$. Pressures above that level tend to cause scattering of the electron beam and shorten the cathode 
life because of erosion from ion bombardment. In plasma guns, proper implementation of electrical potential can extract electron from the ionized plasma in the cathode. In general, these electron beam sources offer the advantage that they provide high power density, and a wide range of control over evaporation rate. Moreover, with the use of water-cooled crucible, the electron beam only heats the surface of the evaporant to a high temperature, so reactions with the crucible leading to film contamination are effectively eliminated.

\section{Pulsed Laser Beam Evaporation (Laser Ablation)}

Laser beams have been used to melt and evaporate the material, with the wavelength of the laser chosen by the absorption characteristics of the material used in the evaporation. Often, pulsed laser beams are employed to reach the high power density output required for evaporation. Generally, the flexibility of pulse width, repetition rate, and pulse intensity selection depend on the specific application of the deposition. Pulsed laser evaporation, also known as laser ablation, has been the leading technique in the synthesis of high temperature superconductor films in recent years. For evaporation of the $\mathrm{YBa}_{2} \mathrm{Cu}_{3} \mathrm{O}_{7-\delta}$ films, Nd-YAG lasers at $532 / 242 \mathrm{~nm}$, with pulse energy in the range of 0.2-1 $\mathrm{J}$ and repetition rate of $10-30 \mathrm{~ns}$, have been used extensively.

Even with the success of synthesis of high-quality compound films, it is worth noting some of the drawbacks associated with laser evaporation. First, a complicated transmitting and focusing mechanism is needed to direct the beam from the laser source located outside the vacuum system onto the target placed inside the system. A window material that is able to efficiently transmit the wavelength band of the laser must be setup in a way that it is not rapidly covered up by the evaporant flux. Second, it is sometimes 
difficult to match a laser with a wavelength compatible with the absorption characteristics of the target material. Third, the efficiency of energy conversion using the laser beam is very low.

\subsection{Sputter Deposition Processes}

Sputtering deposition is a plasma-based process, which differs from evaporation deposition in that the plasma process is not thermal, and the dynamics of film formation are not describable by equilibrium thermodynamics. A simplified cross section of a sputtering system is represented in Fig. 7.2. Typically, the target material to be deposited is connected to a negative voltage supply. The substrate is held facing the target, with the holder either grounded, floating, biased, heated, cooled, or some combination of these. A gas is introduced to provide a medium in which a plasma can be started and maintained. The gas pressure ranges from a few to about 100 mTorr. The most common gas used is argon.

When the plasma is initiated, positive ions strike the target plate and remove mainly neutral target atoms by momentum transfer, and these target atoms condense into thin films on the substrate at a high incident energy (typically 10 to $100 \mathrm{eV}$ compared to $0.5 \mathrm{eV}$ for evaporation) and usually with good adhesion. There are also other particles and radiation, such as secondary electrons and ions, desorbed gases, x-rays, and photons, produced at the target, all of which may affect film properties.

Sputter sources for film deposition can be categorized in two ways: glow discharge (diode, triode, and magnetron) and ion beam. The focus of this discussion will be on magnetron sources, specifically circular planar magnetrons (used in the experiments), as 


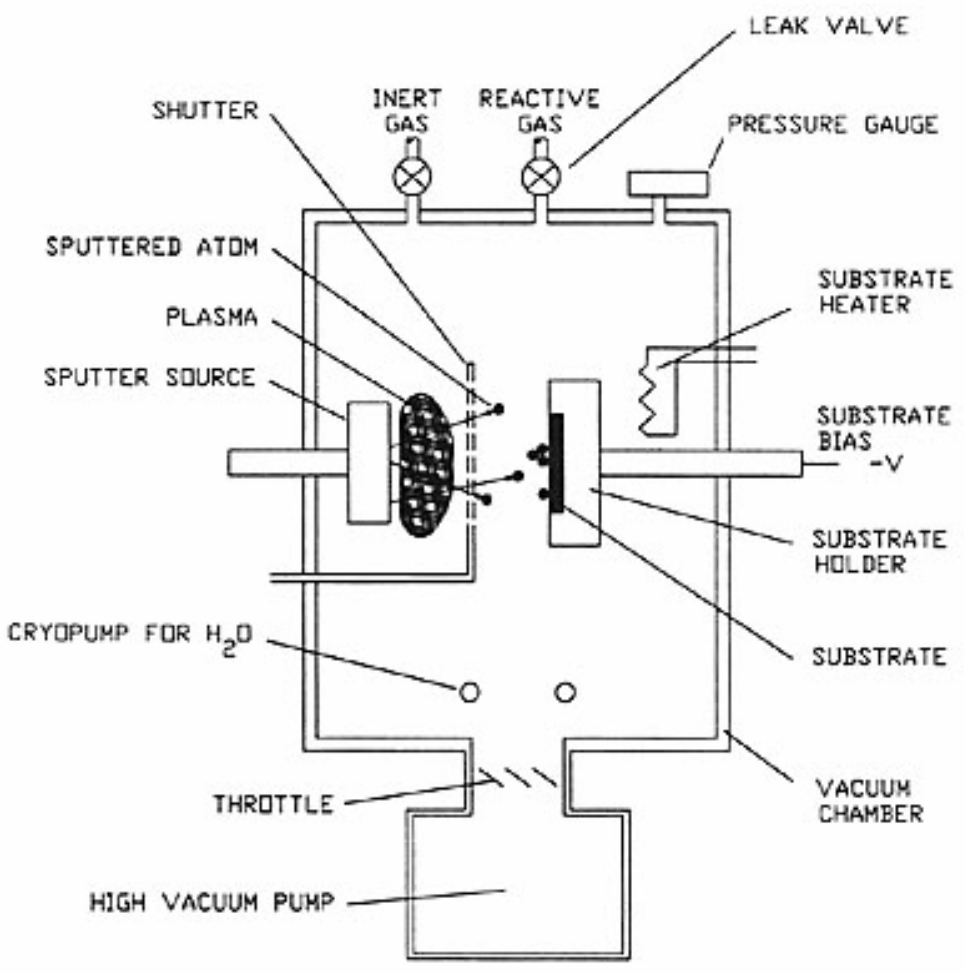

Figure 7.2: General schematic of a sputtering system [131].

a complete treatment of all sources is beyond the coverage of this section. However, the basic mechanism by which the particles are ejected through momentum exchange between energetic particles and surface atoms on the target remains the same for the different sources.

\subsubsection{Processing Plasma}

Processing plasmas are produced and driven by external power supplies, which range in frequency from dc up to $\sim 10 \mathrm{GHz}$, and in power up to $30 \mathrm{~kW}$. One method used to create and sustain plasmas is by applying a high voltage to a set of metal electrodes (cathode and anode) within the discharge chamber. Electrons are emitted as secondaries 
from the ion bombardment of the negatively powered cathode. The secondary electrons are accelerated away from the cathode towards the anode, and in doing so, they gain enough energy to cause the ionization of neutral gas atoms. These ionized atoms in turn bombard the target and release more secondary electrons in an avalanche process.

There are a number of inelastic collision processes that occur in the plasma. The most important process is ionization:

$$
e^{-}+A r \rightarrow A r^{+}+2 e^{-}
$$

This reaction contributes an additional electron along with the ion. A doubly ionized atom by the removal of an extra electron is possible, but that requires significant additional energy on the part of the incident electron.

Another very common process within a plasma is the excitation of an atom or an ion into an excited state:

$$
e^{-}+A r \rightarrow A r^{*}+e^{-}
$$

After a very short lifetime in the excited state, the additional energy carried is released through a radiative decay and results in the emission of a photon. This process is responsible for the "glow" that is characteristic of all plasmas.

Sometimes, an excited state that has a long lifetime, of the order of milliseconds, can populate the plasma. During that lifetime, the excited atom may have a collision with a ground state atom to cause either excitation or ionization of that atom. Theses processes, known as Penning processes, can be described as

$$
X^{*}+Y \rightarrow X+Y^{*} \text { (excitation) }
$$




$$
X^{*}+Y \rightarrow X+Y^{+}+e^{-} \text {(excitation) }
$$

where $\mathrm{X}$ and $\mathrm{Y}$ are arbitrary species.

\subsubsection{Plasmas in the Presence of E and B Fields}

A moving charge in a magnetic field $\mathbf{B}$ is subject to a force $\mathbf{F}=q \mathbf{v} \times \mathbf{B}$, where $q$ is the charge on the particle, and $\mathbf{v}$ is the velocity. For a charged particle moving at right angles to a magnetic field, this force will cause the particle to move in an orbital path, with orbit radius

$$
r=\frac{m v_{\perp}}{q B}=\frac{\sqrt{2 m(\mathrm{KE})}}{q B}
$$

where $m$ is the mass of the particle, $v_{\perp}$ is the component of the velocity perpendicular to the magnetic field, and KE is the kinetic energy of the particle. The component parallel to the magnetic field is unaffected, so the resulting path of the charged particle is helical. It is apparent that particles with either high mass or high energy will have large orbits. In general, the electron orbits in most plasmas will be small with respect to the dimension of the chamber, while the ion's orbit will be larger than the sputtering system and can usually be ignored.

In the case when the electron is moving in both a magnetic $\mathbf{B}$ and an electric $\mathbf{E}$ field, in particular if they are perpendicular to each other, it experiences a constant force along the direction of the electric field, in addition to the orbital motion from the magnetic field. The electric force has the effect of slowing down the electron when it is moving in the semicircle with an antiparallel component relative to $\mathbf{E}$ in its motion. The result is that the orbit becomes effectively smaller. When the electron is moving in the other semicircle 


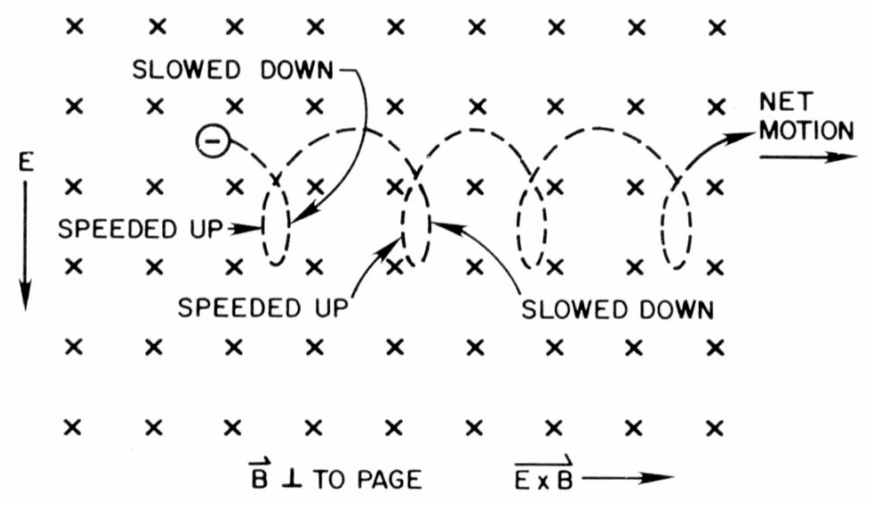

Figure 7.3: The motion of an electron in the presence of perpendicular electric and magnetic fields. Here the $\mathbf{E}$ field is vertical, and the $\mathbf{B}$ field is in and out of the page [131].

with a parallel component relative to $\mathbf{E}$, the electron is accelerated and its effective radius increases. The net effect is that the cyclical motion adds an effective drift in the direction perpendicular to both the electric and magnetic fields (see Fig. 7.3). This is known as the $\mathbf{E} \times \mathbf{B}$ drift, with a drift velocity

$$
V_{\mathbf{E} \times \mathbf{B}}=E / B
$$

Again, because the orbits are much too large for the ions in the plasma, this type of effect is usually of interest only for election motions.

\subsubsection{Circular Planar Magnetrons}

With proper orientation of the electric and magnetic fields, the $\mathbf{E} \times \mathbf{B}$ drift paths can be configured to form a closed loop. Therefore, instead of losing the drifting electrons at one end of the plasma, the drifting electrons, as illustrated in Fig. 7.4, can recirculate and be effectively trapped near the cathode in a drifting closed loop current. This device is known as a magnetron. 


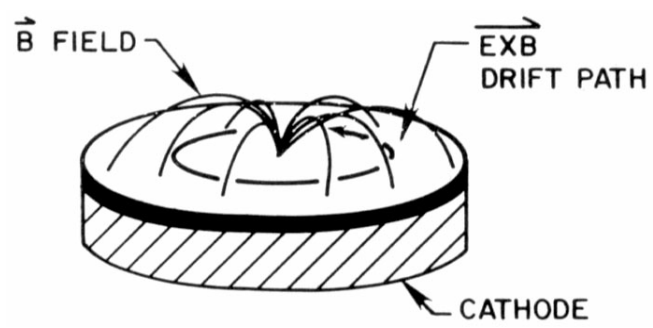

Figure 7.4: The shape of the magnetic field of a circular magnetron cathode and the resulting plasma drift path [131].

The circular planar magnetron, as shown in Fig. 7.5, consists of a water-cooled cathode surface back by an array of magnets. The poles of the magnets are oriented such that the center axis of the device is the first pole, and an annular ring at the edge of the cathode is the second. Near the poles, the magnetic field lines intercept the cathode surface, but are approximately parallel to the surface elsewhere in general. By orienting the magnetic field to be radial, there is an annular path in which the $\mathbf{E} \times \mathbf{B}$ drift is parallel to the cathode surface and forms a closed path. The secondary electrons emitted from the cathode are constrained to this annular loop, resulting in increased ionization and a dense plasma. This electron confinement significantly increases the efficiency and, as a result, a magnetron can operate at low pressures and low voltage. The magnetrons typically has a magnetic field of about 50 to $500 \mathrm{G}$ parallel to the target surface. This magnetic field at these strengths does not directly affect the ion motion, but the ions do move with the electrons because of electrostatic attraction, which keeps the plasma neutral.

The current density at the cathode of a magnetron is the highest where the magnetic field lines are tangent to the surface of the cathode, thus, the erosion of the target is nonuniform. In fact, poor utilization of the target material is a disadvantage of the planar 


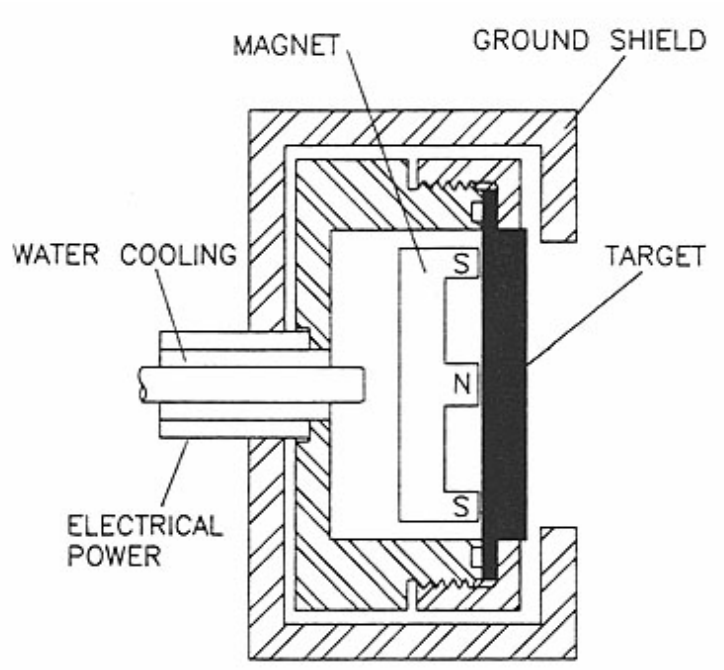

Figure 7.5: A cross section of a planar magnetron sputter source [131].

magnetron. Uneven target usage can be a severe problem in the case of magnetic targets. If permeability of the target is high, magnetic flux leakage is concentrated at narrow regions on the target surface, and a narrow erosion track results.

\subsubsection{Sputtering Target}

It is possible to use any kind of material as a sputtering target, but the highest purity work should be done with very dense targets. Targets that are sintered, hot-pressed, powdered, and liquid have shown to introduce considerable gaseous contamination. One major contaminant appears to be oxygen absorption onto the surface during hot pressing, which can be severe depending upon the application and the tolerance of the intended film properties.

Pre-sputtering of targets is performed to clean and equilibrate target surfaces before initiating film deposition by a blocking shutter usually located close to the substrate. 
For pure metal targets, surface oxides are removed and the system is outgassed after the pre-sputtering period. In addition, the target surface is brought to thermal equilibrium. Oxides tend to have high secondary electron emission ratio, so during pre-sputtering, the discharge current is initially elevated and gradually decreases as the surface layer is sputtered off and any background gaseous contaminant, especially $\mathrm{H}_{2} \mathrm{O}$, is being broken down. When monitoring the discharge current, pre-sputtering can be terminated when it levels off to a constant value.

\subsubsection{The Pump}

Sputter coaters traditionally employ a diffusion pump, an oil-sealed mechanical pump, and a liquid nitrogen cryotrap. The diffusion pump provides high pumping speed and high continuous gas loads at low cost. A turbomolecular pump, with similar performance as the diffusion pump, can also be used. Its advantages are its tolerance to sudden gas overloading and oil/grease backstream.

\subsubsection{Sputtering Yield}

The sputtering yield is defined as the number atoms ejected from a target surface per incident ion. Although this is the most fundamental parameter of the sputtering process, not all of the surface interaction phenomena that contribute to the yield is completely understood. There is a threshold for sputtering that is approximately equal to the heat of sublimation. In the energy range typical of sputtering processes $(10-5000 \mathrm{eV})$, the yield increases with incident ion energy, and with the mass of the incident ion. The sputtering yield determines the erosion rate of the targets and as a result, determines the deposition 
rate of the sputtered films primarily.

\subsection{Ion Beam Etching}

Ion beam etching (IBE) is a dry-etch process that employs a broad-beam ion source, which generates high beam currents (tens of milliamperes to several amperes) at low ion energies. The large beam currents are necessary for high processing rates, and the low ion energies are important for minimizing damage to the etched surface. Ion beam processing is normally used on etch-resistant materials difficult or impossible to process with older processes, like rf diodes or plasma etching. It also offers greater control of background pressure, ion energy, direction and arrival rate.

The most common type of broad-beam ion source is the gridded type, which is shown schematically in Fig. 7.6. The ions are accelerated through the apertures in the tightly spaced grids. The accelerator grid is negative of ground and keeps electrons from backstreaming. The ions speed up toward the negative accelerator, but slow down quickly to a velocity specified by the beam voltage after leaving the grid.

\subsubsection{Ion Generation}

The ions in the IBE system are generated in a discharge chamber, in which the atoms or molecules of the gas to be ionized are bombarded and ionized by energetic electrons. These electrons are emitted by a cathode, usually a hot filament, and reach the anode after

collisions. A magnetic field is used to contain the energetic electrons and enhance the ionization, since the chamber is at a low gas pressure (in the $10^{-4}$ Torr range). 


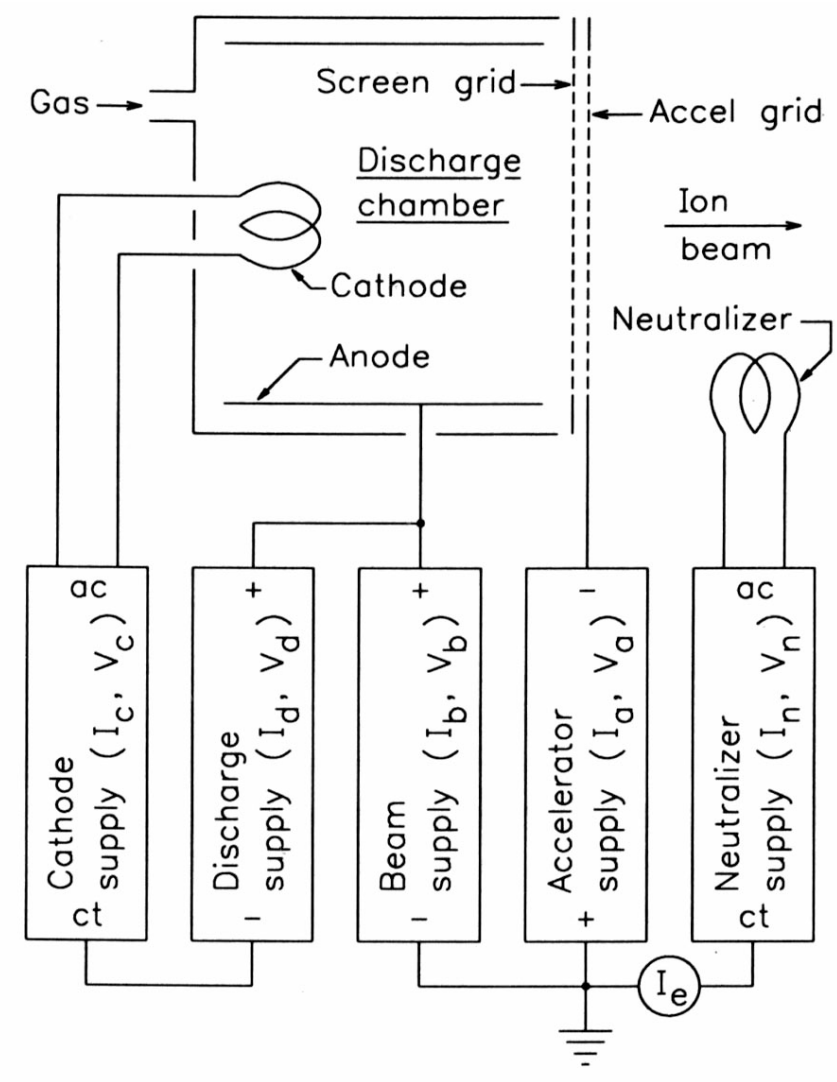

Figure 7.6: Schematic diagram of a gridded ion source [131].

The energetic electrons, together with low-energy background electrons and ions in the discharge, form a plasma. The potential of this plasma is within several volts of the anode potential. The electrons emitted from the cathode acquire an energy that corresponds to the voltage on the discharge supply. It is important to operate the discharge at a low voltage (typically $40 \mathrm{~V}$ or less) to minimize the production of doubly charged ions. This reduces the contamination from the discharge chamber. Even though etching often is a self-cleaning process where contamination is etched away with the material being etched, some contamination is actually driven deep into the etched surface by hard collisions. The 
production of doubly charged ions depends primarily on the discharge voltage. Sufficiently energetic electrons can remove two electrons in a single collision. As the discharge voltage is increased, both the collision energy and the percentage of doubly charged ions are increased. The threshold for sputtering of most chamber materials is in the $20-40 \mathrm{eV}$ range, so a singly charged ion with energy less than $40 \mathrm{eV}$ will cause minimal sputtering. However, at 50-60 V, a significant number of doubly charged ion species will be ionized. Given that charged ions close to the cathode-potential surfaces will be attracted to and collide with these surfaces at an energy equal to about the discharge voltage for singly charged ions, and about double the value for doubly charged ions, these doubly charged ions could strike the cathode at energy in the range of $100-120 \mathrm{eV}$.

\subsubsection{Ion Acceleration}

The ions from the discharge plasma first pass through apertures in the screen grid as they respond to the attraction toward the negatively biased accelerator grid. They pass through the accelerator grid and go on to form the ion beam. If an operating voltage exceeds a maximum beam current, some of the energetic ions will spread out and strike the accelerator grid, rather than passing through it. This will result in both contamination of the target with accelerator grid material and rapid erosion of the accelerator grid. Also, to reduce accelerator erosion, it is recommended to keep the accelerator voltage to the minimum necessary to prevent backstreaming of electrons, usually at $15-20 \%$ of the beam voltage, and to reduce the background pressure.

The most common grid materials are pyrolytic graphite and molybdenum. Graphite is a frequent choice for small ion sources and for some reactive gases, except for oxygen. 
Molybdenum is more suited for larger ion sources because molybdenum is much stronger and stiffer than graphite.

\subsubsection{Beam Neutralization}

The ions in the ion beam have positive charge. It is necessary that electrons be added to "neutralize" the ion beam. In the case of an insulator target, a positive charge will accumulate on the insulator surface until either an electrical discharge occurs or additional ions are repelled and prevented from bombarding the target. When a conducting surface is being etched, the need to charge-neutralize the ion beam is still there. If there were no electrons present to balance the positive charge in the beam, there is usually enough ions in the beam that the mutual repulsion of positive ions would cause a divergence of the beam in all directions. The neutralization does not involve the process of recombination

of electrons with the beam ions. It simply permits an equal number of electrons and ions to reach the etched surface.

When a hot filament, heated by an ac neutralizer supply, is used as a neutralizer, it is immersed in the ion beam in order to provide adequate electrical coupling to the beam. However, being in the path of the ion beam, the neutralizer is subject to sputtering by the ions. To avoid this type of contamination, a hollow-cathode neutralizer can be used, which is located just outside of the ion beam and can still sufficiently couple to it.

\subsubsection{Process Control}

The attractive feature of ion-beam etching is the ability to independently control the ion energy, ion current density, ion direction, and background pressure to obtain desired 
results. In other dry-etch processes, it is typical for changes in ion energy to be coupled with changes in ion current density. Systems with increased ion current capacity have allowed the use of ion energies under $800 \mathrm{eV}$. It can be shown that the optimum energy for removal of material by physical sputtering is under $500 \mathrm{eV}$. In addition, reduced ion energy also decreases surface damage from ion etch. At the same time, the corresponding reduction in photoresist damage makes removal easier after the pattern etch.

The independent control of pressure in ion-beam etching results from the fact that the ion generation is located in the discharge chamber at a distance from the target. The background pressure is generally in the low $10^{-4}$ Torr range.

\subsection{Reactive Ion Etching}

Reactive ion etching is commonly performed in rf glow discharges. A reactive gas species, such as $\mathrm{O}_{2}, \mathrm{CF}_{4}$, or $\mathrm{SF}_{6}$, is added to a discharge chamber with induced rf diode plasma. The gas molecules are broken into radicals or become ionized in the plasma, and may then be accelerated to the various electrode surfaces. Samples are placed on these electrodes and thus exposed to the flux of ions from the plasma, as well as the isotropic flux of gas species present within the chamber.

During reactive ion etching, gaseous species from the plasma react with surface atoms to form compounds or molecules. These species then lift off from the surface thermally, as a result of ion bombardment or high vapor pressure. The reaction rate is influenced by the production rate of reactive species in the plasma, by the surface reaction rate, or by the emission rate of product molecules. Often, ion bombardment is very useful in main- 
taining the anisotropy of the etch process. It tends to clean the surface, to stimulate the reaction itself, and to detrap the product molecules. 


\section{Chapter 8}

\section{Microfabrication/UV Lithography}

So far, the F-I-S samples discussed in the previous chapters have been as-grown devices where the YBCO bridge are made during the growth process via a simple shadowmasking technique. As discussed in the previous chapter, besides the technical difficulty in using the pulse-current measurement, such imprecise and large-scale samples complicate further quantitative analyses. Therefore, YBCO bridges of dimension ranging from 100 $\mu \mathrm{m}$ to $1 \mu \mathrm{m}$ are designed in a photomask pattern, which will be used in conjunction with lithographic techniques to make devices corresponding to those dimensions.

Because YBCO superconductor is a highly sensitive material, it is important to devise a set of procedures most beneficial to maintaining the superconductivity. Patterned devices were fabricated at Micro Devices Laboratory of JPL, with assistance from Dr. Alan Kleinsasser and Dr. Yonggyu Gim, and at Caltech. Sec. 8.1 will cover the procedures of photolithography in general terms. In Sec. 8.2, specifics of the microfabrication pro-

cedures, which were derived from a template written by Dr. Jeffrey Barner of JPL and 
were subsequently refined through the use of vendor (Clariant Corp.) documentation for the photoresist and further process development, will be discussed. This is followed by details in the measurement technique and preliminary results from the fabricated devices. A summary of the results concludes this chapter.

\subsection{UV Lithography}

\subsubsection{Sample Preparation}

The sample should first be inspected visually, noting any surface imperfections. The back of the substrate is usually covered with silver paint, which is applied during sample growth for adhesion; this silver coating should be scraped off using a \#10 scalpel to prevent possible shorting along the edges. A diamond scribe is used to permanently label the back of the samples to avoid confusion when multiple samples are processed simultaneously. The cleaning procedure for these samples involved standard solvents in the order of acetone, isopropanol, and ethanol, in ultrasound. If necessary, xylenes may be used during cleaning. The sample should be free of organic contamination and excessive physically adsorbed moisture. A dehydration bake at $105{ }^{\circ} \mathrm{C}$ for 1 minute is recommended.

\subsubsection{Application of Resist}

Sample should be coated with the resist promptly after surface preparation. If this is not possible, good adhesion can still be obtained after a prolonged period of storage if the sample has been protected from surface hydration or other contamination. The AZ 5214-E

photoresist is selected for the UV lithography. It offers exceptionally straight sidewalls and 
high thermal stability (up to $\sim 150{ }^{\circ} \mathrm{C}$ ) to maintain accurate pattern transfer during ion mill and reactive ion etching. In addition, the AZ 5200 Series resist can image reverse to a negative tone using a simple post-exposure bake (PEB), which adds extra versatility to the process. The last two digits of the numerical designation correlate to the viscosity of the photoresist, which, in the case of 5214, would give a coating thickness of $1.4 \mu \mathrm{m}$ at 4000 rpm spin for 40 seconds. A suitable amount of resist should be applied for optimal coating of the sample without inundating the edges and back surface with the resist. It is noted here that because the substrate size is a mere $1 \times 1 \mathrm{~cm}$ square, there is an aggregation of photoresist, called the edge bead, near the edges and at the corners of the sample due to surface tension, after the resist is spun on the substrate. Successful patterning of structures on the order of microns hinges on the proper removal of the edge bead.

\subsubsection{Softbake}

Softbaking removes most of the remaining solvent from the photoresist film, thereby densifying it. The softbake time and temperature can influence adhesion, photospeed, and dimensional control of the photoresist. Also, they affect the selectivity of the developer between exposed and unexposed regions of the photoresist. Monitoring the amount of unexposed resist loss during development provides an effective control parameter for process

optimization, given that the unexposed resist loss during development changes inversely to softbake time and temperature. A softbake temperature of $95{ }^{\circ} \mathrm{C}$ for only 45 seconds on a hot plate is applied to the sample to optimize the sensitivity and contrast of the photoresist. The short bake time does not appear to compromise the adhesion and stability of the resist. 


\subsubsection{Photomask and Exposure}

The photomask use to exposed the pattern is first virtually designed in the software L-Edit by Tanner Research. Other software like the more popular AutoCad can also be used. The basic idea in the mask design is to separately write the pattern for each layer into its own die. The patterning for the heterostructure injection devices includes four major stages, i.e., the CMR mesa, YBCO bridges, Au contacts, and contact wires, so four die patterns are needed in the design. Although within the design program, the layers could be viewed as overlapping patterns, it is important to add ample number of position markers through the die to allow for swift and accurate alignment of each pattern relative to its precursor. For edge bead removal, an additional die with a simple opaque square pattern nominally smaller than the sample should be included on the mask design.

The five die patterns are written to the same photomask $(4 \times 4$ in. $)$, made of either quartz or white crown glass, with the former being the most expensive option. On one side of the glass is a layer of chrome, on which the die patterns are written. The chrome is covered with an anti-reflective coating. The patterns are written on the photomask either by e-beam or photolithography. E-beam lithography is used to make the photomask, because the smallest feature requirement in this design is $\sim 1 \mu \mathrm{m}$.

The photosensitivity of the AZ 5214-E photoresist in positive tone is suitable for the $313,334,365$, and $405 \mathrm{~nm}$ emission peaks of the standard mercury vapor light source. These wavelengths can be used to achieve resolution of one micron and below in the $1.4 \mu \mathrm{m}$ thick resist films. It is important to achieve close contact between the mask pattern and the sample covered with the photoresist film; in general, soft contact mode, which involves 
bringing the sample and the mask to light mechanical contact, can be used for large features in the patterning. For structures on the order of a few microns, it is necessary to activate the hard contact vacuum mode. Here a vacuum gasket seals against the photomask the volume near the sample, and air is evacuated via a small pump, pressing the sample flush against the photomask to ensure very tight contact. When using this mode, it is important to use the edge bead removal die to overexpose and then develop out the edges of the photoresist, because these large edge bead, if not removed, would physically impede close contact between the sample and the photomask. Depending on the intensity of the mercury lamp, typical exposure times for AZ 5214-E resist range between 12 to 36 seconds; however, for edge bead removal, exposure of several minutes can be used.

\subsubsection{Development and Rinse}

The developer used is an odorless aqueous AZ inorganic sodium-based developer. It is buffered to maintain a uniform $\mathrm{pH}$ and to provide maximum bath life and process stability. The dilution used is 1 part developer to 1 part deionized (DI) water. This dilute concentration enables higher contrast and provides greater selectivity between the exposed and unexposed resist. A longer development time of approximately 1 minute may be necessary. The developer should be maintained at a constant temperature $\left( \pm 1{ }^{\circ} \mathrm{C}\right)$ within the range of $20-25{ }^{\circ} \mathrm{C}$. The sample upon completion of development should be rinsed immediately with DI water to halt the developing process. To dry the DI water to avoid prolonged exposure of YBCO to water, the sample can be spin dried or forced dry with filtered nitrogen. 


\subsubsection{Postbake and Photoresist Removal}

Postbaking, also called hard baking, will generally improve image stability and adhesion as well as plasma and chemical resistance. The postbaking parameter can vary widely, but for this application, baking on a hot plate at $105{ }^{\circ} \mathrm{C}$ for 20 minutes is recommended. After the etch process is complete, the photoresist generally can be removed by soaking in acetone first for 10 minutes and then in ultrasound for an additional 5 minutes. Isopropanol and ethanol solvents typically follow to rinse off the remnant residue.

\subsection{Device Microfabrication}

The samples used to make these patterned devices are again fabricated by Dr. R. P. Vasquez at the Jet Propulsion Laboratory. These samples are similar to the ones made using the shadow masking, described in Sec. 3.1, except that the samples are larger squares $(1 \times 1 \mathrm{~cm})$ and that an additional layer of gold has been pre-evaporated in situ, allowing for contacts to be made to the devices. This additional Au layer also serves the purpose of a protective layer to the YBCO film underneath, as the procedures of lithography require sample to come in contact with water and other organic solvents, which may prove to be detrimental to the survival of the superconductor otherwise. The first set of F-I-S devices is made on YBCO/STO/LSMO of thicknesses $100 \mathrm{~nm} / 2 \mathrm{~nm} / 100 \mathrm{~nm}$, and the second set

on similar sample of thicknesses $100 \mathrm{~nm} / 3.5 \mathrm{~nm} / 150 \mathrm{~nm}$. Partner samples with LNO underlayer are processed as well as controls.

The procedures for the microfabrication can be divided into four major stages to achieve these features: CMR mesa, YBCO bridge, Au contacts, and contact wires. Each 


\section{Stage 1: CMR Mesa}

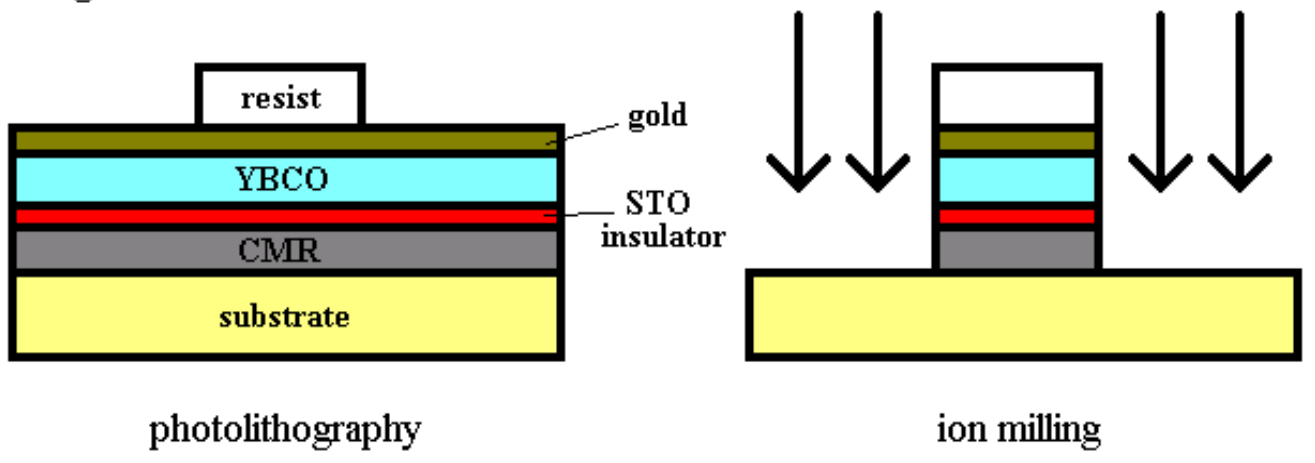

Figure 8.1: First stage in the microfabrication to obtain the large CMR mesa.

stage includes similar series of steps. The details on photolithography (Sec. 8.1) and thin film processes (Chapter 7) will be abbreviated to avoid repetition. Relevant comments will be noted.

First, the photolithography procedures discussed in the previous section are followed to obtain the patterns for the large CMR mesa structures. The sample is then placed in the ion etch chamber for a quick reactive $\mathrm{O}_{2}$ ion etch at low power (20\%) in 40 mTorr for 20 seconds. This serves to remove any residual layer of organic material in the exposed region in the patterning. To etch the CMR mesa, an ion beam at $500 \mathrm{~V}, 50 \mathrm{~mA}$, with an accelerator voltage at $800 \mathrm{~V}$ is applied. The milling pressure is $\sim 2 \times 10^{-4}$ Torr. The ion milling rate for the $\mathrm{YBCO}$ and $\mathrm{CMR}$ layers are comparable, with the CMR layer etching nearly $10 \%$ slower. After the CMR mesa etching is complete, the photoresist is stripped with acetone, etc., and another reactive $\mathrm{O}_{2}$ ion etch for 5 minutes follows to remove the deposits and other adhesions on the sample surface from the photoresist and the organic solvents used for resist removal. To confirm the mesa height, a profilometer is used to 


\section{Stage 2: YBCO Bridge}
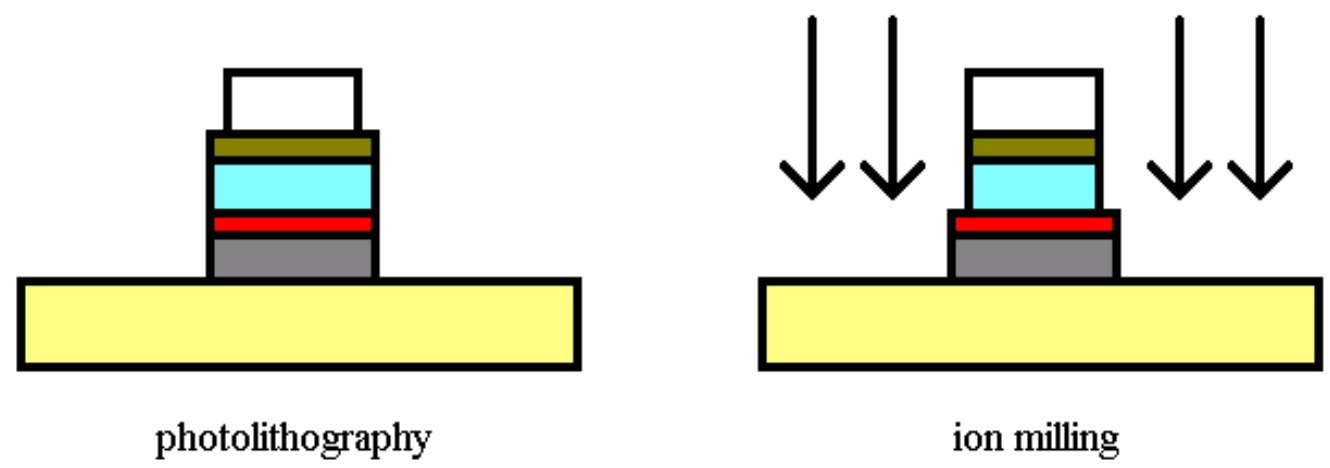

Figure 8.2: Second stage in the microfabrication to obtain the YBCO bridges.

measure an average height across the edges of the mesa.

The second stage involves patterning the narrow YBCO bridges. Here the procedure mimics those described for the CMR mesa. However, the effect of edge beads become a factor, so their removal need to be addressed. It is also necessary to align the patterns properly relative to the existing CMR mesa. As one may expect, since the etch depth should extend only down to the CMR layer (as opposed to all the way to the LAO substrate), the etch time should be reduced, according to the calibrated rate. Again, the bridge height should be verified using the profilometer.

The third stage of the fabrication process is to obtain the Au contacts. The photolithography requires a rather difficult alignment procedure as some of the contacts have dimensions of only a few microns. Successful patterns for contacts of these sizes require very clean sample surface and good photomask-to-sample contact. Since the material to be etched is the top Au layer of the sample, which has a much faster etch rate compared to perovskite materials, the ion beam voltage and current are decreased to $100 \mathrm{~V}$ and 10 


\section{Stage 3: Gold Contacts}

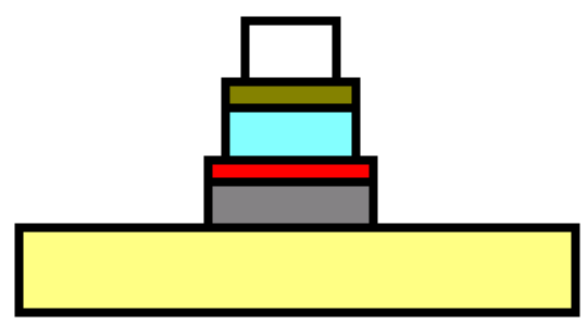

photolithography

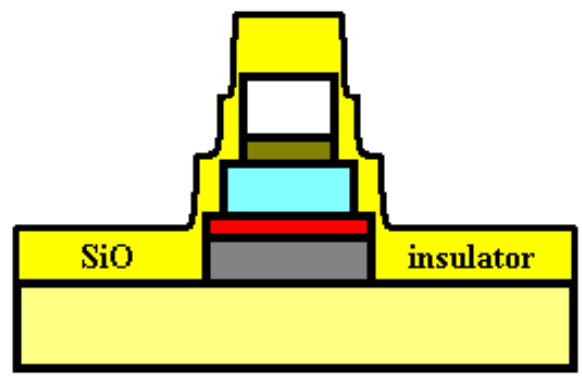

insulator deposition

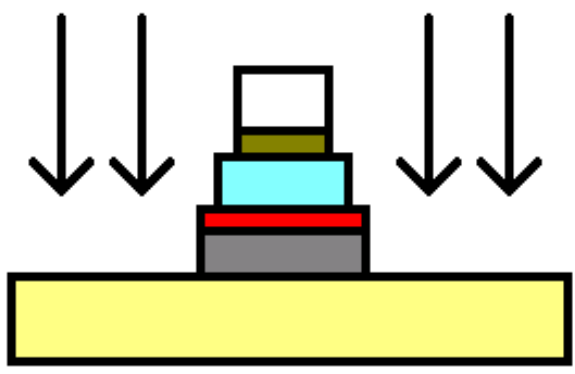

ion milling

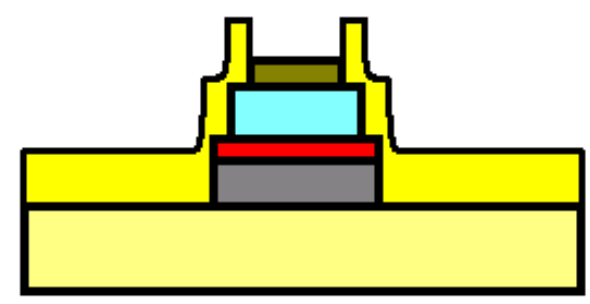

resist lift-off

Figure 8.3: Third stage in the microfabrication to obtain the Au contact pads and to add insulator to prevent shorting in the devices.

$\mathrm{mA}$, respectively. Immediately after the ion etch, a layer of $\mathrm{SiO}$ or $\mathrm{SiO}_{2}$ insulator layer of thickness $200-300 \mathrm{~nm}$ is deposited on the entire substrate surface. Therefore, upon removal of the photoresist after the deposition step, only the previously protected Au contact areas will be exposed for the purpose of electrical contact, and everywhere else will be covered by this insulator layer.

The last stage is the patterning of the gold contact wires from the edge of the sample to the Au contacts on the devices. The lithography performed in this step can be the same process as in previous stages, or the negative-tone resist can be used, where the 


\section{Stage 4: Contact Wires}

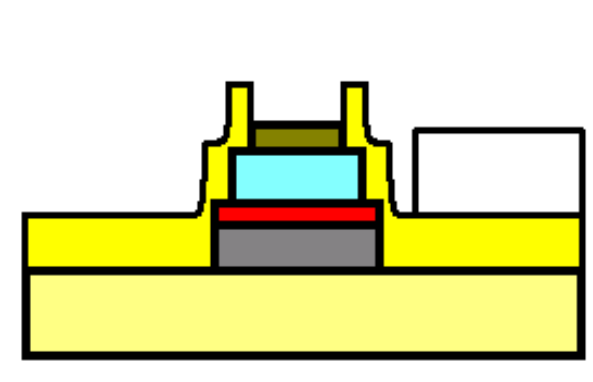

photolithography

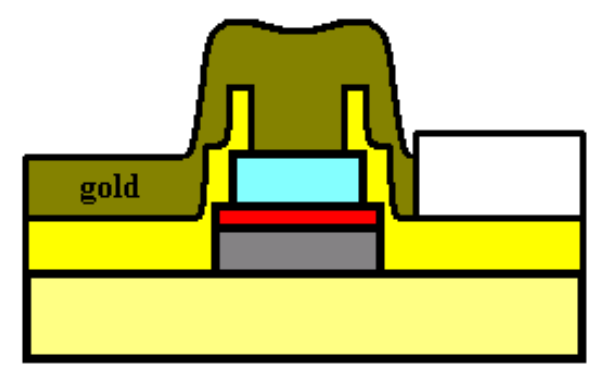

ion milling

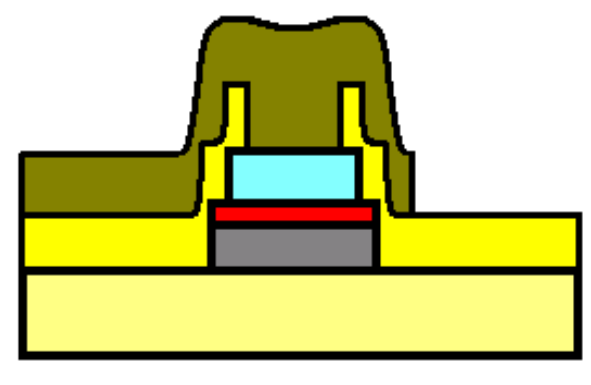

\section{Completed}

Device

\section{lift-off}

Figure 8.4: Final stage in the microfabrication to obtain the contact wires.

image reversal is accomplished via a simple post-exposure bake process. The deposition of gold is done in an evaporator for about $3-4$ minutes for a thickness of $\sim 200 \mathrm{~nm}$. To assist in the adhesion of $\mathrm{Au}$ to the $\mathrm{SiO} / \mathrm{SiO}_{2}$ insulator layer, a $5 \mathrm{~nm}$ primer layer of $\mathrm{Ti}$ is deposited prior to the Au deposition. The two metal layer deposition can usually be evaporated in the same evaporation chamber in one single pump down. Then the photoresist removal and reactive $\mathrm{O}_{2}$ ion etch cleaning completes this last stage of processing. Fig. 8.5 is a photographic image taken of the sample on the scale of nearly the entire die, with the devices located near the center of the square die. Fig. 8.6 shows the close-up of the F-I-S devices. 


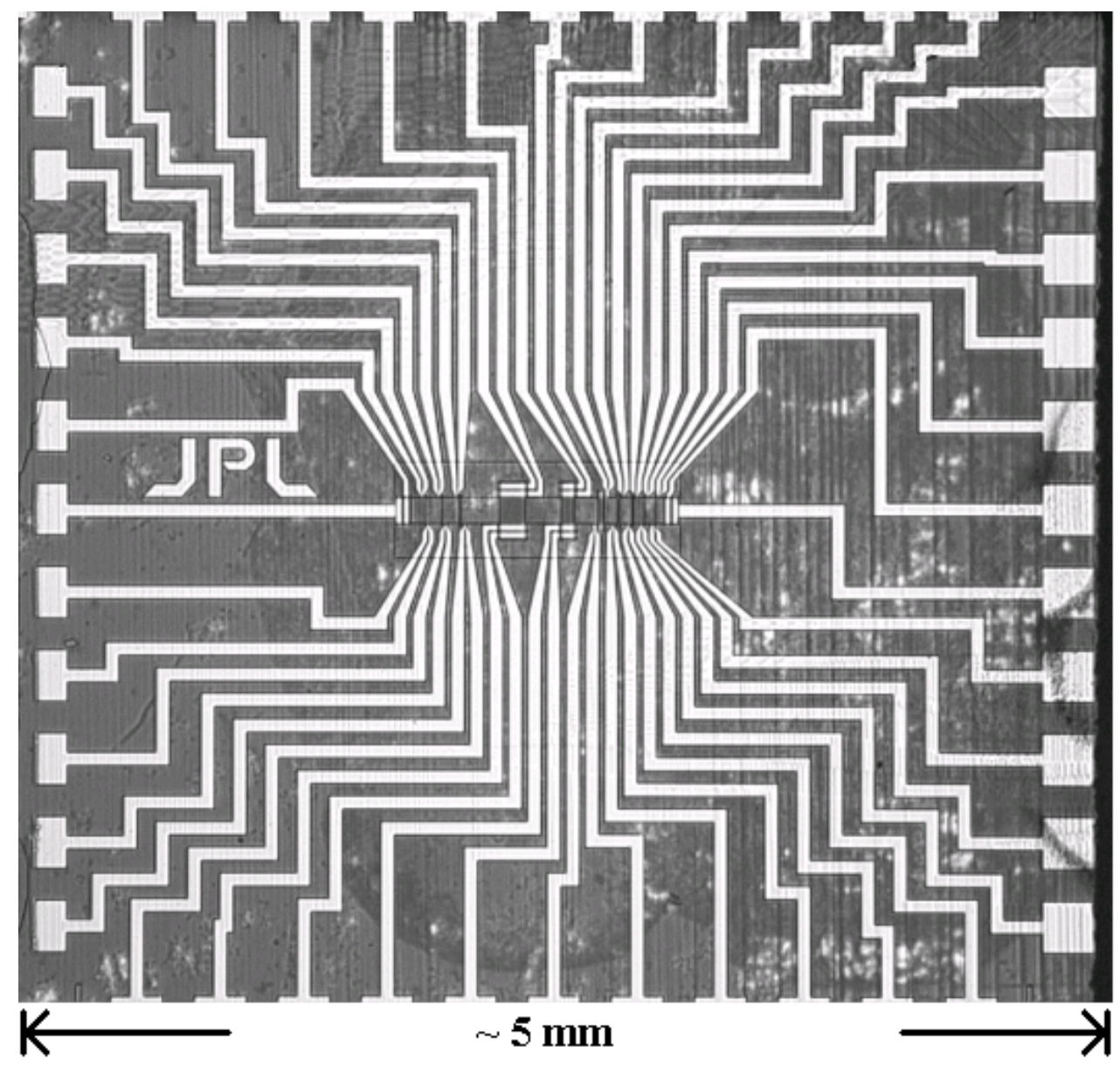

Figure 8.5: Image of one spin injection sample, which was fabricated at JPL. The mask used for patterning of this sample was designed by Dr. Jeffrey Barner and Dr. Alan Kleinsasser. 


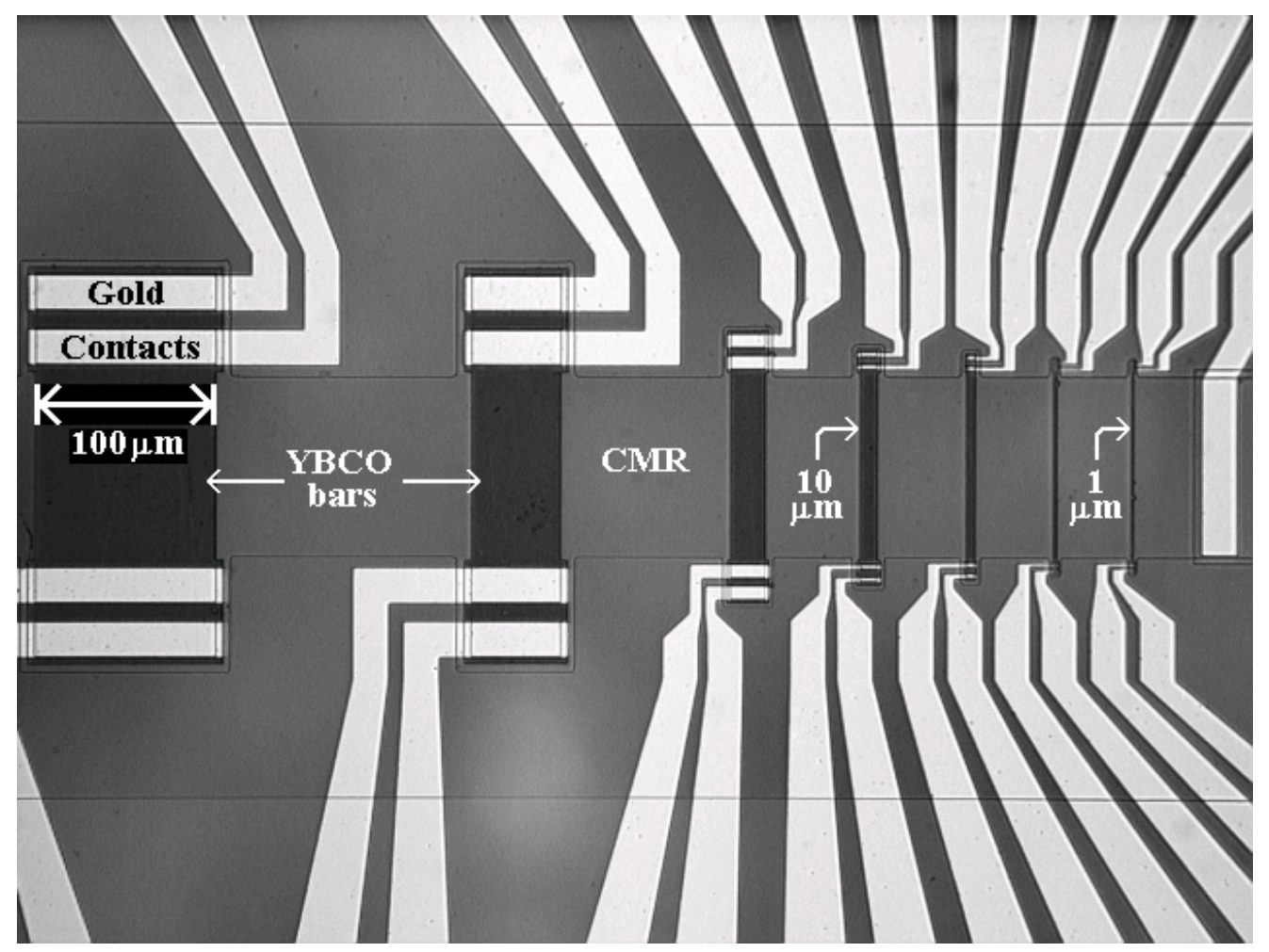

Figure 8.6: Close-up view of the F-I-S devices. 


\subsection{Technique}

The pulse-current technique was employed in the as-grown samples to eliminate Joule heating resulting from passing high currents through the resistive CMR underlayer. In these patterned samples, however, since the dimensions of the YBCO bridges can be miniaturized, the magnitude of the injection current required to accomplish similar level of current density is significantly reduced as a result. In addition, the length of CMR material, and thus the total resistance that the injection current is required to pass through before injecting into the superconductor, can be shortened by the patterning of devices. The net benefit of these factors is that the simpler technique of using dc currents can be used for injection measurements in these patterned devices because of the much reduced Joule heating. To determine the range of applied dc currents that yield negligible heating effects, I-V curves on the CMR layer are performed and the characteristics are found to be Ohmic for currents up to roughly $\sim 15-20 \mathrm{~mA} / \mu \mathrm{m}$. So for a CMR width of $100 \mu \mathrm{m}$, heating effects can be considered negligible for currents below $15 \mathrm{~mA}$. Another notable advantage with using dc current sources is that the current paths of the supercurrent and injection current are well defined, because the two current sources have large output impedances and do not need to synchronize and thus share a common ground.

The basic measurement of critical current of the superconductor is identical to that of a 4-contact point measurement discussed in Sec. 4.5. However, to determine the critical current, the full I-V characteristic curve needs to be mapped out. For the injection current, a second current source is connected to the injection device, with positive electrode on a CMR contact and the negative electrode on the YBCO contact. This is done to mimic 
the current flow in the pulse-current technique.

\subsection{Interface Resistance and Transfer Length}

The contact resistances of the interface between the superconducting and CMR layers in the as-grown heterostructures cannot be measured directly, although the values have been estimated on the order of a few $\mu \Omega$. This is due to the fact that the contact resistance can only be measured in series with the resistances of the YBCO and CMR material. Given that the dimensions of these layers are not known accurately in the asgrown heterostructures, an exact analysis of their values proves to be difficult.

In the patterned injection devices, on the other hand, the interface resistance can be obtained because of the precise control of the sample dimensions available through lithographic patterning. To determine the interface resistance, a transmission line model between materials of high and low resistances can be applied $[132,133,125]$. This model assumes that as current $I$ flows in the resistive CMR layer, a fraction of the current $d I$ will tunnel across the insulator into the superconductor in the distance of $d x$ along the interface. The voltage drop between the CMR and the grounded superconductor layers is given by

$$
V(x)=\frac{\rho_{c}}{w} \frac{d I(x)}{d x}
$$

where $\rho_{c}$ is the specific interface contact resistance, and $w$ is the width of the contact interface of the two layers. On the other hand, the remaining current continues along the CMR layer and will also suffer a voltage drop $d V$ in a distance $d x$ :

$$
\frac{d V(x)}{d x}=\frac{R_{s}}{w} I(x)
$$




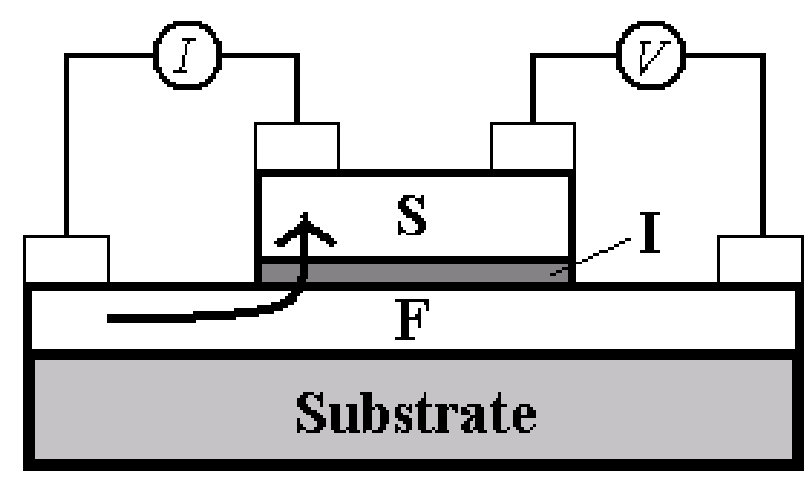

Figure 8.7: Schematic for transfer length measurement.

where $R_{s}$ is the sheet resistance ( $\Omega$ /square) of the CMR layer. Combining the two equations gives the differential equation

$$
\frac{d^{2} V(x)}{d x^{2}}=\frac{1}{d_{T}^{2}} V(x),
$$

with

$$
d_{T}=\sqrt{\frac{\rho_{c}}{R_{s}}}
$$

being the current transfer length. By injecting a current into the superconductor from one side from the CMR layer and monitoring the voltage between the two layers from the other side, as shown in Fig. 8.7, one can empirically determine the characteristic parameters using

$$
\frac{V(d)}{I}=\frac{\sqrt{R_{s} \rho_{c}}}{w} \operatorname{csch}\left(\frac{d}{d_{T}}\right) .
$$

Using the above model, the measurement is performed on the $10 \times 100 \mu \mathrm{m}^{2}$ device with $2 \mathrm{~nm}$ STO barrier at LHe temperature. The resistances $R_{s}$ and $V(d) / I$ are determined to be $\sim 44 \Omega$ and $6.3 \mathrm{~m} \Omega$, respectively. Given that $d=10 \mu \mathrm{m}$ and $w=100 \mu \mathrm{m}$, the value 
obtained for $\rho_{c}$ is $1.4 \mu \Omega-\mathrm{cm}^{2}$. Consequently, the transfer length for the injection current to flow into the superconductor from the CMR film is $d_{T}=1.8 \mu \mathrm{m}$. This analysis for the $2 \mathrm{~nm}$ STO sample came from data measured and results derived at JPL (A. Kleinsasser et. al., private communication). For the sample with $3.5 \mathrm{~nm}$ STO insulator, a transfer length of $\sim 74 \mu \mathrm{m}$ is derived. The much longer transfer length for the thicker barrier sample is not surprising. A deposited barrier of $2 \mathrm{~nm}$ is not expected to be continuous, i.e., pin holes could compromise the integrity of the barrier. The $3.5 \mathrm{~nm}$ insulator should provide a more uniform barrier, and as a result, the distance of current transfer is significantly lengthened.

\subsection{Results}

As described earlier in this chapter, these devices undergo many cycles of processing that include hot plate heating, ion etching, cleaning by organic solvents, etc. To verify the quality of the devices, resistivities of the LSMO/LNO and YBCO layers are measured. For the CMR layer, data is taken during sample cool down and are plotted as a function of temperature in Fig. 8.8. The $R$-vs.- $T$ curves show monotonic behavior up to room temperatures for both LSMO and LNO. This means from the previous CMR film characterizations that the $T_{\text {Curie }}$ should still be near the value of $320{ }^{\circ} \mathrm{C}$ and that the quality of the film remains high. Also, the overall magnitude of the resistivity is comparable to that of the as-grown samples.

The YBCO bridges, grown on LSMO film, consist of lines of width 100, 50, 20, 10, 5, 2, and $1 \mu \mathrm{m}$. Their $\rho$-vs.-T curves are shown in Fig. 8.9. The normal-state YBCO bridges exhibited larger resistivity, by a factor of 2 to 3 , than those of single crystals and 


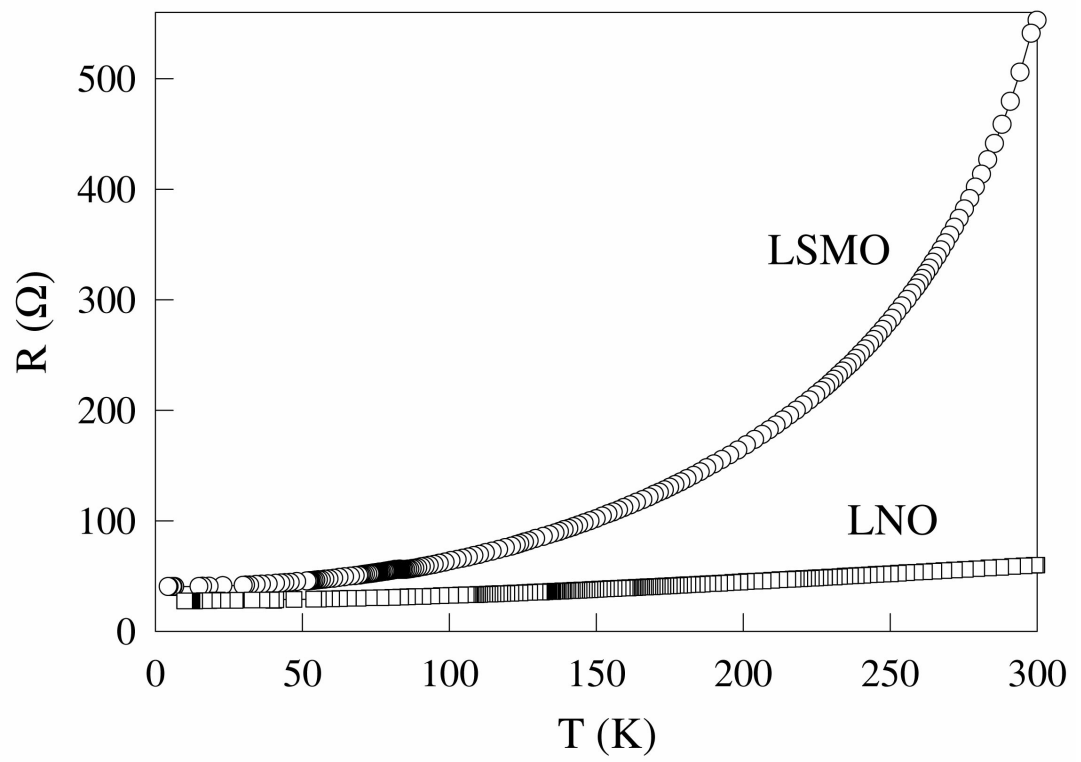

Figure 8.8: $R$-vs.- $T$ curves for CMR materials.

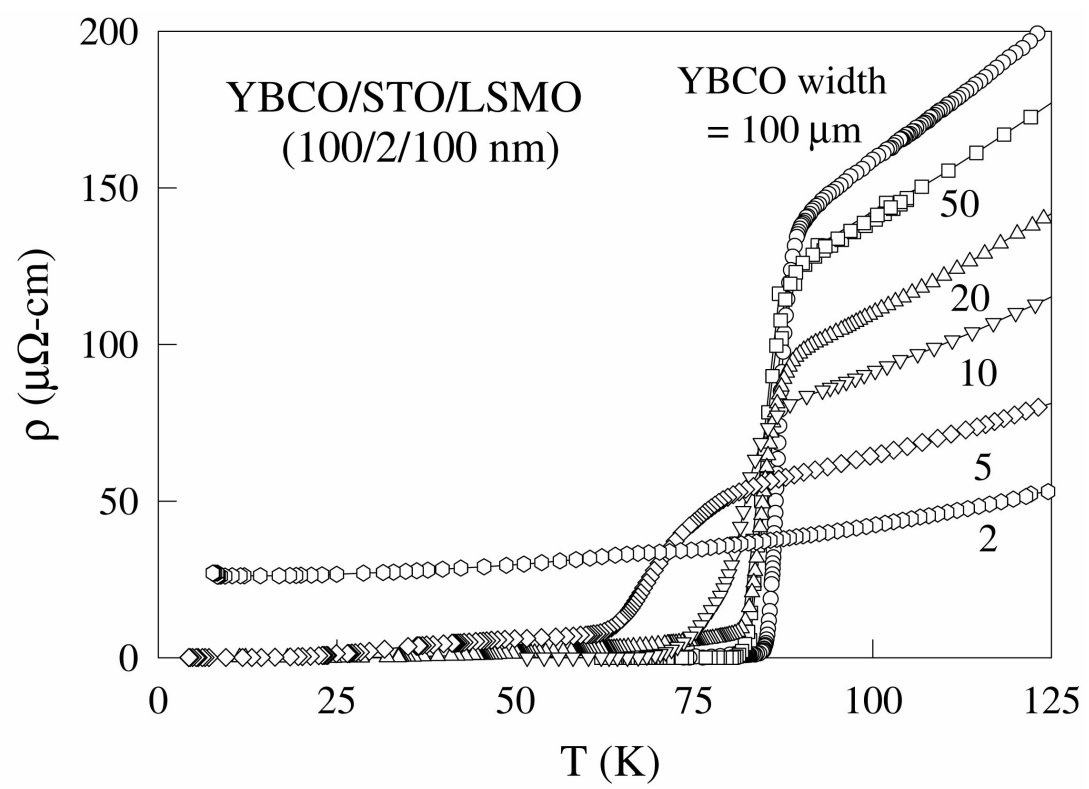

Figure 8.9: $\rho$-vs.- $T$ curves for YBCO in the F-I-S devices of various bridge widths. $T_{c}$ appears to deteriorate as the width decreases. The narrow $2 \mu \mathrm{m}$ bridge does not undergo the superconducting transition. 


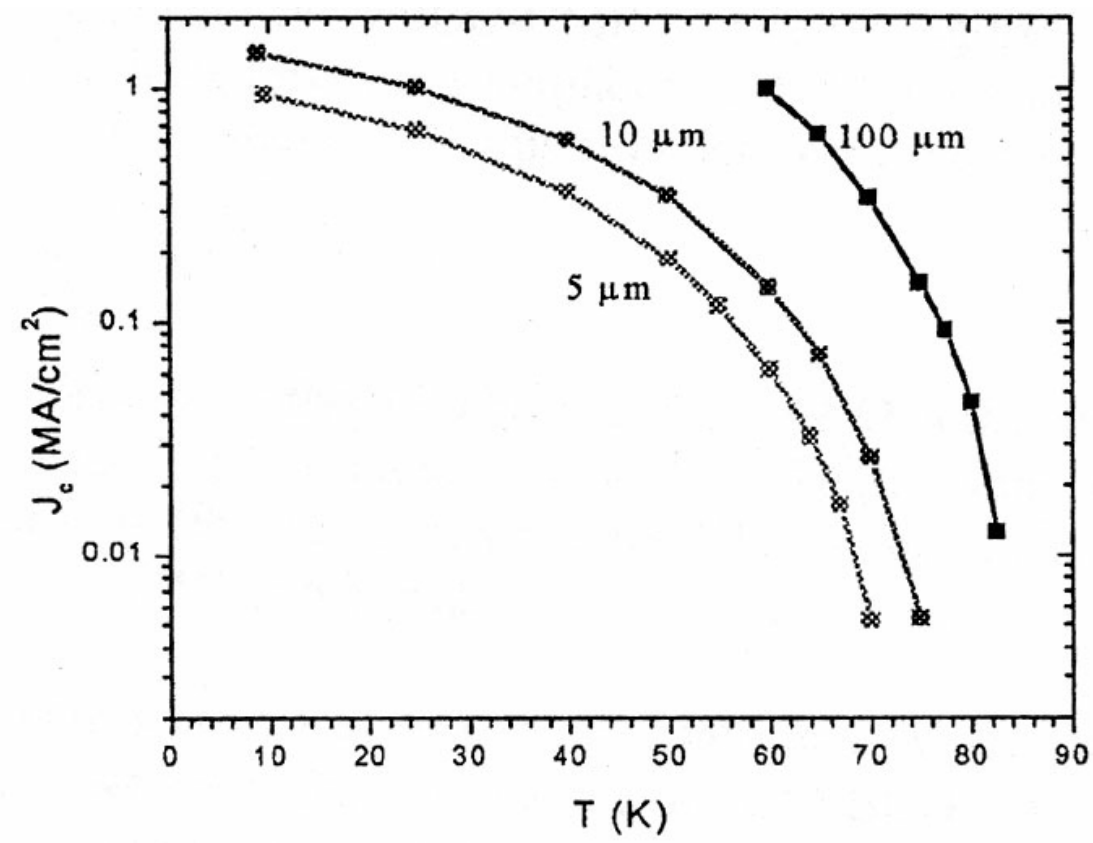

Figure 8.10: $J_{c^{-v s}}$ - $T$ plot for $5,10,100 \mu \mathrm{m}$ wide YBCO devices.

as-grown heterostructures, suggesting some deterioration of the bulk sample quality after device processing. In general, the superconducting transitions occur below $90 \mathrm{~K}$, with the wider bridges having higher $T_{c}$ 's. The superconducting transition widths vary from as small as $\sim 5 \mathrm{~K}$ in the $100 \mu \mathrm{m}$ bridge to $\sim 10 \mathrm{~K}$ in the $5 \mu \mathrm{m}$ bridge. Although the 2 and $1 \mu \mathrm{m}$ bridges remain electronically continuous, they are no longer superconducting after undergoing the process of lithographic patterning. It is likely that the edge effect from ion damage that may have destroyed the stoichiometry of the YBCO superconductor during the etch process is on the order of a few microns. In the bridges of widths 100, 10, and $5 \mu \mathrm{m}$, the critical currents $J_{c}$ are obtained from I-V curve scans, as shown in Fig. 8.10. Here the critical current values are largest for the $100 \mu \mathrm{m}$ bridge. Spin injection experiments performed on these patterned F-I-S devices show only small $J_{c}$ suppression, in 


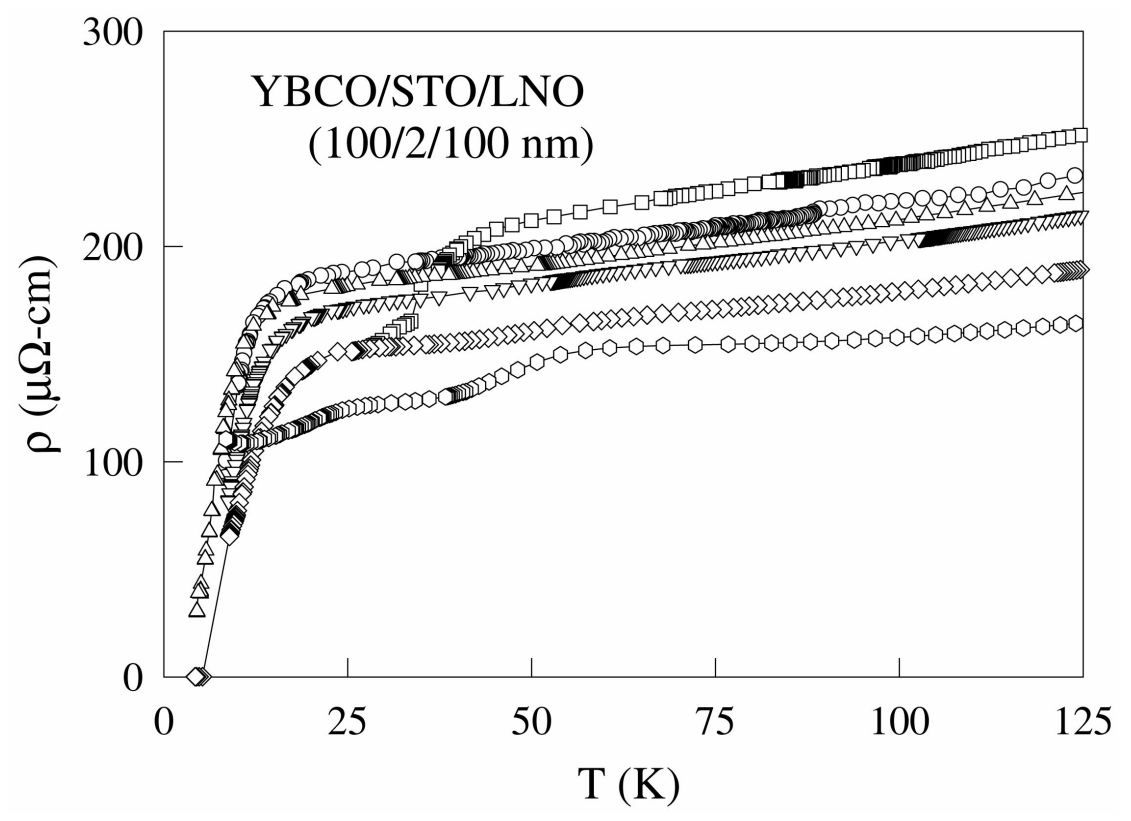

Figure 8.11: $\rho$-vs.- $T$ curves for YBCO in the N-I-S devices of various bridge widths. $T_{c}$ appears to deteriorate significantly after processing, as $T_{c}$ dipped below $20 \mathrm{~K}$.

general. However, since the current transfer length of $1.8 \mu \mathrm{m}$ for STO thickness of $2 \mathrm{~nm}$ coincides loosely with the width of ion damage region, the degree of spin polarization for the injected currents in the patterned F-I-S devices might have been weakened considerably because of strong magnetic impurity scattering within the damaged region at the interface. Hence, it is difficult to draw reliable conclusions from data taken on these devices with a very short transfer length.

A control sample with the same YBCO bridges grown on LNO is simultaneously processed. Some YBCO lines appear to have very low $T_{c}$ 's $(<20 \mathrm{~K})$, and others have not displayed superconductivity down to the lowest temperature measured (see Fig. 8.11). Evidently, interdiffusion of atoms and other reactions, which are facilitated because the extreme conditions during processing, have taken place between the YBCO and LNO layers. 


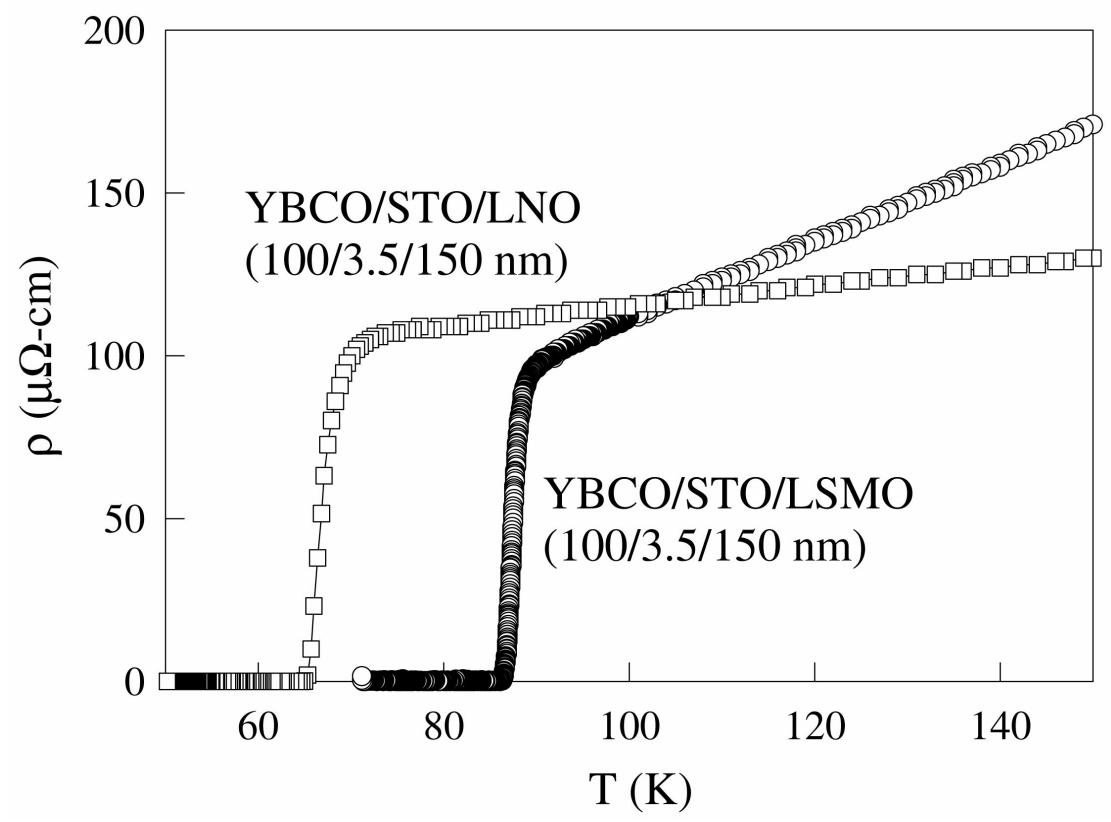

Figure 8.12: $\rho$-vs.-T curves for 100- $\mu \mathrm{m}$ width YBCO bridge in both F-I-S and N-I-S devices. $T_{c}$ appears to improve as the thickness of the insulating STO increases.

To improve the probability of good quality control devices, two LSMO and LNO partner samples with a $3.5 \mathrm{~nm}$ STO buffer layer are processed. In Fig. 8.12, the $\rho(T)$ data for the pair of $100 \mu \mathrm{m}$ YBCO bridges show that with the thicker insulating layer, the quality of the devices has been dramatically enhanced, with $T_{c}$ above $75 \mathrm{~K}$ in both samples with LSMO and LNO control underlayers. With both samples showing good superconducting characteristics, the dc injection currents are then applied. By monitoring the $I-V$ curves, the values of $I_{c}^{+}$and $I_{c}^{-}$are extracted and analyzed. In Fig. 8.13(a) and (b), the normalized $J_{c}$ versus $I_{i n j}$ curves at various reduced temperatures for these $100 \mu \mathrm{m}$ bridges are shown. In the device with LSMO injection underlayer, a progression of increased suppression of $J_{c}$ is observed as $T \rightarrow T_{c}$. In contrast, at similar reduced temperatures, the LNO underlayer sample shows markedly smaller effect from current injection. This again can be attributed 

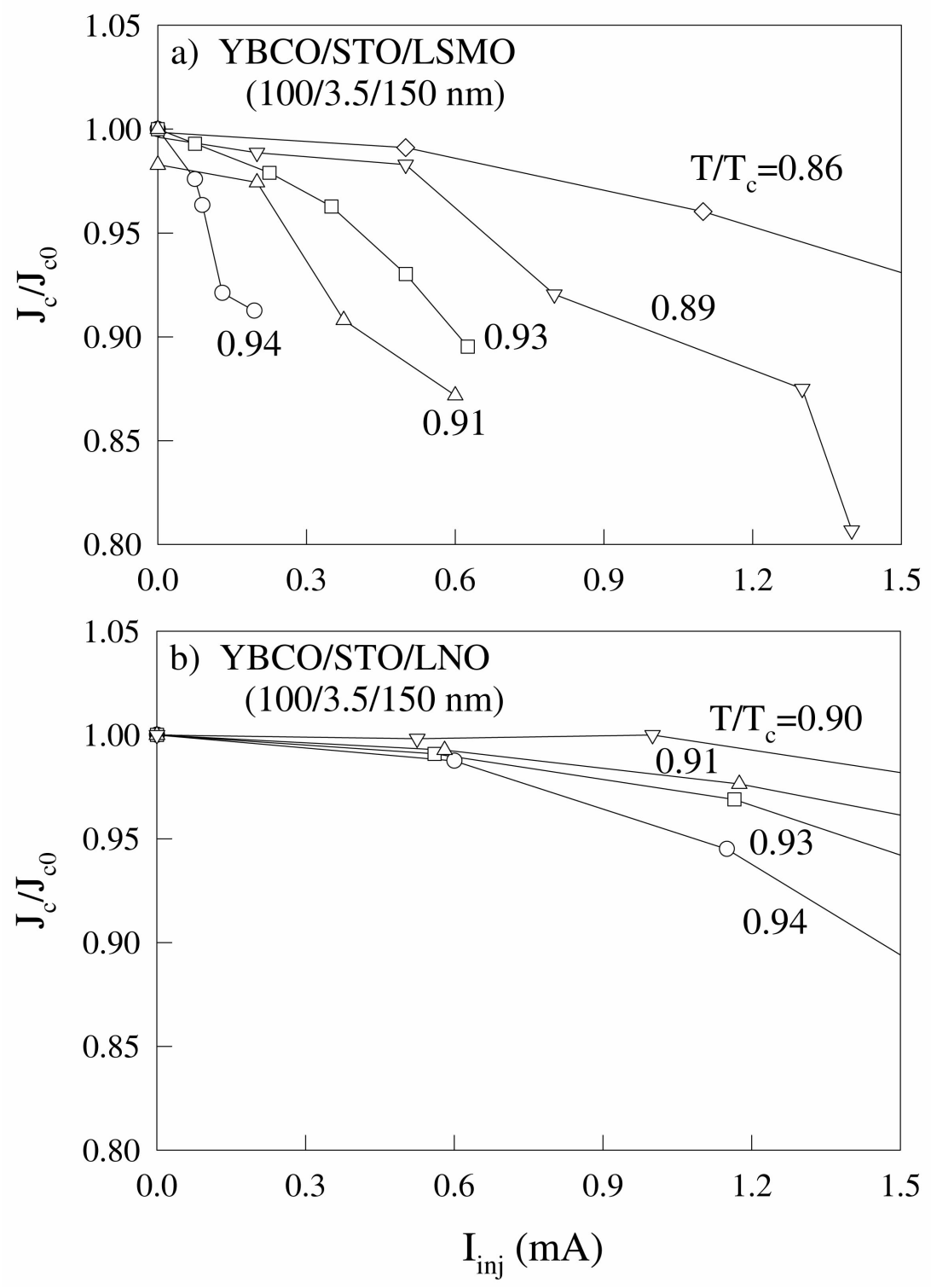

Figure 8.13: Normalized $J_{c}$ vs. $I_{i n j}$ for YBCO on a) LSMO, b) LNO. Both devices have width of $100 \mu \mathrm{m}$. 

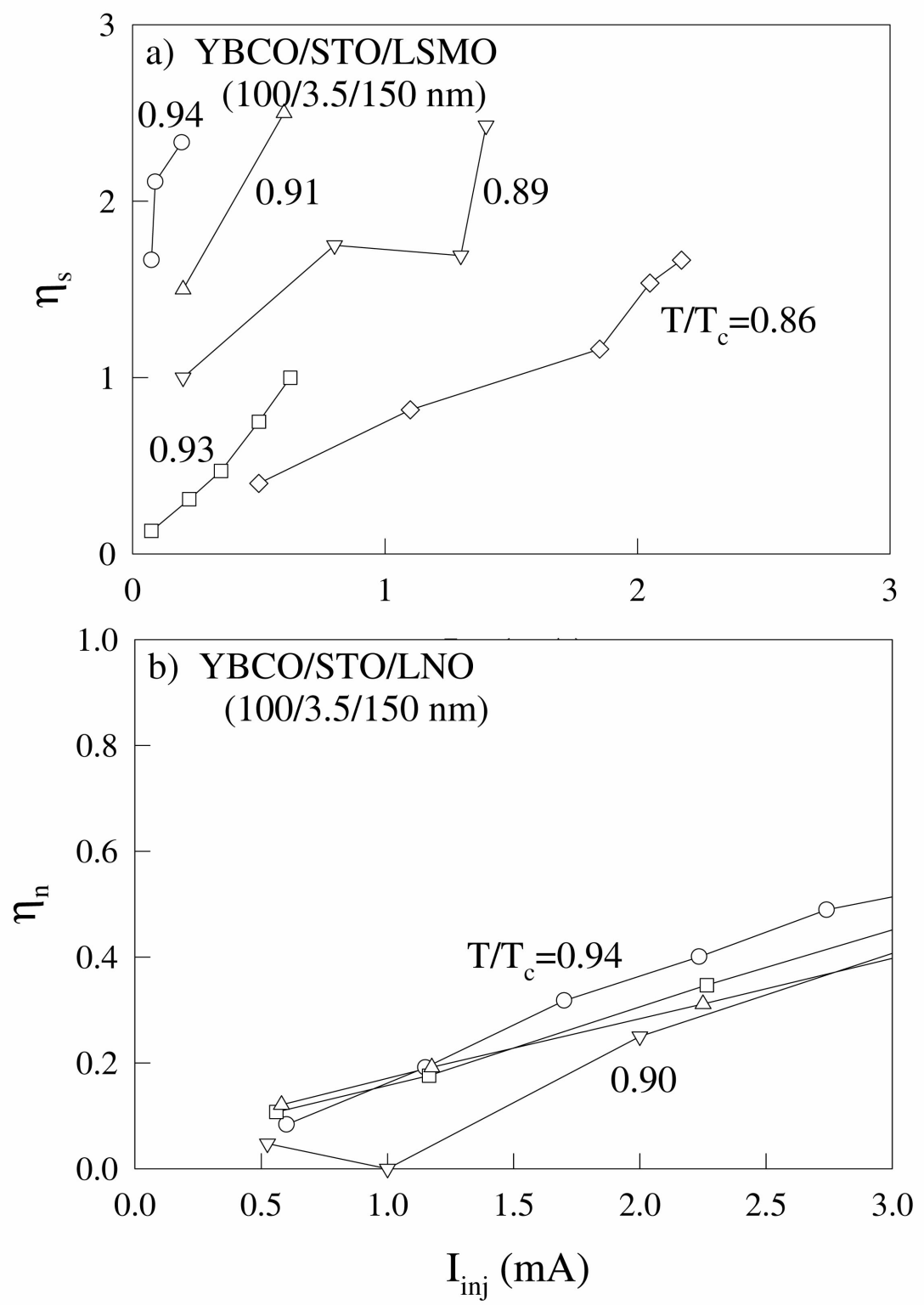

Figure 8.14: a) $\eta_{s}$-vs.- $I_{i n j}$ data for F-I-S; b) $\eta_{n}$-vs.- $I_{i n j}$ data for N-I-S. 
to the injection of spin-polarized quasiparticles into the YBCO superconductor from the LSMO film. The normal quasiparticles injected from the LNO film have a much shorter lifetime after entering the superconductor and quickly recombine into pairs, so their effect is significantly diminished as a result.

With further analysis, the efficiency exhibits a monotonic increasing dependence on the injection current density in both devices, as shown in Fig. 8.14(a) and (b). However, the magnitude of efficiency in the spin-polarized quasiparticle injection case is elevated, with some values exceeding unity. The large efficiency values, which sharply drop off in the low injection level regime observed in the as-grown samples, is absent here, as these measurements are not able to be carried out at low temperatures because the critical currents are too high. Often, the miniaturized contact wires are not able to handle current loads on the order of a few tens of milli-amperes.

\subsection{Summary}

Spin-injection microdevices and their control counterparts have been successfully fabricated using lithographic patterning and etch techniques. It is found that for higher probability that the YBCO superconductor film's survival through device processing, a thicker insulating buffer is required in the growth of the heterostructures. By patterning the devices to precise dimensions, the contact resistance and transfer lengths of the injection current are determined for the interfaces in these heterostructure devices. In the pair of good quality spin-injection and control devices, a large contrast in the effect of current injection is observed, where a more significant suppression of $J_{c}$ and higher efficiency levels 


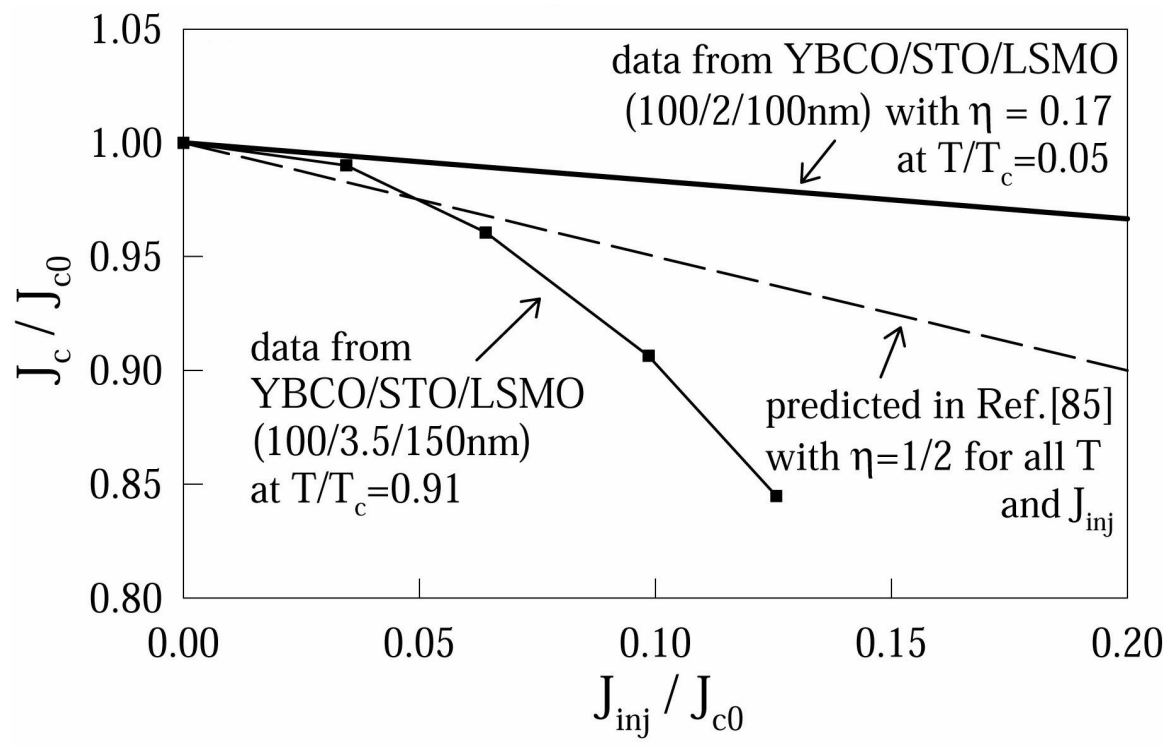

Figure 8.15: Normalized $J_{c}$ data from two patterned devices are compared with the hypothetical gain of $1 / 2$, predicted by the geometrical effect of current summation [116].

are observed in the LSMO spin-injection device. These results therefore confirm the strong effect of spin-polarized quasiparticles on cuprate superconductors and are qualitatively consistent with our findings in as-grown heterostructures.

A comment should also be made in the context of these devices regarding current summation. It is proposed in such a scenario that a minimum efficiency of $1 / 2$ should be observed for injection experiments, which supposedly arises from a geometric effect [125]. In Fig. 8.15, the normalized $J_{c}$ is plotted versus $J_{i n j}$, normalized by $J_{c 0}$. Here the dashed line represents the requisite minimum efficiency that should be present in all measurements. Again the device with the $3.5 \mathrm{~nm}$ STO insulator exhibits a more pronounced suppression of $J_{c}$. However, the contradiction to the current summation can clearly be seen in the data from the $2 \mathrm{~nm}$ buffer device, where an efficiency value of $\sim 0.17$ is measured. Such a result would be impossible should an efficiency baseline of $1 / 2$ exist. Thus, the data from these 
patterned devices, in addition to those from the as-grown heterostructures, also refute the validity of current summation. 


\section{Chapter 9}

\section{Conclusion}

In this final chapter, a brief summary of the study on spin-injection is presented. Other possible related experiments for future investigation are also discussed.

\subsection{Summary}

Systematic studies of the critical current density $\left(J_{c}\right)$ in perovskite F-I-S and N-I-S heterostructure with different thicknesses of the superconducting layer have been investigated. Many difficulties that arise from film material and substrate selection as well as technical issues involving the measurement probes and sample heating have been overcome. With the application of CMR perovskites in the heterostructures, their transport and magnetic properties as well as substrate-induced effects have been studied in detail. It is found that to obtain high-quality half-metallic CMR films, it is important to have lattice-matched epitaxial growth of the CMR films. Furthermore, it has been demonstrated that sharp contrasts in the temperature and injection current dependences exist between F-I-S and of 
N-I-S devices. The strong suppression of superconductivity in F-I-S due to current injection cannot be explained by either the paramagnetic effect or a simple current-summation effect. Phenomenological analyses of our data suggest that the strong influence of spin-polarized quasiparticles on $J_{c}$ and on the quasiparticle density of states of F-I-S samples may be due to their suppression of the antiferromagnetic correlation in the $\mathrm{CuO}_{2}$ planes of the superconducting cuprate. By applying conventional theory of nonequilibrium superconductivity to model the results, the strong effects of spin-polarized quasiparticles are manifested by the long in-plane spin relaxation time and large shift in the chemical potential. In contrast, no discernible chemical potential shift can be found in the N-I-S samples using the same analysis. The strong effects of spin-polarized quasiparticles are probably unique to the cuprates and other superconductors that exhibit coexistence of antiferromagnetic correlation and superconductivity, and are reminiscent of the significant suppression of superconductivity due to non-magnetic impurities that induce local magnetic moments on the neighboring $\mathrm{Cu}$-sites in the $\mathrm{CuO}_{2}$-planes. In contrast to the in-plane spin relaxation mechanism via exchange interaction, the $c$-axis spin transport mechanism may be dominated by inelastic spin-orbit interaction. In conclusion, these results have demonstrated phenomena of nonequilibrium superconductivity in cuprate superconductors and the strong effects of spin injection. Moreover, the data in this work are consistent with quasiparticles rather than solitons as relevant low-energy excitations in cuprate superconductors. Further theoretical studies for the microscopic interaction of spin-polarized quasiparticles with the background antiferromagnetic correlation in the highly anisotropic $d$-wave cuprates will be necessary to provide better understanding of the results. 


\subsection{Possible Future Investigations}

The YBCO cuprate system has proved to be highly anisotropic in its transport properties. In order to more accurately probe the relaxation length- and time-scales along the $c$-axis and $a b$-plane directions, new films and junction designs might be helpful. One method that comes to mind is to invert the layers of the films in the heterostructure, as shown in Fig. 9.1(a), which can be controlled during the film growth process. In this geometry, since the superconductor region is equipotential, the injection of quasiparticles should occur uniformly throughout the junction interface, as either a bias voltage or current is applied between the CMR and YBCO films. This approach effectively removes the issue of the transfer lengths of the injection current and provides a better probe of $c$-axis parameters. However, there are technical issues that involve the growth of the CMR film on top of the YBCO layer instead of a typical perovskite substrate, which may be an interesting materials enterprise unto itself.

A second technique involves a more difficult microfabrication technique, that is, to create an $a b$-plane junction, as illustrated in Fig. 9.1(b), instead of the $c$-axis junctions that have so far been experimented with. To create this type of junction, it is necessary to introduce lithographic patterning during the growth of the heterostructures, in order to expose a component along the $a b$-plane in the junction. These types of devices have been widely made in low-temperature superconductors and other semiconductor applications, and has also been applied to high- $T_{c}$ systems for high-performance S-N-S junctions.

The other variation in this type of spin-injection experiment is to replace the YBCO superconductor, which so far has been the one constant in the investigation. An 
(a)

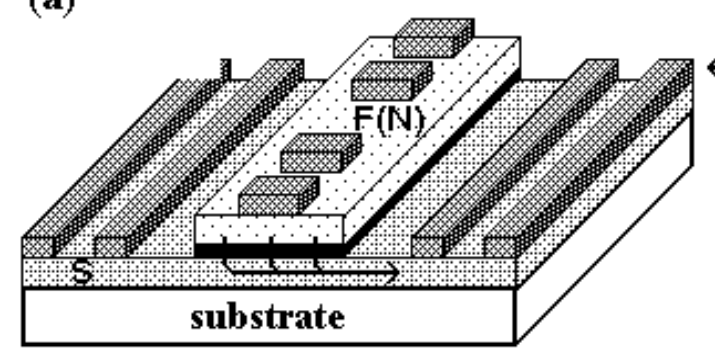

(b)

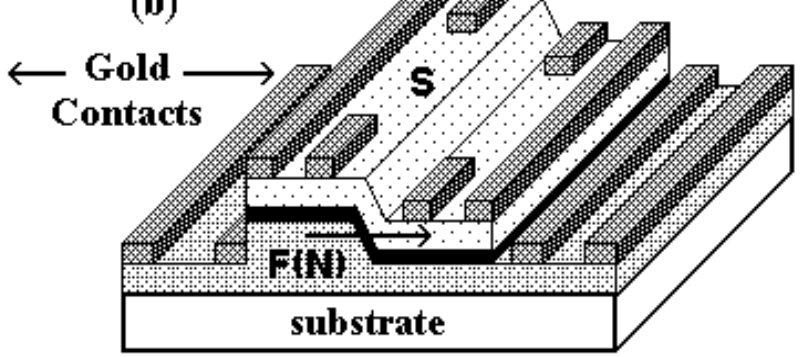

Figure 9.1: Other possible geometries for future heterostructure devices.

interesting candidate for the substitution is the infinite-layer $n$-type cuprate $\operatorname{Sr}_{1-\mathrm{x}} \mathrm{Ln}_{\mathrm{x}} \mathrm{CuO}_{2}$ $(\mathrm{Ln}=\mathrm{La}, \mathrm{Gd}, \mathrm{Sm})$ superconductor, which reveals strong spectroscopic evidences for a pure $s$-wave pairing symmetry $[134,4]$. Such substitution can provide useful new information for several reasons. First of all, the $c$-axis superconducting coherence length in this material is longer than the $c$-axis lattice constant, thus, this system is expected to reveal more threedimensional characteristics. It would be interesting to study the effects of spin-injection on this less anisotropic system. Second, pure $s$-wave pairing symmetry is fully gapped, thus, the interactions and dynamic between the injected quasiparticles with the low-energy excitations in the gapless nodal directions may no longer be an issue. It is likely that the injection of spin-polarized quasiparticles would have different effects on superconductivity in the $n$-type cuprates with $s$-wave pairing because of a fully gapped Fermi surface that requires injection of these quasiparticles at an energy larger than the superconducting gap, and these energetic spin-polarized quasiparticles would have to undergo both energy and spin relaxation processes. 


\section{Bibliography}

[1] V. L. Ginzburg and L. Landau. Zh. Eksp. Teor. Fiz., 20:1064, 1950.

[2] J. Bardeen, L. N. Cooper, and J. R. Schrieffer. Phys. Rev., 108:1175, 1957.

[3] G. Bednorz and K. A. Müller. Z. Phys., B64:189, 1986.

[4] N.-C. Yeh. Recent advances in high-temperature superconductivity. Assoc. APPS Bulletin, in press, 2002.

[5] J. Orenstein and A. J. Millis. Science, 288:468, 2000.

[6] S. Sachdev. Science, 288:475, 2000.

[7] M. Vojta, Y. Zhang, and S. Sachdev. Phys. Rev. B, 62:6721, 2000.

[8] D. J. Van Harlingen. Rev. Mod. Phys., 67:515, 1995.

[9] C. C. Tsuei and J. R. Kirtley. Rev. Mod. Phys., 72:969, 2000.

[10] N.-C. Yeh, C.-T. Chen, G. Hammerl, J. Mannhart, A. Schmehl, C. W. Schneider, R. R. Schulz, S. Tajima, K. Yoshida, D. Garrigus, and M. Strasik. Phys. Rev. Lett., 87:087003, 2001. 
[11] N.-C. Yeh, C.-T. Chen, G. Hammerl, J. Mannhart, S. Tajima, K. Yoshida, A. Schmehl, C. W. Schneider, and R. R. Schulz. Physica C, 364-365:450, 2001.

[12] J. Y. T. Wei, N.-C. Yeh, D. F. Garrigus, and M. Strasik. Phys. Rev. Lett., 81:2542, 1998.

[13] J. Y. T. Wei et al. Physica B, 284:973, 2000.

[14] F. Tafuri and J. R. Kirtley. Phys. Rev. B, 62:13934, 2000.

[15] L. Alff, S. Meyer, S. Kleefisch, U. Schoop, A. Marx, H. Sato, M. Naito, and R. Gross. Phys. Rev. Lett., 83:2644, 1999.

[16] C. C. Tsuei and J. R. Kirtley. Phys. Rev. Lett., 85:182, 2000.

[17] J. A. Skinta, M.-S. Kim, and T. R. Lemberger. Phys. Rev. Lett., 88:207005, 2002.

[18] A. Biswas, P. Fournier, M. M. Qazilbash, V. N. Smolyaninova, H. Balci, and R. L. Greene. Phys. Rev. Lett., 88:207004, 2002.

[19] D. Pines. Physica C, 282-287:273, 1993.

[20] P. W. Anderson. Science, 235:1196, 1987.

[21] G. Kotliar. Phys. Rev. B, 37:3664, 1988.

[22] P. A. Lee and X. G. Wen. Phys. Rev. Lett., 78:4111, 1997.

[23] V. J. Emery, S. A. Kivelson, and O. Zachar. Phys. Rev. B, 56:6120, 1997.

[24] G. Siebold, C. Castellani, D. DiCastro, and M. Grilli. Phys. Rev. B, 58:13506, 1998. 
[25] C. M. Varma. Phys. Rev. B, 55:14554, 1997.

[26] A. V. Balatsky, M. I. Salkola, and A. Rosengren. Phys. Rev. B, 51:15547, 1995.

[27] M. E. Flatte and J. M. Byers. Phys. Rev. Lett., 80:4546, 1998.

[28] M. I. Salkola and J. R. Schrieffer. Phys. Rev. B, 58:R5952, 1998.

[29] S. Haas and K. Maki. Phys. Rev. Lett., 85:2172, 2000.

[30] A. Polkovnikov, S. Sachdev, and M. Vojta. Phys. Rev. Lett., 86:296, 2001.

[31] H. Alloul, P. Mendels, H. Casalta, J. F. Maruicco, and J. Arabski. Phys. Rev. Lett., 67:3140, 1991.

[32] T. Miyatake, K. Yamaguchi, T. Takata, N. Koshizuka, and S. Tanaka. Phys. Rev. B, 44:10139, 1991.

[33] G.-Q. Zheng, T. Odaguchi, Y. Kitaoka, K. Asayama, Y. Kodama, K. Mizuhashi, and S. Uchida. Physica C, 263:367, 1996.

[34] K. Ishida, Y. Kitaoka, K. Yamazoe, and K. Asayama. Phys. Rev. Lett., 76:531, 1996.

[35] H. F. Fong, P. Bourges, Y. Sidis, L. P. Regnault, J. Bossy, A. Ivanov, D. L. Millius, I. A. Aksay, and B. Keimer. Phys. Rev. Lett., 82:1939, 1999.

[36] K. Tomimoto, I. Terasaki, A. I. Rykov, T. Mimura, and S. Tajima. Phys. Rev. B, 60:114, 1999.

[37] N. L. Wang, S. Tajima, A. I. Rykov, and K. Tomimoto. Phys. Rev. B, 57:R11081, 1998. 
[38] S. H. Pan, E. W. Hudson, K. M. Lang, H. Eisaki, S. Uchida, and J. C. Davis. Nature, 403:746, 2000.

[39] J. Figueras, T. Puig, A. E. Carrillo, and X. Obradors. Supercond. Sci. Technol., $13: 1067,2000$.

[40] W. A. MacFarlane, J. Bobroff, H. Alloul, P. Mendels, N. Blanchard, G. Collin, and J.-F. Marucco. Phys. Rev. Lett., 85:1108, 2000.

[41] Y. Sidis, P. Bourges, H. F. Fong, B. Keimer, L. P. Regnault, J. Bossy, A. Ivanov, B. Hennion, P. Gautier-Picard, G. Collin, D. L. Millius, and I. A. Aksay. Phys. Rev. Lett., 84:5900, 2000.

[42] P. W. Anderson. J. Phys. Chem. Solids, 11:26, 1959.

[43] A. Yazdani, B. A. Jones, C. P. Lutz, M. F. Crommie, and D. M. Eigler. Science, 275:1767, 1997.

[44] Q. Si. Phys. Rev. Lett., 78:1767, 1997.

[45] M. Tinkham. Introduction to Superconductivity. McGraw Hill, New York, second edition edition, 1996. and references therein.

[46] A. Schmid and G. Schon. J. Low Temp. Phys., 20:207, 1975.

[47] A. A. Abrikosov and L. P. Gor'kov. Sov. Phys. JETP, 12:1243, 1961.

[48] For a collection of review articles, see . Nonequilibrium Superconductivity, Phonons, and Kapitza Boundaries, volume 65 of NATO Advanced Study Institute, Series B: Physics. Plenum, New York, 1981. edited by K. E. Gray. 
[49] P. M. Tedrow and R. Meservey. Phys. Rev. B, 7:318, 1973.

[50] G. A. Prinz. Spin-polarized transport. Physics Today, 48(4):85, 1995. and references therein.

[51] V. A. Vas'ko, V. A. Larkin, P. A. Kraus, K. R. Nikolaev, D. E. Grupp, C. A. Nordman, and A. M. Goldman. Phys. Rev. Lett., 78:1134, 1997.

[52] Z. W. Dong, R. Ramesh, T. Venkatesan, M. Johnson, Z. Y. Chen, S. P. Pai, V. Talyansky, R. P.Sharma, R. Sheekala, C. J. Lobb, and R. L. Greene. Appl. Phys. Lett., $71: 1718,1997$.

[53] N.-C. Yeh, R. P. Vasquez, C.-C. Fu, A. V. Samoilov, Y. Li, and K. Vakili. Phys. Rev. $B, 60: 10522,1999$.

[54] N.-C. Yeh, J. Y. T. Wei, C.-C. Fu, and R. P. Vasquez. Physica B, 284-288:507, 2000.

[55] J. Y. T. Wei, N.-C. Yeh, and R. P. Vasquez. J. Appl. Phys., 85:5350, 1999.

[56] W. E. Pickett and D. J. Singh. Phys. Rev. B, 53:1146, 1996.

[57] H. Y. Hwang, S. W. Cheong, P. G. Radaelli, M. Marezio, and B. Battlog. Phys. Rev. Lett., 75:914, 1995.

[58] N.-C. Yeh, R. P. Vasquez, D. A. Beam, C.-C. Fu, J. Huynh, and G. Beach. J. Phys.: Condens. Matter, 9:3713, 1997.

[59] N.-C. Yeh, C.-C. Fu, J. Y. T. Wei, R. P. Vasquez, J. Huynh, S. M. Maurer, D. A. Beam, and G. Beach. J. Appl. Phys., 81:5499, 1997. 
[60] J. Y. T. Wei, N.-C. Yeh, and R. P. Vasquez. Phys. Rev. Lett., 79:5150, 1997.

[61] V. A. Vas'ko, K. R. Nikolaev, V. A. Larkin, P. A. Kraus, and A. M. Goldman. Appl. Phys. Lett., 73:844, 1998.

[62] N.-C. Yeh, J. Y. T. Wei, C.-T. Chen, W. D. Si, and X. X. Xi. Physica C, 341-348:1639, 2000.

[63] S. Bhattacharjee and M. Sardar. Phys. Rev. B, 62:R6139, 2000.

[64] B. J. Feenstra, J. Schutzmann, D. van der Marel, R. Perez Pinaya, and M. Decroux. Phys. Rev. Lett., 79:4890, 1997.

[65] J. Clarke and M. Tinkham. Phys. Rev. Lett., 28:1363, 1972.

[66] M. Tinkham and J. Clarke. Phys. Rev. Lett., 28:1366, 1972.

[67] M. Tinkham. Phys. Rev. B, 6:1747, 1972.

[68] J. Clarke and J. L. Paterson. J. Low Temp. Phys., 15:491, 1974.

[69] J. Clarke, U. Eckern, A. Schmid, G. Schon, and M. Tinkham. Phys. Rev. B, 20:3933, 1979.

[70] A. F. G. Wyatt, V. M. Dmitriev, W. S. Moore, and F. W. Sheard. Phys. Rev. Lett., $16: 1166,1966$.

[71] A. H. Dayem and J. J. Wiegand. Phys. Rev., 155:419, 1967.

[72] T. M. Klapwijk and J. E. Mooij. Physica, 81B:132, 1976. 
[73] T. M. Klapwijk, J. N. van den Bergh, and J. E. Mooij. J. Low Temp. Phys., 26:385, 1977.

[74] J. E. Mooij, N. Lambert, and T. M. Klapwijk. Solid State Comm., 36:585, 1980.

[75] G. M. Eliashberg. JETP Lett., 11:114, 1970.

[76] G. M. Eliashberg. Sov. Phys. JETP, 34:668, 1972.

[77] A. Schmid. Phys. Rev. Lett., 38:922, 1977.

[78] L. G. Aslamazov and A. I. Larkin. Sov. Phys. JETP, 44:178, 1976.

[79] S. B. Kaplan, C. C. Chi, D. N. Langenberg, J. J. Chang, S. Jaferey, and D. J. Scalapino. Phys. Rev. B, 14:4854, 1976.

[80] T. Kommers and J. Clarke. Phys. Rev. Lett., 38:1091, 1977.

[81] J. J. Chang and D. J. Scalapino. J. Low Temp. Phys., 31:1, 1978.

[82] P. C. van Son, J. Romijn, T. M. Klapwijk, and J. E. Mooij. Phys. Rev. B, 29:1503, 1984.

[83] C. C. Chi and J. Clarke. Phys. Rev. B, 20:4465, 1979.

[84] R. P. Vasquez, M. C. Foote, L. Bajuk, and B. D. Hunt. J. Elec. Spec., 57:317, 1991.

[85] D. S. Reed. Frequency Dependent Investigation of the Vortex Dynamics in High Temperature Superconductors. Caltech, Pasadena, 1995.

[86] R. von Helmolt, J. Wecker, B. Holzapfel, L. Schultz, and K. Samwer. Phys. Rev. Lett., 71:2331, 1993. 
[87] S. Jin, T. H. Tiefel, M. McCormack, R. A. Fastnacht, R. Ramesh, and L. H. Chen. Science, 264:413, 1994.

[88] A. J. Millis, P. B. Littlewood, and B. I. Shraiman. Phys. Rev. Lett., 74:5144, 1995.

[89] S. Jin, H. M. O’Bryan, T. H. Tiefel, M. McCormack, and W. W. Rhodes. Appl. Phys. Lett., 66:382, 1995 .

[90] K. Khazeni, Y. X. Jia, L. Lu, V. H. Crespi, M. L. Cohen, and A. Zettl. Phys. Rev. Lett., 76:295, 1996.

[91] G. H. Jonker and J. H. van Santen. Physica, 16:337, 1950.

[92] C. Zener. Phys. Rev., 82:403, 1951.

[93] E. O. Wollan and W. C. Koehler. Phys. Rev., 100:545, 1955.

[94] J. B. Goodenough, A. Wold, R. J. Arnott, and N. Menyuk. J. Appl. Phys., 124:373, 1961.

[95] P. G. de Gennes. Phys. Rev., 118:141, 1960.

[96] A. J. Millis, B. I. Shraiman, and R. Mueller. Phys. Rev. Lett., 77:175, 1996.

[97] S. Jin, T. H. Tiefel, M. McCormack, H. M. O’Bryan, L. H. Chen, R. Ramesh, and D. Schurig. Appl. Phys. Lett., 67:557, 1995.

[98] Y. X. Jia, L. Lu, K. Khazeni, V. H. Crespi, A. Zettl, and M. L. Cohen. Phys. Rev. $B, 52: 9147,1995$. 
[99] M. R. Ibarra, P. A. Algarabel, C. Marquina, J. Blasco, and J. Garcia. Phys. Rev. Lett., 75:3541, 1995.

[100] G.-M. Zhao, K. Conder, H. Keller, and K. A. Muller. Nature, 381:676, 1996.

[101] H. Ohbayashi, T. Kudo, and T. Gejo. Japan. J. Appl. Phys., 13:1, 1974.

[102] R. Mahendiran, A. K. Raychaudhuri, A. Chainani, and D. D. Sarma. J. Phys.: Condens. Matter, 7:L561, 1995.

[103] S. Yamaguchi, H. Taniguchi, H. Takagi, T. Arima, and Y. Tokura. J. Phys. Soc. Japan, 64:1885, 1995.

[104] R. P. Vasquez. Phys. Rev. B, 54:14938, 1996.

[105] N.-C. Yeh, R. P. Vasquez, J. Y. T. Wei, C.-C. Fu, G. Beach, J. Huynh, A. V. Samoilov, A. V. Boris, N. N. Kovaleva, and A. V. Bazhenov. Epitaxial Oxide Thin Films- III, invited paper, Mat. Res. Soc. Proc., 1997.

[106] A. V. Boris, A. V. Bazhenov, N. N. Kovaleva, A. V. Samoilov, N.-C. Yeh, and R. P. Vasquez. J. Appl. Phys., 81:5756, 1997.

[107] K. H. Kim, J. Y. Gu, H. S. Choi, G. W. Park, and T. W. Noh. Phys. Rev. Lett., $77: 1877,1996$.

[108] N. F. Mott and E. A. Davis. Electronic Processes in Non-crystalline Materials. Oxford, Clarendon, 1979.

[109] R. M. Kusters, J. Singleton, D. A. Keen, R. McGreevy, and W. Hayes. Physica B, $155: 362,1989$. 
[110] M. Fath, S. Freisem, A. A. Menovsky, Y. Tomioka, J. Aarts, and J. A. Mydosh. Science, 285:1540, 1999.

[111] E. Dagotto, T. Hotta, and A. Moreo. Phys. Rep., 344:1, 2001.

[112] A. Moreo, M. Mayr, A. Feiguin, S. Yunoki, and E. Dagotto. Phys. Rev. Lett., 84:5568, 2000.

[113] A. V. Samoilov, G. Beach, C.-C. Fu, N.-C. Yeh, and R. P. Vasquez. J. Appl. Phys., 83:6998, 1998.

[114] N.-C. Yeh, C.-C. Fu, R. P. Vasquez, and A. V. Samoilov. Physica B, 284:1446, 2000.

[115] A. V. Boris, N. N. Kovaleva, A. V. Bazhenov, P. J. M. van Bentum, T. Rasing, S. W. Cheong, A. V. Samoilov, and N.-C. Yeh. Phys. Rev. B, 59:R697, 1999.

[116] C.-C. Fu, Z. Huang, and N.-C. Yeh. Phys. Rev. B, 65:224516, 2002.

[117] P. Mikheenko, R. Chakalov, C. Severac, F. Wellhofer, M. Colclough, C. Muirhead, and K. Kawano. Physica C, 341:2711, 2000.

[118] H. Montgomery and G. P. Pells. Br. J. Appl. Phys., 14:525, 1963.

[119] J. X. Zhu, B. Friedman, and C. S. Ting. Phys. Rev. B, 59:9558, 1999.

[120] P. G. de Gennes. Superconductivity in Metals and Alloys. Addison-Wesley, Reading, MA, 1989. and references therein.

[121] R. S. Markiewicz. J. Phys. Chem. Solids, 58:1179, 1997.

[122] N. J. Curro, T. Imai, C. P. Slichter, and B. Dabrowski. Phys. Rev. B, 56:877, 1997. 
[123] G. Blatter, M. V. Feigel'man, V. B. Geshkenbein, A. I. Larkin, and V. M. Vinokur. Rev. Mod. Phys., 66:1125, 1994.

[124] K. Maki. Gapless Superconductivity, volume II. Dekker, New York, 1969. in Superconductivity, Vol. II, R. D. Parks (ed.).

[125] Y. Gim, A. W. Kleinsasser, and J. B. Barner. J. Appl. Phys., 90:4063, 2001.

[126] Y. M. Boguslavskij, K. Joosse, F. J. G. Roesthuis, G. J. Gerritsma, and H. Rogalla. Physica B, 194-196:85, 1994.

[127] C. W. Schneider, R. Moerman, F. J. G. Roesthuis, G. J. Gerritsma, and H. Rogalla. Inst. Phys. Conf. Ser., 148:1677, 1995.

[128] P. Mikheenko, M. S. Colclough, C. Severac, R. Chakalov, F. Welhoffer, and C. M. Muirhead. Appl. Phys. Lett., 78:356, 2001.

[129] R. Glang. Handbook of Thin Film Technology. McGraw Hill, New York, 1970.

[130] L. Holland. Vacuum Deposition of Thin Films. Chapman and Hall, London, 1957.

[131] J. L. Vossen and W. Kern. Thin Film Processes II. Academic Press, Inc., Boston, 1991.

[132] H. Murrmann and D. Widmann. IEEE Trans. Electron. Devices, ED-16:1022, 1969.

[133] H. Murrmann and D. Widmann. Solid-St. Electron., 12:879, 1969.

[134] C.-T. Chen, P. Seneor, N.-C. Yeh, R. P. Vasquez, L. D. Bell, C. U. Jung, J. Y. Kim, M.-S. Park, H.-J. Kim, and S.-I. Lee. Phys. Rev. Lett., 88:227002, 2002. 